\title{
Separation of variables in the WZW models
}

\author{
Oleg Lunin ${ }^{a}$ and Jia $\operatorname{Tian}^{b}$ \\ ${ }^{a}$ Department of Physics, University at Albany (SUNY), \\ 1400 Washington Av., Albany, NY 12222, U.S.A. \\ ${ }^{b}$ Kavli Institute for Theoretical Sciences (KITS), University of Chinese Academy of Science, \\ No. 3, Nanyitiao, Zhongguancun, Haidian District, Beïing 100190, P.R. China \\ E-mail: olunin@albany.edu, wukongjiaozi@ucas.ac.cn
}

\begin{abstract}
We consider dynamics of scalar and vector fields on gravitational backgrounds of the Wess-Zumino-Witten models. For $\mathrm{SO}(4)$ and its cosets, we demonstrate full separation of variables for all fields and find a close analogy with a similar separation of vector equations in the backgrounds of the Myers-Perry black holes. For $\mathrm{SO}(5)$ and higher groups separation of variables is found only in some subsectors.
\end{abstract}

Keywords: Conformal Field Models in String Theory, Space-Time Symmetries

ARXiv EPrint: 2012.15083 


\section{Contents}

1 Introduction 1

2 Full separation in $\mathrm{SO}(4)$ and its cosets 3

2.1 Wzw model for $\mathrm{SO}(4)$

$\begin{array}{lll}2.2 & \text { Vector fields on the } \mathrm{SO}(4) \text { wZW model } & 7\end{array}$

$\begin{array}{lll}2.2 .1 & \text { Vector fields on product spaces } & 7\end{array}$

2.2.2 Vector modes on $\mathrm{SU}(2) \quad 9$

$\begin{array}{ll}\text { 2.2.3 Summary of the vector fields on } \mathrm{SO}(4) & 13\end{array}$

2.3 Gauged WZW model for the $\mathrm{SO}(4) / \mathrm{SO}(2)$ coset 15

2.4 Vector fields on the $\mathrm{SO}(4) / \mathrm{SO}(2)$ gauged WZW model 18

2.5 Scalars and vectors on the $\frac{\mathrm{SO}(4)}{\mathrm{SO}(2) \times \mathrm{SO}(2)}$ gauged WZW model 20

$\begin{array}{lll}2.6 & \text { Gauging and T-duality } & 25\end{array}$

3 The SO(5) sigma model $\quad 27$

$\begin{array}{ll}3.1 & \text { Eigenfunctions from group theory } \\ 3.2 & 28\end{array}$

$\begin{array}{lll}3.2 & \text { Factorization of the } R \text { dependence } & 31\end{array}$

3.3 Separable $X$-independent solutions $\quad 32$

3.4 Solutions linear in $X$ coordinates 34

$\begin{array}{ll}3.5 & \text { Separable states in symmetric representations } 38\end{array}$

3.6 Separation in terms of spherical harmonics 41

4 Discussion $\quad 43$

A Vector field on the SU(2) WZW model 43

A.1 Standard equation for the vector: $\zeta=0 \quad 44$

A.2 Modified vector equation: $\operatorname{arbitrary} \zeta \quad 46$

B Geometries for the gauged WZW models 49

C Scalar wavefunctions for the $\mathrm{SO}(5)$ sigma model $\quad 50$

$\begin{array}{ll}\text { C.1 Solutions with linear } z \text {-dependence } & 50\end{array}$

C.2 Wavefunctions in the symmetric representations 51

\section{Introduction}

Exact solutions of string theory and supergravity provide indispensable insights into dynamics of strongly coupled systems. Once the relevant gravitational backgrounds are found, one extract their physical properties by studying excitations of various fields of these geometries. Unfortunately this problem, which often involves study of partial differential 
equations (PDEs), is very difficult unless the geometry has a high degree of symmetry. In such cases one can solve dynamical equations for various fields using separation of variables, and applications of this technique range from simple geometries, such as flat space and spheres, to spacetimes with relatively few isometries, such as rotating black holes in various dimensions. In this article we study separation of variables in the (gauged) WessZumino-Witten (WZW) models [1], the spaces that have either very few isometries or none at all, and show that in certain cases the variables in the scalar and vector equations can be separated. This success is the consequence of the algebraic symmetries of the WZW models, which are guaranteed by the CFT construction, but which are not obvious from the target space perspective.

The dynamics of a scalar field has been studied in the WZW models and their gauged version, and the full spectrum of eigenvalues is known $[2,3] .^{1}$ The construction of the relevant wavefunctions is a more complicated problem, and it has been solved only on a case-by-case basis $[2,3,5]$. The techniques used in $[2,3]$ and [5] were purely algebraic, and they did not rely on separation of variables, which is not expected to happen for most (gauged) WZW backgrounds. Unfortunately there are no known procedures for extending these algebraic techniques to vector and tensor fields, especially for finding the eigenfunctions. ${ }^{2}$ On the other hand, experience with other backgrounds, such as black hole geometries, shows that separation of variables for a scalar field [7-28] is often accompanied by separation in the vector and tensor equations [29-36]. Inspired by this success, we focus on the gWZW models which admit separation of variables in the Helmholtz equation for a scalar and demonstrate that separability of the vector equation in all such cases. We also analyze an example of the WZW model that admits separation of variables only in some subsectors, and we find the explicit expressions for the resulting solutions.

Wess-Zumino-Witten models are exactly solvable conformal field theories [1], and their numerous applications range from black holes $[4,37]$ to the quantum Hall effect [38]. In the last few years these models have been used to construct families of integrable string theories describing deformations of the systems appearing in the AdS/CFT correspondence [39-54], and in this context the deformations of the $\mathrm{SO}(n)$ and $\mathrm{SO}(n) / \mathrm{SO}(n-1)$ WZW models are particularly important. Given this interest, we will focus on studying such models with $n=3,4,5$. The eigenvalues and eigenvectors of the scalar field on such background are known through the algebraic constructions $[2,3,5],{ }^{3}$ but it is not clear whether these algebraic methods can be extended to the vector field. By performing an additional analysis of the solutions discussed in [5], we demonstrate an existence of a coordinate system where scalar equations fully separate for the (gauged) WZW models on $\mathrm{SO}(4)$ and its cosets, and we identify the Killing-Yano tensors [55-57] associated with this separation. We then study the vector equations on such backgrounds and find the unique way to separate them. Note that separability of vector equations is more subtle than in the scalar case since knowing the correct coordinate system is not sufficient. One has to identify the correct modes of the

\footnotetext{
${ }^{1}$ Similar ideas were also explored earlier in [4].

${ }^{2}$ Application of algebraic techniques for computing vector eigenvalues will be discussed in [6].

${ }^{3}$ Article [5] also solved the scalar equation on the $\lambda$-deformed backgrounds [39-42], but we will not discuss such deformations in this paper.
} 
vector field as well. In the Myers-Perry geometry of rotating black holes [58] this problem was solved in [31], where it was shown that the separable components of the gauge field are obtained by taking projections to the frames associated with the Killing-Yano tensor. In this article we prove that the same statement holds for the $\mathrm{SO}(4) / H$ WZW models as well, and separation of variables in these cases is very similar to the one found in [31].

Unfortunately the full separation of variable does not seem to persist for the $\mathrm{SO}(n)$ WZW model with $n>4$. Nevertheless in the $\mathrm{SO}(5)$ several 4-parametric families of separable solutions which have nontrivial dependences on all 10 coordinates. These wavefunctions are found by combining algebraic techniques with analysis of PDEs.

This paper has the following organization. In section 2 we consider the WZW model for the $\mathrm{SO}(4)$ group and demonstrate full separability of dynamical equations for a scalar and a vector. While these results are expected, at least for the scalar, since $\mathrm{SO}(4)=$ $\mathrm{SU}(2) \times \mathrm{SU}(2)$, and a metric on each $\mathrm{SU}(2)$ has only one non-cyclic direction, the detailed analysis of separability for the vector field reveals interesting structures which can be extended to the situations where separation of variables is less obvious. We do this in sections 2.3, 2.4, and 2.5, which focus on the gauged WZW models for various cosets of $\mathrm{SO}(4)$. In section 2.6 we show how separable structures on such spaces are mapped into each other under $\mathrm{T}$ duality.

Section 3 is dedicated to the study of a scalar field on the $\mathrm{SO}(5)$ group manifold. While the equations are not fully separable, we identify several important sectors that admit partial separation. Interestingly, all these situations lead to infinite families of solutions which depend on four free parameters. At least one of these families has a simple extension to all $\mathrm{SO}(N)$ groups, which is discussed in section 3.6. The results presented in section 3 are obtained by combining the analysis of the differential equation for the scalar field (sections 3.2, 3.4, 3.3, 3.6) and a pure algebraic construction of the eigenfunctions developed in sections 3.1 and 3.5. Some technical details are presented in the appendices.

\section{Full separation in $\mathrm{SO}(4)$ and its cosets}

The goal of this article is to explore separation of variables for various excitations of the WZW models. In this section we begin with the $\mathrm{SO}(4)$ case, where such separation is obvious in the equation for the scalar field. Then we analyze the equations for the vector field and identify the components that separate as well. In sections $2.3-2.5$ we demonstrate that equations for the scalar and vector fields remain separable even after some subgroups of $\mathrm{SO}(4)$ are gauged. We show that all these situations follow the pattern discovered in the case of higher dimensional rotating black holes: the separable components of the vector field are constructed by taking projections to the frames associated with the Killing-Yano tensors [31]. Our analysis serves as a derivation of the separable ansatz for $\mathrm{SO}(4)$ and its cosets since we prove that no other components of the vector fields are separable and that the correct number of polarizations is recovered. In this section we focus only on cosets taken with respect to abelian subgroups, but full separation in the non-abelian cases, such as $\mathrm{SO}(4) / \mathrm{SU}(2)$ and $\mathrm{SU}(2)_{k_{1}} \times \mathrm{SU}(2)_{k_{2}} / \mathrm{SU}(2)_{k_{1}+k_{2}}$, is unlikely. Scalar excitations on these 
spaces were studied in $[2,3]$ using algebraic techniques, and it would be interesting to extend this analysis to vector fields.

\subsection{Wzw model for $\mathrm{SO}(4)$}

We begin with studying excitations of the $\mathrm{SO}(4)$ group manifold. The action of the WZW model is given by [1]

$$
S=-\frac{k}{2 \pi} \int d^{2} \sigma \eta^{\alpha \beta} \operatorname{tr}\left(g^{-1} \partial_{\alpha} g g^{-1} \partial_{\beta} g\right)+\frac{i k}{6 \pi} \int \operatorname{tr}\left(g^{-1} d g \wedge g^{-1} d g \wedge g^{-1} d g\right),
$$

where $g$ is an element of $\mathrm{SO}(4)$. Since the WZW background is conformal, the dilaton is trivial. ${ }^{4}$ We consider various excitations of the background (2.1), such as scalar and vector fields propagating on the geometry with a metric

$$
d s^{2}=-\frac{k}{2 \pi} \operatorname{tr}\left(g^{-1} d g g^{-1} d g\right) .
$$

Bearing in mind extensions to larger groups discussed in the next section, we parameterize an element of $\mathrm{SO}(4)$ as $^{5}$

$$
g=\left[\begin{array}{cc}
q_{2}\left(\alpha_{L}\right) & 0 \\
0 & q_{2}\left(\beta_{L}\right)
\end{array}\right]\left[\begin{array}{cc}
I-\frac{2}{1+X X^{T}} X X^{T} & \frac{2}{1+X X^{T}} X \\
-X^{T} \frac{2}{1+X X^{T}} & I-\frac{2}{1+X^{T} X} X^{T} X
\end{array}\right]\left[\begin{array}{cc}
q_{2}\left(\alpha_{R}\right) & 0 \\
0 & q_{2}\left(\beta_{R}\right)
\end{array}\right] .
$$

Here $X=\operatorname{diag}\left(X_{1}, X_{2}\right)$ is a diagonal $2 \times 2$ matrix, and $q_{2}(\gamma)$ are elements of $\mathrm{SO}(2)$ :

$$
q_{2}(\gamma)=\left[\begin{array}{cc}
\cos \gamma & \sin \gamma \\
-\sin \gamma & \cos \gamma
\end{array}\right]
$$

To justify the parameterization (2.3), we observe that the action (2.1) is invariant under $F=\mathrm{SO}(4) \times \mathrm{SO}(4)$ transformations, $g \rightarrow h_{L} g h_{R}$. To separate variables in various dynamical equations, it is convenient to maximize the number of cyclic directions in the metric (2.2). Such cyclic directions correspond to commuting U(1) subgroups of $F$, and there are at most four of them since $F$ has rank four. Therefore, it is convenient to choose a parameterization where the $[\mathrm{U}(1)]^{4}$ Cartan subgroup of $F$ is realized by simple shifts of coordinates $\left(\alpha_{L}, \beta_{L}, \alpha_{R}, \beta_{R}\right)$, and this is accomplished by the introduction of the left and the right matrices in (2.3). In sections 2.3 and 2.5 some elements of the Cartan group $[\mathrm{U}(1)]^{4}$ will be gauged by setting some of the four angular coordinates to zero. The matrix in the middle of (2.3) contains the remaining two out of six parameters of $\mathrm{SO}(4)$. Although one can start with an arbitrary $2 \times 2$ matrix $X$ there, the transformation $X \rightarrow q_{2}\left(\gamma_{L}\right) g q_{2}\left(\gamma_{R}\right)$ can be used to diagonalize that matrix, and parameters $\left(\gamma_{L}, \gamma_{R}\right)$ can be removed by shifting the Cartan coordinates. This leads to the parameterization (2.3) which ensures that the metric (2.2) has four cyclic directions corresponding to the Cartan subgroup of $\mathrm{SO}(4) \times \mathrm{SO}(4)$, and this is the maximal number of the cyclic directions for the $\mathrm{SO}(4) \mathrm{WZW}$ model.

\footnotetext{
${ }^{4}$ Gauging of some symmetries leads to a non-trivial dilaton [59]. We will discuss this in more detail below.

${ }^{5}$ Similar parameterization for other groups and cosets was introduced in [60].
} 
Substituting the parameterization (2.3) into (2.2), we arrive at the metric

$$
\begin{aligned}
d s^{2}= & \frac{4 k}{\pi}\left[\frac{d X_{1}^{2}}{\left(X_{1}^{2}+1\right)^{2}}+\frac{d X_{2}^{2}}{\left(X_{2}^{2}+1\right)^{2}}\right]+\frac{k}{\pi}\left[d \alpha_{L}^{2}+d \beta_{L}^{2}+d \alpha_{R}^{2}+d \beta_{R}^{2}\right] \\
& +\frac{k}{\pi}\left[\frac{8 X_{1} X_{2}\left(d \alpha_{L} d \beta_{R}+d \alpha_{R} d \beta_{L}\right)}{\left(1+X_{1}^{2}\right)\left(1+X_{2}^{2}\right)}+\frac{2\left(1-X_{1}^{2}\right)\left(1-X_{2}^{2}\right)}{\left(1+X_{1}^{2}\right)\left(1+X_{2}^{2}\right)}\left(d \alpha_{L} d \alpha_{R}+d \beta_{L} d \beta_{R}\right)\right] .
\end{aligned}
$$

The Kalb-Ramond field is given by

$$
\begin{aligned}
B= & \frac{k}{\pi} \frac{\left(X_{1}-X_{2}\right)^{2}}{\left(X_{1}^{2}+1\right)\left(X_{2}^{2}+1\right)}\left(d \alpha_{L}+d \beta_{L}\right) \wedge\left(d \alpha_{R}+d \beta_{R}\right) \\
& \frac{k}{\pi} \frac{\left(X_{1}+X_{2}\right)^{2}}{\left(X_{1}^{2}+1\right)\left(X_{2}^{2}+1\right)}\left(d \alpha_{L}-d \beta_{L}\right) \wedge\left(d \alpha_{R}-d \beta_{R}\right) .
\end{aligned}
$$

As expected, this geometry has four cyclic coordinates $\left(\alpha_{L}, \beta_{L}, \alpha_{R}, \beta_{R}\right)$, so solutions of the Helmholtz equation

$$
\nabla^{2} \Phi=-\Lambda \Phi
$$

can be written in the form

$$
\Phi=e^{i n_{1} \alpha_{L}+i n_{2} \beta_{L}+i n_{3} \alpha_{R}+i n_{4} \beta_{R}} \tilde{\Phi}\left(X_{1}, X_{2}\right) .
$$

A direct inspection of the metric (2.5) and its inverse shows that variables $\left(X_{1}, X_{2}\right)$ do not separate in the equation (2.7). On the other hand, since $\mathrm{SO}(4)=\mathrm{SU}(2)_{1} \times \mathrm{SU}(2)_{2}$, there is an alternative parameterization of the group element where the full separation is guaranteed. Specifically, writing an element of $\mathrm{SU}(2)_{1}$ as

$$
g_{1}=\left[\begin{array}{cc}
e^{i \gamma_{1}} & 0 \\
0 & e^{-i \gamma_{1}}
\end{array}\right]\left[\begin{array}{cc}
\cos \mu_{1} & \sin \mu_{1} \\
-\sin \mu_{1} & \cos \mu_{1}
\end{array}\right]\left[\begin{array}{cc}
e^{i \tau_{1}} & 0 \\
0 & e^{-i \tau_{1}}
\end{array}\right]
$$

and using a similar expression for $g_{2}$, we find

$$
\begin{aligned}
d s^{2} & =\frac{k}{\pi} \sum_{j=1}^{2}\left[d \mu_{j}^{2}+\left(d \gamma_{j}+d \tau_{j}\right)^{2}-4 \sin ^{2} \mu_{j} d \gamma_{j} d \tau_{j}\right] \\
B & =\frac{k}{\pi} \sum_{j=1}^{2} \cos \left(2 \mu_{j}\right) d \gamma_{j} \wedge d \tau_{j} .
\end{aligned}
$$

Comparison of the $B$-field with (2.6) suggests the system of separable coordinates:

$$
y_{1}=1-\frac{2\left(X_{1}+X_{2}\right)^{2}}{\left(X_{1}^{2}+1\right)\left(X_{2}^{2}+1\right)}, \quad y_{2}=1-\frac{2\left(X_{1}-X_{2}\right)^{2}}{\left(X_{1}^{2}+1\right)\left(X_{2}^{2}+1\right)} .
$$

In the coordinates (2.11) the geometry (2.5)-(2.6) becomes

$$
\begin{aligned}
d s^{2}= & \frac{k}{2 \pi}\left[\frac{d y_{1}^{2}}{1-y_{1}^{2}}+\left(d \alpha_{L}-d \beta_{L}\right)^{2}+\left(d \alpha_{R}-d \beta_{R}\right)^{2}+2 y_{1}\left(d \alpha_{L}-d \beta_{L}\right)\left(d \alpha_{R}-d \beta_{R}\right)\right] \\
& +\frac{k}{2 \pi}\left[\frac{d y_{2}^{2}}{1-y_{2}^{2}}+\left(d \alpha_{L}+d \beta_{L}\right)^{2}+\left(d \alpha_{R}+d \beta_{R}\right)^{2}+2 y_{2}\left(d \alpha_{L}+d \beta_{L}\right)\left(d \alpha_{R}+d \beta_{R}\right)\right] \\
B= & \frac{k}{2 \pi}\left[\left(1-y_{1}\right)\left(d \alpha_{L}-d \beta_{L}\right) \wedge\left(d \alpha_{R}-d \beta_{R}\right)+\left(1-y_{2}\right)\left(d \alpha_{L}+d \beta_{L}\right) \wedge\left(d \alpha_{R}+d \beta_{R}\right)\right] .
\end{aligned}
$$


Imposing a separable ansatz for the scalar field,

$$
\Phi=e^{i n_{1}\left(\alpha_{L}-\beta_{L}\right)+i n_{2}\left(\alpha_{R}-\beta_{R}\right)+i \tilde{n}_{1}\left(\alpha_{L}+\beta_{L}\right)+i \tilde{n}_{2}\left(\alpha_{R}+\beta_{R}\right)} B_{1}\left(y_{1}\right) B_{2}\left(y_{2}\right),
$$

we arrive at the system of ODEs governing functions $\left(B_{1}, B_{2}\right)$ :

$$
\begin{aligned}
& \frac{d}{d y_{1}}\left[\left(y_{1}^{2}-1\right) B_{1}^{\prime}\right]+\left[-\nu_{1}+\frac{\left(n_{1}+n_{2}\right)^{2}}{2\left(1+y_{1}\right)}+\frac{\left(n_{1}-n_{2}\right)^{2}}{2\left(1-y_{1}\right)}\right] B_{1}=0 \\
& \frac{d}{d y_{2}}\left[\left(y_{2}^{2}-1\right) B_{2}^{\prime}\right]+\left[-\nu_{2}+\frac{\left(\tilde{n}_{1}+\tilde{n}_{2}\right)^{2}}{2\left(1+y_{2}\right)}+\frac{\left(\tilde{n}_{1}-\tilde{n}_{2}\right)^{2}}{2\left(1-y_{2}\right)}\right] B_{2}=0 .
\end{aligned}
$$

The eigenvalue of the Helmholtz equation (2.7) is given by

$$
\Lambda=\frac{2 \pi}{k}\left[\nu_{1}+\nu_{2}\right]
$$

The normalizable solution of the first equation in (2.14) is

$$
B_{1}\left(y_{1}\right)=\left(1+y_{1}\right)^{\frac{n_{+}}{2}}\left(1-y_{1}\right)^{\frac{n_{-}}{2}} F\left[-p, 1+p+n_{+}+n_{-} ; 1+n_{-} ; \frac{1-y_{1}}{2}\right],
$$

where $p$ is a non-negative integer and

$$
n_{ \pm}=\left|n_{1} \pm n_{2}\right|, \quad \nu_{1}=\frac{1}{4}\left(2 p+1+n_{+}+n_{-}\right)^{2}-\frac{1}{4} .
$$

Rewriting the last relation in a suggestive form

$$
\nu_{1}=j_{1}\left(j_{1}+1\right), \quad j_{1}=p+\frac{n_{+}+n_{-}}{2}
$$

we conclude that the eigenvalue $\nu_{1}$ is equal to the Casimir parameter for a representation of $\mathrm{SU}(2)$ described by a Young tableau with $2 j$ boxes. There is a similar expression for $\nu_{2}$,

$$
\nu_{2}=j_{2}\left(j_{2}+1\right), \quad j_{2}=\tilde{p}+\frac{\tilde{n}_{+}+\tilde{n}_{-}}{2}
$$

and single-valuedness of (2.13) as a function of $\left(\alpha_{L}, \beta_{L}, \alpha_{R}, \beta_{R}\right)$ implies that $\left(j_{1}, j_{2}\right)$ must be either integers or half-integers. The equation (2.15) gives the expression for the eigenvalue $\Lambda$ in terms of the Casimir of $\mathrm{SO}(4)$

$$
\Lambda=\frac{2 \pi}{k} C_{2}(R)
$$

This agrees with the general expression for the eigenvalues of scalars on the WZW backgrounds $[2,3]$. In the next subsection will extend these results to the vector field, and sections 2.3, 2.5 will focus on extensions of (2.13) and (2.20) to various cosets. 


\subsection{Vector fields on the $\mathrm{SO}(4)$ wZW model}

We have demonstrated separation of variables in the Helmholtz equation, and the next three subsections we will show that such separation persists for the analogous equation for the vector field: ${ }^{6}$

$$
e^{2 \sigma \phi} \nabla_{\mu}\left[e^{-2 \sigma \phi} \mathcal{F}^{\mu \nu}\right]+\Lambda A^{\nu}=0
$$

Here following [25-27], we introduced a modified field strength in the presence of torsion:

$$
\mathcal{F}_{\mu \nu}=\partial_{\mu} A_{\nu}-\partial_{\nu} A_{\mu}+\zeta H_{\mu \nu \sigma} A^{\sigma}=F_{\mu \nu}+\zeta H_{\mu \nu \sigma} A^{\sigma} .
$$

Let us consider equations (2.21)-(2.22) on $\mathrm{SO}(4)$ and its cosets.

\subsubsection{Vector fields on product spaces}

Since $\mathrm{SO}(4)=\mathrm{SU}(2) \times \mathrm{SU}(2)$ and $\mathrm{SO}(4) /[\mathrm{SO}(2) \times \mathrm{SO}(2)]=[\mathrm{SU}(2) / \mathrm{U}(1)]^{2}$ correspond to product spaces, we begin with a general discussion on vector fields on such manifolds. Specifically, we consider a geometry that has the form

$$
\begin{aligned}
d s^{2} & =g_{i j} d x^{i} d x^{j}+h_{a b} d y^{a} d y^{b}, \quad e^{-2 \phi}=f(x) \tilde{f}(y), \\
H & =\frac{1}{6} H_{i j k} d x^{i} \wedge d x^{j} \wedge d x^{k}+\frac{1}{6} H_{a b c} d y^{a} \wedge d y^{b} \wedge d y^{c} .
\end{aligned}
$$

Equations (2.21)-(2.22) on such a space become

$$
\begin{aligned}
& \frac{1}{f^{\sigma} \sqrt{g}} \partial_{i}\left[f^{\sigma} \sqrt{g} \mathcal{F}^{i j}\right]+\frac{1}{\tilde{f}^{\sigma} \sqrt{h}} \partial_{a}\left[\tilde{f}^{\sigma} \sqrt{h} F^{a j}\right]+\Lambda A^{j}=0 \\
& \frac{1}{f^{\sigma} \sqrt{g}} \partial_{i}\left[f^{\sigma} \sqrt{g} F^{i b}\right]+\frac{1}{\tilde{f}^{\sigma} \sqrt{h}} \partial_{a}\left[\tilde{f}^{\sigma} \sqrt{h} \mathcal{F}^{a b}\right]+\Lambda A^{b}=0 .
\end{aligned}
$$

To separate variables between $x$ - and $y$-spaces, we impose the ansatz

$$
A=\tilde{B}(y) C_{i}(x) d x^{i}+B(x) C_{a}(y) d y^{a} .
$$

There are three types of separable solutions (2.25):

(a) Vector fields on the $x$-space:

$$
A=\tilde{B}(y) C_{i}(x) d x^{i} .
$$

Substitution into the system (2.24) gives

$$
\begin{aligned}
\frac{1}{f^{\sigma} \sqrt{g}} \partial_{i}\left[f^{\sigma} \sqrt{g} \mathcal{F}^{i j}\right]+\left[\frac{1}{\tilde{f}^{\sigma} \sqrt{h}} \partial_{a}\left[\tilde{f}^{\sigma} \sqrt{h} h^{a b} \partial_{b} \tilde{B}\right]+\Lambda \tilde{B}\right] C^{j} & =0 \\
\frac{1}{f^{\sigma} \sqrt{g}} \partial_{i}\left[f^{\sigma} \sqrt{g} g^{i j} C_{j}\right] & =0 .
\end{aligned}
$$

\footnotetext{
${ }^{6}$ Note that since we are dealing with a massive vector field $A$, equations $(2.21)$ and $(2.22)$ are not invariant under the $A \rightarrow A+d f$ transformation.
} 
This leads to a system of two eigenvalue problems for decoupled ODEs:

$$
\frac{1}{\tilde{f}^{\sigma} \sqrt{h}} \partial_{a}\left[\tilde{f}^{\sigma} \sqrt{h} h^{a b} \partial_{b} \tilde{B}\right]+\tilde{\lambda}_{\text {scalar }} \tilde{B}=0, \quad \frac{1}{f^{\sigma} \sqrt{g}} \partial_{i}\left[f^{\sigma} \sqrt{g} \mathcal{C}^{i j}\right]+\lambda_{\text {vector }} C^{j}=0
$$

where $\mathcal{C}_{i j} \equiv \frac{1}{\widetilde{B}} \mathcal{F}_{i j}$ is the modified field strength corresponding to the potential $C_{i}$ :

$$
\mathcal{C}_{i j}=\partial_{i} C_{j}-\partial_{j} C_{i}+\zeta H_{i j k} C^{k}
$$

The system (2.27) leads to the eigenvalue

$$
\Lambda=\tilde{\lambda}_{\text {scalar }}+\lambda_{\text {vector }} .
$$

The vector field $C_{i}$ satisfies a constraint

$$
\frac{1}{f^{\sigma} \sqrt{g}} \partial_{i}\left[f^{\sigma} \sqrt{g} g^{i j} C_{j}\right]=0
$$

which ensures that the number of degrees of freedom covered by the ansatz (2.26) is $\left(\operatorname{dim}_{x}-1\right)$.

\section{(b) Vector fields on the $\boldsymbol{y}$-space:}

$$
A=B(x) \tilde{C}_{a}(y) d y^{a} .
$$

As before, substitution into the system (2.24) leads to a two eigenvalue problems for decoupled ODEs, ${ }^{7}$

$$
\frac{1}{f^{\sigma} \sqrt{g}} \partial_{i}\left[f^{\sigma} \sqrt{g} g^{i j} \partial_{j} B\right]+\lambda_{\text {scalar }} B=0, \quad \frac{1}{f^{\sigma} \sqrt{g}} \partial_{a}\left[f^{\sigma} \sqrt{h} \tilde{\mathcal{C}}^{a b}\right]+\lambda_{\text {vector }} \tilde{C}^{b}=0
$$

and the eigenvalue $\Lambda$ is given by

$$
\Lambda=\lambda_{\text {scalar }}+\tilde{\lambda}_{\text {vector }}
$$

The vector field $\tilde{C}_{a}$ satisfies a constraint

$$
\frac{1}{\tilde{f}^{\sigma} \sqrt{h}} \partial_{a}\left[\tilde{f}^{\sigma} \sqrt{h} h^{a b} \tilde{C}_{b}\right]=0
$$

so the ansatz $(2.26)$ describes $\left(\operatorname{dim}_{y}-1\right)$ degrees of freedom.

\section{(c) The scalar mode:}

$$
A=\tilde{B}(y) d C(x)+B(x) d \tilde{C}(y) .
$$

Substitution to the system (2.24) leads to the consistency conditions ${ }^{8}$

$$
C(x)=B(x), \quad \tilde{C}(y)=\mu \tilde{B}(y)
$$

\footnotetext{
${ }^{7}$ The field strength $\mathcal{C}_{a b}$ is defined by the counterpart of $(2.28), \tilde{\mathcal{C}}_{a b}=\partial_{a} \tilde{C}_{b}-\partial_{b} \tilde{C}_{a}+\zeta H_{a b c} \tilde{C}^{c}$, and it is related to the relevant components of $(2.22)$ by $\tilde{\mathcal{C}}_{a b}=\frac{1}{B} \mathcal{F}_{a b}$.

${ }^{8}$ We used the equations of motion for the three-form $H_{\mu \nu \lambda}$.
} 
with some constant $\mu$ and to system of ODEs:

$$
\begin{gathered}
\frac{1}{f^{\sigma} \sqrt{g}} \partial_{i}\left[f^{\sigma} \sqrt{g} g^{i j} \partial_{j} B\right]+\lambda_{\text {scalar }} B=0, \\
\frac{1}{\tilde{f}^{\sigma} \sqrt{h}} \partial_{a}\left[\tilde{f}^{\sigma} \sqrt{h} h^{a b} \partial_{b} \tilde{B}\right]+\tilde{\lambda}_{\text {scalar }} \tilde{B}=0 .
\end{gathered}
$$

The eigenvalue $\Lambda$ and the parameter $\mu$ are given by

$$
\Lambda=\lambda_{\text {scalar }}+\tilde{\lambda}_{\text {scalar }} \quad \mu=-\frac{\lambda_{\text {scalar }}}{\tilde{\lambda}_{\text {scalar }}} .
$$

If $\mu=1$, then the ansatz (2.35) describes a pure gauge: it gives $\Lambda=0$, but functions $(B, \tilde{B})$ remain arbitrary, and they are not constrained by the system $(2.37)$.

To summarize, the separable ansatz (2.25) describes

$$
\operatorname{dim}_{x}+\operatorname{dim}_{y}-1
$$

modes with non-zero values of $\Lambda$, and the eigenvalues are given by

$$
\Lambda=\left\{\tilde{\lambda}_{\text {scalar }}+\lambda_{\text {vector }}, \lambda_{\text {scalar }}+\tilde{\lambda}_{\text {scalar }}, \lambda_{\text {scalar }}+\tilde{\lambda}_{\text {vector }}\right\} .
$$

Therefore, to find the complete spectrum of the equation (2.21) on the product space (2.23), it is sufficient to determine the scalar and vector eigenvalues $\left(\lambda_{\text {scalar }}, \tilde{\lambda}_{\text {scalar }}, \lambda_{\text {vector }}, \tilde{\lambda}_{\text {vector }}\right)$ on the individual blocks. We will now solve this problem for $\mathrm{SO}(4)=\mathrm{SU}(2) \times \mathrm{SU}(2)$, and we will analyze $\mathrm{SO}(4) /[\mathrm{SO}(2) \times \mathrm{SO}(2)]=[\mathrm{SU}(2) / \mathrm{U}(1)]^{2}$ in section 2.5 .

\subsubsection{Vector modes on $\mathrm{SU}(2)$}

To evaluate $\left(\lambda_{\text {scalar }}, \tilde{\lambda}_{\text {scalar }}, \lambda_{\text {vector }}, \tilde{\lambda}_{\text {vector }}\right)$ and the corresponding eigenfunctions for $\mathrm{SO}(4)$, we recall the geometry $(2.12)$ in the $\left(y_{1}, y_{2}\right)$ coordinates. Comparing it to the general product space (2.23), we conclude the $f=\tilde{f}=1$, and that the scalar equations (2.37) reduce to (2.14) with identification

$$
\begin{aligned}
B & =e^{i n_{1}\left(\alpha_{L}-\beta_{L}\right)+i n_{2}\left(\alpha_{R}-\beta_{R}\right)} B_{1}\left(y_{1}\right), & \tilde{B} & =e^{i \tilde{n}_{1}\left(\alpha_{L}-\beta_{L}\right)+i \tilde{n}_{2}\left(\alpha_{R}-\beta_{R}\right)} B_{2}\left(y_{2}\right), \\
\lambda_{\text {scalar }} & =\frac{2 \pi}{k} \nu_{1}, & \tilde{\lambda}_{\text {scalar }} & =\frac{2 \pi}{k} \nu_{2} .
\end{aligned}
$$

Next we consider the vector equation from the system $(2.27),{ }^{9}$

$$
\frac{1}{\sqrt{g}} \partial_{i}\left[\sqrt{g} \mathcal{F}^{i j}\right]+\frac{2 \pi}{k} \lambda C^{j}=0, \quad \mathcal{F}_{i j}=\partial_{i} C_{j}-\partial_{j} C_{i}+\zeta H_{i j k} C^{k},
$$

on the relevant part of the geometry (2.12)

$$
\begin{aligned}
d s^{2} & =\frac{k}{2 \pi}\left[\frac{d y_{1}^{2}}{1-y_{1}^{2}}+\left(d \gamma_{L}\right)^{2}+\left(d \gamma_{R}\right)^{2}+2 y_{1} d \gamma_{L} d \gamma_{R}\right], \\
B & =\frac{k}{2 \pi}\left(1-y_{1}\right) d \gamma_{L} \wedge d \gamma_{R}, \quad \gamma_{L}=\alpha_{L}-\beta_{L}, \quad \gamma_{R}=\alpha_{R}-\beta_{R} .
\end{aligned}
$$

\footnotetext{
${ }^{9}$ To simplify the subsequent formulas, we rescaled the eigenvalue in (2.27) as $\lambda_{\text {vector }}=\frac{2 \pi}{k} \lambda$. Then $\lambda$ is analogous to $\left(\nu_{1}, \nu_{2}\right)$, and it will be equal to a product of integers or half-integers.
} 
The full analysis of the equation (2.42) is presented in the appendix A, and here we just outline the logic and write the final result.

(i) The most general separable solution for a vector field in the geometry (2.43) is given by

$$
C_{i} d x_{i}=e^{i n_{1} \gamma_{L}+i n_{2} \gamma_{R}}\left[V_{y} d y_{1}+V_{1} d \gamma_{L}+V_{2} d \gamma_{R}\right],
$$

where $\left(V_{y}, V_{1}, V_{2}\right)$ are functions of $y_{1}$, which are mixed in equations (2.42). We are looking for combinations of these components that satisfy decoupled equations, and to get insights into the structure of such combinations, we begin with studying the $\zeta=0$ case.

As demonstrated in the appendix A, the most general separable solutions of equations (2.42) with $\zeta=0$ are given by

$$
C_{i} d x_{i}=e^{i n_{1} \gamma_{L}+i n_{2} \gamma_{R}}\left[V_{y} d y_{1}+q_{+}\left(\hat{V}_{+}+\hat{V}_{-}\right) d \gamma_{L}+q_{-}\left(\hat{V}_{+}-\hat{V}_{-}\right) d \gamma_{R}\right],
$$

where

$$
q_{ \pm}=1 \pm \frac{n_{1}^{2}-n_{2}^{2}}{\left[\sqrt{\lambda-n_{1}^{2}}+\sqrt{\lambda-n_{2}^{2}}\right]^{2}}, \quad \frac{q_{-}}{q_{+}}=\left[\frac{\lambda-n_{2}^{2}}{\lambda-n_{1}^{2}}\right]^{1 / 2}
$$

Equations for the functions $\left(\hat{V}_{+}, \hat{V}_{-}\right)$decouple, and they are formulated as a system of eigenvalue problems

$$
\begin{aligned}
& \frac{d}{d y_{1}}\left[\frac{\left(1-y_{1}^{2}\right) \hat{V}_{+}^{\prime}}{\lambda y_{1}-n_{1} n_{2}+\mu}\right]+\frac{\lambda y_{1}-n_{1} n_{2}-\mu}{\lambda\left(y_{1}^{2}-1\right)} \hat{V}_{+}=0 \\
& \frac{d}{d y_{1}}\left[\frac{\left(1-y_{1}^{2}\right) \hat{V}_{-}^{\prime}}{\lambda y_{1}-n_{1} n_{2}-\mu}\right]+\frac{\lambda y_{1}-n_{1} n_{2}+\mu}{\lambda\left(y_{1}^{2}-1\right)} \hat{V}_{-}=0 .
\end{aligned}
$$

Here we defined $\mu$ as a convenient combination of constants $\left(\lambda, n_{1}, n_{2}\right)$ :

$$
\mu=\sqrt{\left(\lambda-n_{1}^{2}\right)\left(\lambda-n_{2}^{2}\right)}
$$

Note that, even though the modes $\hat{V}_{+}$and $\hat{V}_{-}$decouple, the function

$$
W_{+}=\frac{\left(1-y_{1}^{2}\right) \hat{V}_{+}^{\prime}}{\lambda y_{1}-n_{1} n_{2}+\mu}
$$

satisfies the same differential equation as $\hat{V}_{-}$. Similarly, a function $W_{-}$constructed from a derivative of $\hat{V}_{-}$satisfies the same equation as $\hat{V}_{+}$.

(ii) Interestingly, functions $\left(\hat{V}_{+}, \hat{V}_{-}\right)$can be expressed in terms of the solutions of the scalar equation (2.14). As demonstrated in the appendix A, any solution of equations (2.47) can be written as

$$
\hat{V}_{ \pm}\left(y_{1}\right)=C_{ \pm}\left[\left(1-y_{1}^{2}\right) B_{1}^{\prime}+\frac{1}{M}\left[\lambda y_{1}-n_{1} n_{2} \pm \mu\right] B_{1}\right]
$$


where function $B_{1}$ satisfies the differential equation (2.14), and parameters $\left(\lambda, \nu_{1}, M\right)$ are related by

$$
\lambda=M^{2}, \quad \nu_{1}=M(M+1), \quad M \geq 0 .
$$

In particular, this implies that the eigenvalues of the problem (A.12) are given by $\lambda=M^{2}$ with an integer $M$ which is subject to the constraint

$$
M \geq\left|n_{1}+n_{2}\right|+\left|n_{1}-n_{2}\right| .
$$

The last remaining component of the vector field, $V_{y}$ is given by

$$
\begin{aligned}
V_{y}= & i C_{+} \frac{n_{1} q_{-}+n_{2} q_{+}}{M}\left[B_{1}^{\prime}-\frac{n_{1} n_{2}-M^{2} y_{1}+\mu}{M\left(1-y_{1}^{2}\right)} B_{1}\right] \\
& +i C_{-} \frac{n_{2} q_{+}-n_{1} q_{-}}{M}\left[B_{1}^{\prime}-\frac{n_{1} n_{2}-M^{2} y_{1}-\mu}{M\left(1-y_{1}^{2}\right)} B_{1}\right] .
\end{aligned}
$$

(iii) To extend the solution $(2.45),(2.50),(2.51),(2.53)$ to arbitrary values of $\zeta$, we observe that two linear combinations of (2.50) are especially simple: the ones with

$$
C_{-}=C_{+} \quad \text { and } \quad C_{-}=-C_{+} .
$$

Let us begin with analyzing the first combination by setting $C_{-}=C_{+}=\frac{1}{2}$ :

$$
\begin{aligned}
\hat{V}_{+}-\hat{V}_{-} & =\frac{\mu}{M} B_{1}, \quad \hat{V}_{+}+\hat{V}_{-}=\left[\left(1-y_{1}^{2}\right) B_{1}^{\prime}+\frac{1}{M}\left[\lambda y_{1}-n_{1} n_{2}\right] B_{1}\right], \\
V_{y} & =\frac{i n_{2} q_{+}}{M}\left[B_{1}^{\prime}+\frac{\lambda y_{1}-n_{1} n_{2}}{M\left(1-y_{1}^{2}\right)} B_{1}\right]-\frac{i n_{1} q_{-}}{M^{2}} \frac{\mu}{\left(1-y_{1}^{2}\right)} B_{1} .
\end{aligned}
$$

Comparing with (2.45), we observe the $C_{\gamma_{R}}$ component is given by the scalar wavefunction $B_{1}$. This suggests that it might be useful to write the vector field $C_{i}$ in terms of the frames ${ }^{10}$

$$
e_{\mathbf{3}}^{\mu} \partial_{\mu}=\partial_{\gamma_{R}}, \quad e_{ \pm}^{\mu} \partial_{\mu}=-\frac{e^{\mp i \gamma_{R}}}{2 \sqrt{1-y_{1}^{2}}}\left[\left(1-y_{1}^{2}\right) \partial_{y_{1}} \pm i\left(y_{1} \partial_{\gamma_{R}}-\partial_{\gamma_{L}}\right)\right]
$$

Evaluating various projections of the field (2.55), we find remarkably simple relations:

$$
e_{\mathbf{3}}^{\mu} C_{\mu}=a_{3} e_{\mathbf{3}}^{\mu} \partial_{\mu} Z, \quad e_{+}^{\mu} C_{\mu}=a_{+} e_{+}^{\mu} \partial_{\mu} Z, \quad e_{-}^{\mu} C_{\mu}=a_{-} e_{-}^{\mu} \partial_{\mu} Z
$$

where $^{11}$

$$
Z=B_{1} e^{i n_{1} \gamma_{L}+i n_{2} \gamma_{R}}, \quad a_{3}=\frac{q_{+}\left(\lambda-n_{2}^{2}\right)}{i n_{2} M}, \quad a_{ \pm}=\frac{i\left(n_{2} \mp M\right) q_{+}}{M} .
$$

Note that the expressions (2.57) are reminiscent of the ansatz for solving the Maxwell's equations in the Myers-Perry geometry [31].

\footnotetext{
${ }^{10}$ Once $e_{3}^{\mu}$ is fixed by the observation above, the components of $e_{ \pm}^{\mu}$ are uniquely determined up to the overall factors.

${ }^{11}$ Recall that $q_{-} \mu=\left(\lambda-n_{2}^{2}\right) q_{+}$.
} 
(iv) The analysis of the second polarization, $C_{+}=-C_{-}=\frac{1}{2}$, can be performed in a similar fashion. In this case the convenient frames are

$$
\tilde{e}_{\mathbf{3}}^{\mu} \partial_{\mu}=\partial_{\gamma_{L}}, \quad \tilde{e}_{ \pm}^{\mu} \partial_{\mu}=-\frac{e^{ \pm i \gamma_{L}}}{2 \sqrt{1-y_{1}^{2}}}\left[\left(1-y_{1}^{2}\right) \partial_{y_{1}} \mp i\left(y_{1} \partial_{\gamma_{L}}-\partial_{\gamma_{R}}\right)\right]
$$

and the counterpart of the ansatz (2.57) with $\left(\tilde{e}_{\mathbf{3}}^{\mu}, \tilde{e}_{+}^{\mu}, \tilde{e}_{-}^{\mu}, \tilde{a}_{3}, \tilde{a}_{+}, \tilde{a}_{-}\right)$gives

$$
\tilde{a}_{3}=\frac{q_{-}\left(\lambda-n_{1}^{2}\right)}{i n_{1} M}, \quad \tilde{a}_{ \pm}=\frac{i\left(n_{1} \mp M\right) q_{-}}{M}, \quad \lambda=M^{2} .
$$

It turns out that to extend the results to non-zero values of $\zeta$, it is convenient to choose a different route. Writing the counterpart of the first line in (2.55) for $C_{+}=-C_{-}=\frac{1}{2}$,

$$
\hat{V}_{+}-\hat{V}_{-}=\left[\left(1-y_{1}^{2}\right) B_{1}^{\prime}+\frac{1}{M}\left[\lambda y_{1}-n_{1} n_{2}\right] B_{1}\right], \quad \hat{V}_{+}+\hat{V}_{-}=\frac{\mu}{M} B_{1}
$$

we observe that the function

$$
\tilde{B}_{1} \equiv-\frac{M}{\mu}\left[\left(1-y_{1}^{2}\right) B_{1}^{\prime}+\frac{1}{M}\left[\lambda y_{1}-n_{1} n_{2}\right] B_{1}\right]
$$

satisfies the scalar equation $(2.14)$ with $\tilde{\nu}_{1}=M(M-1)$. Furthermore, in terms of $\tilde{B}_{1}$, relations (2.61) become

$$
\hat{V}_{+}-\hat{V}_{-}=-\frac{\mu}{M} \tilde{B}_{1}, \quad \hat{V}_{+}+\hat{V}_{-}=\left[\left(1-y_{1}^{2}\right) \tilde{B}_{1}^{\prime}-\frac{1}{M}\left[\lambda y_{1}-n_{1} n_{2}\right] \tilde{B}_{1}\right] .
$$

These expressions can be obtained from (2.61) by a formal replacement

$$
B_{1} \rightarrow \tilde{B}_{1}, \quad M \rightarrow-M, \quad \nu_{1} \rightarrow \tilde{\nu}_{1}
$$

and the same replacement works for $V_{y}$ as well. Therefore, the $C_{-}=-C_{+}$polarization can still be described by the ansatz (2.57), but relations (2.58) should be replaced by

$$
Z=\tilde{B}_{1} e^{i n_{1} \gamma_{L}+i n_{2} \gamma_{R}}, \quad a_{3}=\frac{q_{+}\left(\lambda-n_{2}^{2}\right)}{i n_{2} M}, \quad a_{ \pm}=\frac{i\left(n_{2} \pm M\right) q_{+}}{M}, \quad M \geq 0 .
$$

Alternatively, we can keep only expressions (2.57)-(2.58), but allow parameter $M$ to take both positive and negative values. ${ }^{12}$

(v) In the case of general $\zeta$, we impose the ansatz (2.57) with

$$
Z=B_{1} e^{i n_{1} \gamma_{L}+i n_{2} \gamma_{R}}, \quad \nu_{1}=M(M+1),
$$

and undetermined constants $\left(a_{3}, a_{+}, a_{-}\right)$. In accordance with the discussion from item (iv), parameter $M$ can take positive and negative values, so to recover both

\footnotetext{
${ }^{12}$ Recall that $M$ was defined in (2.51) as a square root of $\lambda$.
} 
polarizations, every scalar mode is used twice. As demonstrated in the appendix A, the constants $\left(a_{3}, a_{+}, a_{-}\right)$obey the same relations (2.58),

$$
a_{ \pm}=\frac{n_{2}}{n_{2} \pm M} a_{3}
$$

even for a non-vanishing $\zeta$, but the expression for the eigenvalue $\lambda$ in terms of the parameter $M$ is modified as

$$
\lambda=M(M-\zeta) .
$$

In particular, for $\zeta= \pm 1$ the sets of scalar and vector eigenvalues are identical.

(vi) Finally, there are solutions with $\lambda_{\text {vector }}=0$, which correspond to a pure gauge:

$$
C_{i} d x^{i}=d f
$$

For $\zeta=0$, equations (2.42) are trivially satisfied since $F_{i j}=0$. In the case of a nonzero $\zeta$, the modified field strength does not vanish, but since $H_{i j k}$ is proportional to the volume form, the field $\mathcal{F}_{i j}$ is divergence-free:

$$
\mathcal{F}_{i j}=\zeta H_{i j k} C^{k} \Rightarrow \frac{1}{\sqrt{g}} \partial_{i}\left[\sqrt{g} \mathcal{F}^{i j}\right]=\frac{\zeta}{\sqrt{g}} \partial_{i}\left[\sqrt{g} H^{i j k} C_{k}\right]=\zeta H^{i j k} \partial_{i} C_{k}=0 .
$$

Therefore, equations (2.42) with $\lambda=0$ are satisfied by the vector field (2.69) with an arbitrary $f$. The ansatz (2.57) with $a_{3}=a_{+}=a_{-}$and an arbitrary function $Z$ covers all such solutions.

The construction described here gives the most general separable solution of equations (2.42), and a priori it is not obvious that the Lorentz constraint (2.26) would be satisfied. Remarkably, this constraint follows from the ansatz (2.57) and equations (2.42), without additional assumptions. This implies that the solution (2.66), (2.67), (2.68) can be used to build the vector modes on the product space $\mathrm{SO}(4)=\mathrm{SU}(2) \times \mathrm{SU}(2)$ using the procedure described in section 2.2.1.

\subsubsection{Summary of the vector fields on $\mathrm{SO}(4)$}

Let us now combine the discussion from sections 2.2.1 and 2.2.2 to describe separation of variables for vector fields on $\mathrm{SO}(4)$. We are looking for solutions of the eigenvalue problem (2.21) with $\mathcal{F}$ given by (2.22) on the geometry (2.12). In this case the $x$ and $y$ coordinates defined in (2.23) are given by

$$
x=\left\{y_{1}, \alpha_{L}-\beta_{L}, \alpha_{R}-\beta_{R}\right\}, \quad y=\left\{y_{2}, \alpha_{L}+\beta_{L}, \alpha_{R}+\beta_{R}\right\} .
$$

According to the general discussion from subsection 2.2.1, there are three types of separable vector modes:

(a) Vector fields on the $x$-space:

The ansatz for the vector field has the form

$$
A=\tilde{B}(y) C_{i}(x) d x^{i},
$$


Then the eigenvalue problem (2.21) ensures that the functions $\left(\tilde{B}, C_{i}\right)$ satisfy the system of differential equations (2.27) with the constraint (2.30).

In the case of $\mathrm{SO}(4)=\mathrm{SU}(2) \times \mathrm{SU}(2)$, the scalar function has the form

$$
\tilde{B}(y)=e^{i \tilde{n}_{1}\left(\alpha_{L}+\beta_{L}\right)+i \tilde{n}_{2}\left(\alpha_{R}+\beta_{R}\right)} B_{2}\left(y_{2}\right),
$$

and $B_{2}\left(y_{2}\right)$ satisfies the second equation in (2.14). The components of the vector field $C_{i}$ have the form (2.57),

$$
e_{\mathbf{3}}^{\mu} C_{\mu}=a_{3} e_{\mathbf{3}}^{\mu} \partial_{\mu} Z, \quad e_{+}^{\mu} C_{\mu}=a_{+} e_{+}^{\mu} \partial_{\mu} Z, \quad e_{-}^{\mu} C_{\mu}=a_{-} e_{-}^{\mu} \partial_{\mu} Z,
$$

with frames (2.56). Function $Z$ given by $(2.66),{ }^{13}$

$$
Z=B_{1}\left(y_{1}\right) e^{i n_{1} \gamma_{L}+i n_{2} \gamma_{R}},
$$

where $B_{1}$ is a solution of the first equation in (2.14) with $\nu_{1}=j_{1}\left(j_{1}+1\right)$. The eigenvalues of the vector equation (2.21) have the form (2.29),

$$
\Lambda=\tilde{\lambda}_{\text {scalar }}+\lambda_{\text {vector }}
$$

with

$$
\tilde{\lambda}_{\text {scalar }}=\frac{2 \pi}{k} \nu_{2}=\frac{2 \pi}{k} j_{2}\left(j_{2}+1\right), \quad \lambda_{\text {vector }}=\frac{2 \pi}{k} j_{1}\left(j_{1}-\zeta\right) .
$$

Note that for $\zeta= \pm 1$ the scalar and vector spectra, (2.15) and (2.76), are identical. The ansatz (2.72) describes two physical degrees of freedom.

\section{(b) Vector fields on the $\boldsymbol{y}$-space:}

This situation is analogous to the case (a) with a replacement

$$
x \rightarrow y, \quad \tilde{\lambda}_{\text {scalar }} \rightarrow \lambda_{\text {scalar }}, \quad \lambda_{\text {vector }} \rightarrow \tilde{\lambda}_{\text {vector }} .
$$

For example, the ansatz for the vector field is

$$
A=B(x) \tilde{C}_{a}(y) d y^{a} .
$$

and functions $\left(B, \tilde{C}_{a}\right)$ satisfy the system of differential equations (2.32) with the constraint (2.34). The vector $\tilde{C}_{a}$ and the scalar $B(x)$ are given by

$$
\begin{array}{ll}
\tilde{e}_{3}^{a} \tilde{C}_{a}=a_{3} e_{3}^{a} \partial_{a} \tilde{Z}, \quad \tilde{e}_{+}^{a} C_{a}=a_{+} \tilde{e}_{+}^{a} \partial_{a} \tilde{Z}, & \tilde{e}_{-}^{a} C_{a}=a_{-} \tilde{e}_{-}^{a} \partial_{a} \tilde{Z}, \\
B(x)=e^{i n_{1}\left(\alpha_{L}-\beta_{L}\right)+i n_{2}\left(\alpha_{R}-\beta_{R}\right)} B_{1}\left(y_{1}\right), & \tilde{Z}(y)=e^{i \tilde{n}_{1}\left(\alpha_{L}+\beta_{L}\right)+i \tilde{n}_{2}\left(\alpha_{R}+\beta_{R}\right)} B_{2}\left(y_{2}\right),
\end{array}
$$

and $\left(B_{1}\left(y_{1}\right), B_{2}\left(y_{2}\right)\right)$ satisfy equations (2.14). The eigenvalues of the equation (2.21) are

$$
\Lambda=\lambda_{\text {scalar }}+\tilde{\lambda}_{\text {vector }}
$$

with

$$
\lambda_{\text {scalar }}=\frac{2 \pi}{k} j_{1}\left(j_{1}+1\right), \quad \tilde{\lambda}_{\text {vector }}=\frac{2 \pi}{k} j_{2}\left(j_{2}-\zeta\right) .
$$

The ansatz (2.79) describes two physical degrees of freedom.

\footnotetext{
${ }^{13}$ Recall that $\gamma_{L}=\alpha_{L}-\beta_{L}, \gamma_{R}=\alpha_{R}-\beta_{R}$.
} 


\section{(c) The scalar mode:}

The ansatz for the gauge field is given by (2.35)-(2.36):

$$
A=\tilde{B}(y) d B(x)+\mu B(x) d \tilde{B}(y),
$$

and in the $\mathrm{SO}(4)$ case,

$$
B=e^{i n_{1}\left(\alpha_{L}-\beta_{L}\right)+i n_{2}\left(\alpha_{R}-\beta_{R}\right)} B_{1}\left(y_{1}\right), \quad \tilde{B}=e^{i \tilde{n}_{1}\left(\alpha_{L}+\beta_{L}\right)+i \tilde{n}_{2}\left(\alpha_{R}+\beta_{R}\right)} B_{2}\left(y_{2}\right) .
$$

Functions $B_{1}$ and $B_{2}$ satisfy equations (2.14), the eigenvalue $\Lambda$ and parameter $\mu$ are given by $(2.38)$

$$
\Lambda=\lambda_{\text {scalar }}+\tilde{\lambda}_{\text {scalar }}, \quad \mu=-\frac{\lambda_{\text {scalar }}}{\tilde{\lambda}_{\text {scalar }}} .
$$

As in the general case discussed in section 2.2.1, $\mu=1$ corresponds to a pure gauge, which gives $\Lambda=0$ and arbitrary functions $(B, \tilde{B})$ (the equations (2.14) are not required).

To summarize, application of the separable ansatz (2.25) to $\mathrm{SO}(4)$ describes five physical degrees of freedom, and the set of eigenvalues is given by

$$
\Lambda=\left\{\tilde{\lambda}_{\text {scalar }}+\lambda_{\text {vector }}, \lambda_{\text {scalar }}+\tilde{\lambda}_{\text {scalar }}, \lambda_{\text {scalar }}+\tilde{\lambda}_{\text {vector }}\right\} .
$$

The individual ingredients are specified by two numbers $\left(j_{1}, j_{2}\right)$, which can be either both integers or both half-integers:

$$
\begin{array}{ll}
\lambda_{\text {scalar }}=\frac{2 \pi}{k} j_{1}\left(j_{1}+1\right), & \tilde{\lambda}_{\text {scalar }}=\frac{2 \pi}{k} j_{2}\left(j_{2}+1\right) \\
\lambda_{\text {vector }}=\frac{2 \pi}{k} j_{1}\left(j_{1}-\zeta\right), & \tilde{\lambda}_{\text {vector }}=\frac{2 \pi}{k} j_{2}\left(j_{2}-\zeta\right) .
\end{array}
$$

The eigenvalues (2.86) have the standard degeneracy associated with $[\mathrm{U}(1)]^{4}$ quantum numbers $\left(n_{1}, n_{2}, \tilde{n}_{1}, \tilde{n}_{2}\right)$, but this degeneracy is enhances if $\zeta= \pm 1$ when all three ingredients of $(2.85)$ have the same $\left(j_{1}, j_{2}\right)$ dependence.

\subsection{Gauged WZW model for the $\mathrm{SO}(4) / \mathrm{SO}(2)$ coset}

Let us now gauge some of the symmetries of $\mathrm{SO}(4)$ and study various fields on the resulting backgrounds. In this subsection we go back to the group element (2.3) and gauge the $\mathrm{SO}(2)$ symmetry that acts as

$$
g \rightarrow h g h^{-1}, \quad \text { where } \quad h=\left[\begin{array}{cc}
q(\mu) & 0 \\
0 & I_{2 \times 2}
\end{array}\right] .
$$

Here $\mu$ is the gauge parameter. We choose a convenient gauge by setting

$$
X=q_{2}(\gamma) \operatorname{diag}\left(X_{1}, X_{2}\right), \quad \alpha_{R}=0, \quad \beta_{R}=0
$$


in the product (2.3). Then the metric, the dilaton, and the Kalb-Ramond field of the gauged WZW (gWZW) model become ${ }^{14}$

$$
\begin{aligned}
d s^{2}= & \frac{k}{\pi}\left[\frac{4 d X_{1}^{2}}{\left(1+X_{1}^{2}\right)^{2}}+\frac{4 d X_{2}^{2}}{\left(1+X_{2}^{2}\right)^{2}}+d \alpha^{2}+4 \frac{X_{1} X_{2} d \alpha d \beta}{X_{1}^{2}+X_{2}^{2}}+\frac{\left(1+X_{1}^{2} X_{2}^{2}\right) d \beta^{2}}{\left(X_{1}^{2}+X_{2}^{2}\right)}\right] \\
& +\frac{k}{\pi}\left[\frac{4\left(X_{1}^{2}-X_{2}^{2}\right)^{2} d \alpha d \gamma}{\left(1+X_{1}^{2}\right)\left(1+X_{2}^{2}\right)\left(X_{1}^{2}+X_{2}^{2}\right)}+\frac{4\left(X_{1}^{2}-X_{2}^{2}\right)^{2} d \gamma^{2}}{\left(1+X_{1}^{2}\right)\left(1+X_{2}^{2}\right)\left(X_{1}^{2}+X_{2}^{2}\right)}\right], \\
e^{-2 \phi}= & \frac{2\left(X_{1}^{2}+X_{2}^{2}\right)}{\left(1+X_{1}^{2}\right)\left(1+X_{2}^{2}\right)}, \quad \alpha=\alpha_{L}, \quad \beta=\beta_{L} .
\end{aligned}
$$

The geometry also contains a Kalb-Ramond $B$ field, but the expression for it in the $\left(X_{1}, X_{2}\right)$ coordinates is not very illuminating. In terms of coordinates $\left(y_{1}, y_{2}\right)$ introduced in (2.11), the geometry (2.90) becomes

$$
\begin{aligned}
d s^{2}= & \frac{k}{\pi}\left[d \alpha^{2}+\frac{2\left(y_{1}-y_{2}\right)}{y_{1}+y_{2}-2} d \alpha d \beta-\frac{4\left(y_{1}-1\right)\left(y_{2}-1\right)}{y_{1}+y_{2}-2} d \alpha d \gamma-\frac{4\left(y_{1}-1\right)\left(y_{2}-1\right)}{y_{1}+y_{2}-2} d \gamma^{2}\right] \\
& +\frac{k}{\pi}\left[-\frac{y_{1}+y_{2}+2}{y_{1}+y_{2}-2} d \beta^{2}+\frac{d y_{1}^{2}}{2-2 y_{1}^{2}}+\frac{d y_{2}^{2}}{2-2 y_{2}^{2}}\right], \\
H= & \frac{k}{\pi}\left\{(d \alpha+2 d \gamma) \wedge d \beta \wedge d\left[\frac{y_{2}-y_{1}}{y_{2}+y_{1}-2}\right]+d \alpha \wedge d \gamma \wedge d\left[\frac{2\left(y_{1} y_{2}-1\right)}{y_{2}+y_{1}-2}\right]\right\} \\
e^{-2 \phi}= & 2-y_{1}-y_{2} .
\end{aligned}
$$

To demonstrate separation of variables, we observe that the frames in the $(\alpha, \beta, \gamma)$ subspace can be chosen to be

$$
\begin{aligned}
e_{1}^{\mu} \partial_{\mu} & =\frac{1}{2} \sqrt{\frac{2-2 y_{1}}{1+y_{1}}}\left[\partial_{\alpha}-\partial_{\beta}+\frac{y_{1}}{1-y_{1}} \partial_{\gamma}\right], \\
e_{2}^{\mu} \partial_{\mu} & =\frac{1}{2} \sqrt{\frac{2-2 y_{2}}{1+y_{2}}}\left[\partial_{\alpha}+\partial_{\beta}+\frac{y_{2}}{1-y_{2}} \partial_{\gamma}\right], \\
e_{3}^{\mu} \partial_{\mu} & =\frac{2}{2}\left(\partial_{\alpha}-\partial_{\gamma}\right) .
\end{aligned}
$$

This implies that the inverse metric can be written as

$$
\frac{\pi}{k} g^{\mu \nu} \partial_{\mu} \partial_{\nu}=\left[2\left(1-y_{1}^{2}\right) \partial_{y_{1}}^{2}+e_{1}^{\mu} e_{1}^{\nu} \partial_{\mu} \partial_{\nu}\right]+\left[2\left(1-y_{2}^{2}\right) \partial_{y_{2}}^{2}+e_{2}^{\mu} e_{2}^{\nu} \partial_{\mu} \partial_{\nu}\right]+e_{3}^{\mu} e_{3}^{\nu} \partial_{\mu} \partial_{\nu}
$$

The first block depend only on $y_{1}$, the second block depends only on $y_{2}$, while the third block contains only constant coefficients. Such structure of frames, has also been encountered in Myers-Perry-AdS black holes in odd dimensions, where it guaranteed separation of the Helmholtz and Hamilton-Jacobi equations [10-24], as well as equations for the vector field [31] and higher forms [36]. In the present case, the structure (2.93) guarantees the full separation of variables in the Hamilton-Jacobi equation

$$
g^{\mu \nu} \frac{\partial S}{\partial x^{\mu}} \frac{\partial S}{\partial x^{\nu}}=\lambda
$$

\footnotetext{
${ }^{14}$ The general procedure for constructing the gWZW geometries and its application to the specific case (2.89) are discussed in the appendix B.
} 
but not in the Helmholtz equation (2.96). The obstacle comes from a non-separable determinant of the metric:

$$
\sqrt{g}=\left(\frac{k}{\pi}\right)^{\frac{5}{2}} \frac{1}{2-y_{1}-y_{2}} .
$$

It turns out that a modified Helmholtz equation

$$
e^{2 \sigma \phi} \nabla^{2}\left(e^{-2 \sigma \phi} \Phi\right)=-\Lambda \Phi
$$

is still separable if and only if $\sigma=1$. This special value of $\sigma$ has been already encountered in $[2,3]$, where the general formula for scalar eigenvalues $\Lambda$ on all $G / H$ gauged WZW models was derived using algebraic methods, which were applicable only to $\sigma=1$.

Substituting a separable ansatz for the scalar field,

$$
\Phi=\exp \left[i n_{1} \alpha+i n_{2} \beta+i n_{3}(\alpha+2 \gamma)\right] g_{1}\left(y_{1}\right) g_{2}\left(y_{2}\right),
$$

into the Helmholtz equation (2.96) in the geometry (2.90), and setting $\sigma=1$, we arrive at a system of ODEs:

$$
\begin{aligned}
& \frac{d}{d y_{1}}\left[\left(y_{1}^{2}-1\right) \frac{d g_{1}}{d y_{1}}\right]+\frac{1}{2}\left[\frac{n_{3}^{2}}{1-y_{1}}+\frac{\left(n_{1}-n_{2}\right)^{2}}{y_{1}+1}\right] g_{1}+\frac{1-\lambda_{1}^{2}}{4} g_{1}=0, \\
& \frac{d}{d y_{2}}\left[\left(y_{2}^{2}-1\right) \frac{d g_{2}}{d y_{2}}\right]+\frac{1}{2}\left[\frac{n_{3}^{2}}{1-y_{2}}+\frac{\left(n_{1}+n_{2}\right)^{2}}{y_{2}+1}\right] g_{2}+\frac{1-\lambda_{2}^{2}}{4} g_{2}=0 .
\end{aligned}
$$

The eigenvalue $\Lambda$ is given by

$$
\Lambda=\frac{2 \pi}{k}\left(\frac{\lambda_{1}^{2}}{4}+\frac{\lambda_{2}^{2}}{4}-\frac{1}{2}-\frac{n_{2}^{2}}{2}\right) .
$$

Equations (2.98) can be solved in terms of the hypergeometric function, and the result reads

$$
\begin{aligned}
& g_{1}=\left(1-y_{1}\right)^{\frac{n_{3}}{2}}\left(1+y_{1}\right)^{\frac{n_{-}}{2}} F\left[-k_{1}, 1+n_{3}+n_{-}+k_{1} ; 1+n_{3} ; \frac{1-y_{1}}{2}\right], \\
& g_{2}=\left(1-y_{2}\right)^{\frac{n_{3}}{2}}\left(1+y_{2}\right)^{\frac{n_{+}}{2}} F\left[-k_{2}, 1+n_{3}+n_{+}+k_{2} ; 1+n_{3} ; \frac{1-y_{2}}{2}\right], \\
& \Lambda=\frac{2 \pi}{k}\left[\frac{\left(2 k_{1}+n_{3}+n_{-}+1\right)^{2}}{4}+\frac{\left(2 k_{2}+n_{3}+n_{+}+1\right)^{2}}{4}-\frac{1+n_{2}^{2}}{2}\right] .
\end{aligned}
$$

Here we assumed that $n_{3} \geq 0$ and introduced two more non-negative parameters:

$$
n_{+}=\left|n_{1}+n_{2}\right|, \quad n_{-}=\left|n_{1}-n_{2}\right| .
$$

It is instructive to compare the expression for the eigenvalue from (2.100) with the general formula for the gauged WZW models on the $G / H$ cosets $[2,3]$. As demonstrated in [2, 3], the eigenfunctions of the scalar field (2.96) on such cosets are specified by a representations 
of the group $G$ and the subgroup $H$, and the eigenvalues are expressed in terms of the quadratic Casimirs of such representations: ${ }^{15}$

$$
\Lambda=\frac{2 \pi}{k} C_{2}\left(R_{G}\right)-\frac{2 \pi}{k} C_{2}\left(R_{H}\right) .
$$

To recover this formula, we rewrite the expression for $\Lambda$ from (2.100) in a suggestive form:

$$
\begin{aligned}
& \Lambda=\frac{2 \pi}{k}\left[j_{1}\left(j_{1}+1\right)+j_{2}\left(j_{2}+1\right)\right]-\frac{2 \pi}{k} \frac{n_{2}^{2}}{2}, \\
& j_{1}=k_{1}+\frac{n_{3}+n_{-}}{2}, \quad j_{2}=k_{2}+\frac{n_{3}+n_{+}}{2} .
\end{aligned}
$$

Note that in (2.18) and (2.19) we have already encountered the counterparts of the parameters $\left(j_{1}, j_{2}\right)$ in the $\mathrm{SO}(4)$ case. As expected the general formula (2.101) is reproduced.

\subsection{Vector fields on the $\mathrm{SO}(4) / \mathrm{SO}(2)$ gauged $\mathrm{WZW}$ model}

To separate variables in the vector equation (2.21)

$$
e^{2 \phi} \nabla_{\mu}\left[e^{-2 \phi} \mathcal{F}^{\mu \nu}\right]+4 \Lambda A^{\nu}=0, \quad \mathcal{F}_{\mu \nu}=\partial_{\mu} A_{\nu}-\partial_{\nu} A_{\mu}+\zeta H_{\mu \nu \sigma} A^{\sigma},
$$

we implement the idea that has been used to solve Maxwell's equations in the Myers-Perry geometry [31]. We begin with introducing complex combinations of frames (2.92) that depend only on $y_{1}$ or $y_{2}$, as well as the constant frame $e_{0}$ :

$$
e_{1 \pm}=e_{1} \pm i \tilde{e}_{1}, \quad e_{2 \pm}=e_{2} \pm i \tilde{e}_{2}, \quad e_{0}, \quad \tilde{e}_{1,2}=\sqrt{2\left(1-y_{1,2}^{2}\right)} \partial_{y_{1,2}} .
$$

Then we impose an ansatz inspired by our discussion in section 2.2 and by separation of vector equations in background of the Myers-Perry black holes [31]

$$
\begin{aligned}
e_{1 \pm}^{\mu} A_{\mu} & =b_{1 \pm}\left(y_{1}\right) e_{1 \pm}^{\mu} \partial_{\mu} \tilde{Z}, \quad e_{2 \pm}^{\mu} A_{\mu}=b_{2 \pm}\left(y_{2}\right) e_{2 \pm}^{\mu} \partial_{\mu} \tilde{Z}, \quad e_{0}^{\mu} A_{\mu}=b_{0} e_{0}^{\mu} \partial_{\mu} \tilde{Z} \\
\tilde{Z} & =e^{i n_{1} \alpha+i n_{2} \beta+i n_{3} \gamma} Z\left(y_{1}, y_{2}\right) .
\end{aligned}
$$

Direct substitution into (2.103) shows that the vector equations become separable only for $\zeta=1$, and the results are:

1. Coefficients $b_{1 \pm}$ and $b_{2 \pm}$ must be constant. This is consistent with constant eigenvalues of the modified Killing-Yano tensor corresponding to the metric (2.92),

$$
Y=\left(e^{2} \wedge \tilde{e}^{2}-e^{1} \wedge \tilde{e}^{1}\right) \wedge e^{0},
$$

which satisfies equations with twisted connections [25-28]:

$$
\begin{aligned}
\nabla_{n}^{+} Y_{m p q}+\nabla_{m}^{+} Y_{n p q} & =0, \quad \Gamma_{n p}^{d+}=\Gamma_{n p}^{d}+\frac{1}{2} H_{n p}^{d} \\
\nabla_{n}^{+} Y_{m p q} & =\partial_{n} Y_{m p q}-\Gamma_{n m}^{d+} Y_{d p q}-\Gamma_{n p}^{d+} Y_{m d q}-\Gamma_{n q}^{d+} Y_{m p d}
\end{aligned}
$$

\footnotetext{
${ }^{15}$ The expression in $[2,3]$ is slightly more general, but it reduces to $(2.101)$ in the geometric limit which we are discussing here. Also, the algebraic construction of [2,3] applies only to the equation (2.96) with $\sigma=1$, and it is remarkable that this equation separates and results in (2.100) precisely for this value of $\sigma$.
} 
Recall that in the case of the Myers-Perry geometry the metric and the KillingYano tensors had the form

$$
\begin{aligned}
d s^{2} & =\left[-l_{\mu}^{+} l_{\nu}^{-}+\sum_{i} m_{\mu}^{(i)+} m_{\nu}^{(i)-}+n_{\mu} n_{\nu}\right] d x^{\mu} d x^{\nu} \\
Y^{(2 n-k)} & =\star\left[\wedge h^{k}\right], \quad h=r l_{+} \wedge l_{-}+\sum_{i} x_{i} m_{+}^{(i)} \wedge m_{-}^{(i)}
\end{aligned}
$$

and the counterpart of the ansatz (2.105) was $^{16}$

$$
l_{ \pm}^{\mu} A_{\mu}= \pm \frac{1}{r \pm i \mu} \hat{l}_{ \pm} \Psi, \quad\left[m_{ \pm}^{(j)}\right]^{\mu} A_{\mu}=\mp \frac{i}{x_{j} \pm \mu} \hat{m}_{ \pm}^{(j)} \Psi \quad n^{\mu} A_{\mu}=\lambda \Psi .
$$

In particular, the prefactors in the last equation involved some combinations of the eigenvalues of $h$, and since the eigenvalues of (2.106) do not depend on $\left(y_{1}, y_{2}\right)$, the constant values of the coefficients $b_{1 \pm}$ and $b_{2 \pm}$ in (2.105) are not surprising.

2. Separable function $Z$ obeys a system of ODEs

$$
\begin{aligned}
& \frac{1}{1-y_{1}^{2}} \frac{\partial}{\partial y_{1}}\left[\left(1-y_{1}^{2}\right) \frac{\partial Z}{\partial y_{1}}\right]+\frac{\lambda_{1} Z}{1-y_{1}^{2}}-\frac{n_{3}^{2} Z}{4\left(1-y_{1}^{2}\right)^{2}}-\frac{\left(n_{1}-n_{2}\right)\left(n_{1}-n_{2}-n_{3}\right) Z}{2\left(1-y_{1}\right)\left(1-y_{1}^{2}\right)^{2}}=0 \\
& \frac{1}{1-y_{2}^{2}} \frac{\partial}{\partial y_{2}}\left[\left(1-y_{2}^{2}\right) \frac{\partial Z}{\partial y_{2}}\right]+\frac{\lambda_{2} Z}{1-y_{2}^{2}}-\frac{n_{3}^{2} Z}{4\left(1-y_{2}^{2}\right)^{2}}-\frac{\left(n_{1}+n_{2}\right)\left(n_{1}+n_{2}-n_{3}\right) Z}{2\left(1-y_{2}\right)\left(1-y_{2}^{2}\right)^{2}}=0 .
\end{aligned}
$$

3. The eigenvalues $\Lambda$ of (2.103) are given by

$$
\Lambda=\frac{2 \pi}{k}\left(\lambda_{1}+\lambda_{2}-\frac{n_{2}^{2}}{2}\right) .
$$

4. Five coefficients $\left(b_{1 \pm}, b_{2 \pm}, b_{0}\right)$ obey one constraint:

$$
\begin{aligned}
& \left(b_{1+}+b_{1-}\right)\left[4 \lambda_{1}+1\right]+\left(b_{2+}+b_{2-}\right)\left[4 \lambda_{2}+1\right]+4 b_{0}\left(n_{1}-n_{3}\right)^{2}- \\
& \quad-\sum_{ \pm} b_{1 \pm}\left(n_{2}+n_{3}-n_{1} \pm 1\right)^{2}-\sum_{ \pm} b_{2 \pm}\left(n_{3}-n_{1}-n_{2} \pm 1\right)^{2}=0 .
\end{aligned}
$$

This constraint follows from the equations (2.103), and it also ensures the Lorenz condition

$$
\nabla_{\mu}\left[e^{-2 \phi} A^{\mu}\right]=0 .
$$

The metric (2.92) also admits another modified Killing-Yano tensor:

$$
\hat{Y}=\left(\hat{e}^{2} \wedge \tilde{e}^{2}-\hat{e}^{1} \wedge \tilde{e}^{1}\right) \wedge \hat{e}^{0},
$$

where new frames $\hat{e}^{2}, \hat{e}^{0}$ and $\hat{e}^{1}$ are

$$
\begin{aligned}
& \hat{e}^{1}=\frac{\sqrt{2-2 y_{1}^{2}}}{2-y_{1}-y_{2}}\left(d \beta+\left(1-y_{2}\right) d \gamma\right), \quad \hat{e}^{2}=-\frac{\sqrt{2-2 y_{2}^{2}}}{2-y_{1}-y_{2}}\left(d \beta+\left(1-y_{1}\right) d \gamma\right), \\
& \hat{e}^{0}=d \alpha-\frac{y_{1}-y_{2}}{2-y_{1}-y_{2}} d \beta+\frac{2\left(1-y_{1}\right)\left(1-y_{2}\right)}{2-y_{1}-y_{2}} d \gamma .
\end{aligned}
$$

\footnotetext{
${ }^{16}$ See [31] for details and derivation.
} 
In contrast to (2.106) this modified Killing-Yano tensor satisfies equations with different twisted connections:

$$
\begin{aligned}
\nabla_{n}^{-} \hat{Y}_{m p q}+\nabla_{m}^{-} \hat{Y}_{n p q} & =0, \quad \Gamma_{n p}^{d-}=\Gamma_{n p}^{d}-\frac{1}{2} H_{n p}^{d} \\
\nabla_{n}^{-} \hat{Y}_{m p q} & =\partial_{n} \hat{Y}_{m p q}-\Gamma_{n m}^{d-} \hat{Y}_{d p q}-\Gamma_{n p}^{d-} \hat{Y}_{m d q}-\Gamma_{n q}^{d-} \hat{Y}_{m p d}
\end{aligned}
$$

Therefore another separable ansatz of the vector field equation is possible by replacing $e^{1}$, $e^{2}$ and $e^{0}$ in (2.105) by $\hat{e}^{1}, \hat{e}^{2}$ and $\hat{e}^{0}$, respectively. A direct substitution of this alternative ansatz into (2.103) shows the vector equation becomes separable when $\zeta=-1$. In the appendix B we will show these two possible separable ansatz are related to the left and right frames of the gWZW model.

To summarize, in this subsection we have demonstrated separability of the twisted vector equation (2.103) for two values of the twisting parameter: $\zeta= \pm 1$. In both cases the components of the vector field are given by (2.105), but the frames used in these relations are different: $\zeta=1$ corresponds to the left-invariant forms, and $\zeta=-1$ corresponds to the right-invariant ones. The separation of the vector equation is not possible for any other values of $\zeta$, in particular, the standard equation corresponding to $\zeta=0$ does not separate. In the cases when separation is possible, the eigenvalues (2.109) are equal to their scalar counterparts (2.100), so the group theoretic formula (2.101) which has been derived for the scalar spectrum, seems to be applicable to vectors with $\zeta= \pm 1$ as well. We have already encountered this phenomenon in section 2.2.2, where the scalar and vector spectra (2.87) agreed precisely for $\zeta= \pm 1$.

\subsection{Scalars and vectors on the $\frac{\mathrm{SO}(4)}{\mathrm{SO}(2) \times \mathrm{SO}(2)}$ gauged $\mathrm{WZW}$ model}

Let us now gauge one more $\mathrm{U}(1)$ isometry and study various fields on the resulting $\mathrm{SO}(4) /[\mathrm{SO}(2) \times \mathrm{SO}(2)]$ coset. To do so, we go back to the group element (2.3) and gauge the $\mathrm{SO}(2) \times \mathrm{SO}(2)$ subgroup that acts as

$$
g \rightarrow h g h^{-1}, \quad \text { where } \quad h=\left[\begin{array}{cc}
q_{2}(\mu) & 0 \\
0 & q_{2}(\nu)
\end{array}\right]
$$

This leads to the shifts

$$
\alpha_{L, R} \rightarrow \alpha_{L, R} \pm \mu, \quad \beta_{L, R} \rightarrow \beta_{L, R} \pm \nu
$$

in the parameters of (2.3), and the gauge can be fixed by setting $\alpha_{R}=\beta_{R}=0$. The resulting coset element has the form

$$
g=\left[\begin{array}{cc}
q_{2}(\alpha) & 0 \\
0 & q_{2}(\beta)
\end{array}\right]\left[\begin{array}{cc}
I-\frac{2}{1+X X^{T}} X X^{T} & \frac{2}{1+X X^{T}} X \\
-X^{T} \frac{2}{1+X X^{T}} & I-\frac{2}{1+X^{T} X^{T}} X^{T} X
\end{array}\right], \quad X=\operatorname{diag}\left(X_{1}, X_{2}\right)
$$


Using the general procedure for constructing the metric of the gauged WZW model, ${ }^{17}$ we arrive at the geometry

$$
\begin{aligned}
d s^{2}=\frac{k}{\pi}[ & \left.\frac{4 d X_{1}^{2}}{\left(1+X_{1}^{2}\right)^{2}}+\frac{4 d X_{2}^{2}}{\left(1+X_{2}\right)^{2}}+\frac{4 X_{1} X_{2}\left(1+X_{1}^{2}\right)\left(1+X_{2}^{2}\right)}{\left(X_{1}^{2}-X_{2}^{2}\right)^{2}} d \alpha d \beta\right] \\
& +\frac{k}{\pi}\left[\frac{X_{2}^{2}+X_{1}^{2}\left(1+4 X_{2}^{2}+X_{1}^{2} X_{2}^{2}+X_{2}^{4}\right)}{\left(X_{1}^{2}-X_{2}^{2}\right)^{2}}\left(d \alpha^{2}+d \beta^{2}\right)\right], \\
e^{-2 \phi}= & \frac{4\left(X_{1}^{2}-X_{2}^{2}\right)^{2}}{\left(1+X_{1}^{2}\right)^{2}\left(1+X_{2}^{2}\right)^{2}} .
\end{aligned}
$$

In contrast to the $\mathrm{SO}(4) / \mathrm{SO}(2)$ coset, the geometry (2.118) does not contain a $B$ field. A sequence of invertible maps,

$$
X_{i}=\frac{i\left(1-w_{i}\right)}{1+w_{i}}, \quad w_{1}=\sqrt{x_{1} x_{2}}, \quad w_{2}=\sqrt{\frac{x_{2}}{x_{1}}}, \quad y_{i}=\frac{1+x_{i}^{2}}{2 x_{i}}
$$

leads to a separable form of the metric:

$$
\begin{aligned}
d s^{2} & =\frac{k}{2 \pi}\left(\frac{1+y_{1}}{1-y_{1}}[d \alpha-d \beta]^{2}+\frac{1+y_{2}}{1-y_{2}}[d \alpha+d \beta]^{2}+\frac{d y_{1}^{2}}{1-y_{1}^{2}}+\frac{d y_{2}^{2}}{1-y_{2}^{2}}\right) . \\
e^{-2 \phi} & =\left(1-y_{1}\right)\left(1-y_{2}\right), \quad \sqrt{g}=\left(\frac{k}{2 \pi}\right)^{2} \frac{1}{\left(1-y_{1}\right)\left(1-y_{2}\right)} .
\end{aligned}
$$

In contrast to the situations discussed in section 2.3, the scalar equation (2.96) separates for all values of $\sigma$. This is not surprising since the geometry (2.120) describes two copies of $\mathrm{SU}(2) / \mathrm{U}(1)$ :

$$
\frac{\mathrm{SO}(4)}{\mathrm{SO}(2) \times \mathrm{SO}(2)}=\frac{\mathrm{SU}(2)_{L} \times \mathrm{SU}(2)_{R}}{\mathrm{U}(1)_{L} \times \mathrm{U}(1)_{R}}=\frac{\mathrm{SU}(2)_{L}}{\mathrm{U}(1)_{L}} \times \frac{\mathrm{SU}(2)_{R}}{\mathrm{U}(1)_{R}} .
$$

Imposing a separable ansatz

$$
\Phi=e^{i n_{1}(\alpha-\beta)+i n_{2}(\alpha+\beta)} Y_{1}\left(y_{1}\right) Y_{2}\left(y_{2}\right),
$$

and substituting the result into (2.96), we arrive at a system of two ODEs:

$$
\begin{aligned}
& \frac{1}{\left(1-y_{1}\right)^{\sigma}} \frac{d}{d y_{1}}\left[\left(1-y_{1}\right)^{\sigma}\left(1+y_{1}\right) \frac{d Y_{1}}{d y_{1}}\right]-\frac{n_{1}^{2}}{1+y_{1}} Y_{1}+\frac{\lambda_{1}}{1-y_{1}} Y_{1}=0 \\
& \frac{1}{\left(1-y_{2}\right)^{\sigma}} \frac{d}{d y_{2}}\left[\left(1-y_{2}\right)^{\sigma}\left(1+y_{2}\right) \frac{d Y_{2}}{d y_{2}}\right]-\frac{n_{2}^{2}}{1+y_{2}} Y_{2}+\frac{\lambda_{2}}{1-y_{2}} Y_{2}=0 .
\end{aligned}
$$

The eigenvalues of the full problem (2.96) are $\Lambda=\frac{2 \pi}{k}\left(\lambda_{1}+\lambda_{2}\right)$.

Equations (2.123) can be solved in terms of the hypergeometric functions:

$$
\begin{aligned}
& Y_{1}=\left(1+y_{1}\right)^{-n_{1}} F\left[-k_{1}, k_{1}-2 n_{1}+\sigma ; \sigma ; \frac{1-y_{1}}{2}\right], \\
& Y_{2}=\left(1+y_{2}\right)^{-n_{2}} F\left[-k_{2}, k_{2}-2 n_{2}+\sigma ; \sigma ; \frac{1-y_{2}}{2}\right] .
\end{aligned}
$$

\footnotetext{
${ }^{17}$ See appendix B for the details.
} 
The eigenvalues $\Lambda$ in equation (2.96) are given by

$$
\Lambda=\frac{2 \pi}{k}\left(\lambda_{1}+\lambda_{2}\right), \quad \lambda_{1,2}=\left(k_{1,2}-n_{1,2}+\frac{\sigma}{2}\right)^{2}-n_{1,2}^{2}-\frac{\sigma^{2}}{4} .
$$

Regularity requires $k_{1}$ and $k_{2}$ to be non-negative integers. Setting $\sigma=1$ and introducing $j_{1,2}=k_{1,2}-n_{1,2}$ the eigenvalues can be written as

$$
\lambda_{1,2}=j_{1,2}\left(j_{1,2}+1\right)-n_{1,2}^{2} .
$$

This leads to the expressions for $\Lambda_{ \pm}$which are consistent with an application of the general formula (2.101) for a coset $[2,3]$ to the $\mathrm{SU}(2) / \mathrm{U}(1)$ case. Equation (2.125) also hints at a potential generalization of the formula (2.101) to arbitrary values of $\sigma$. Such generalization indeed exists for all groups and cosets, and it will be discussed elsewhere [6].

The eigenfunctions of the vector field (2.21), (2.22) follow the pattern outlined in section 2.2.1. In the present case there is no $H$-field, so one does not have to consider $\zeta$-modified vector equations, and the analysis becomes simpler than the one presented in sections 2.2.2, 2.2.3.

Division of space (2.120) into two blocks,

$$
x=\left\{y_{1}, \alpha-\beta\right\}, \quad y=\left\{y_{2}, \alpha+\beta\right\} .
$$

and application of the general pattern presented in section 2.2.1 leads to three types of vector modes:

(a) Vector fields on the $x$-space:

The ansatz for the vector field has the form

$$
A=\tilde{B}(y) C_{i}(x) d x^{i},
$$

and the eigenvalue problem (2.21) leads to the (2.27) for the functions $\left(\tilde{B}, C_{i}\right)$. Field $C_{i}$ must satisfy the constraint $(2.30)$ as well, but as we will see, in the $\frac{\mathrm{SO}(4)}{\mathrm{SO}(2) \times \mathrm{SO}(2)}$ case this does not lead to additional restrictions.

In the present case, the scalar function has the form

$$
\tilde{B}(y)=e^{i n_{2}(\alpha+\beta)} Y_{2}\left(y_{2}\right),
$$

and $Y_{2}\left(y_{2}\right)$ satisfies the second equation (2.123):

$$
\frac{1}{\left(1-y_{2}\right)^{\sigma}} \frac{d}{d y_{2}}\left[\left(1-y_{2}\right)^{\sigma}\left(1+y_{2}\right) \frac{d Y_{2}}{d y_{2}}\right]-\frac{n_{2}^{2}}{1+y_{2}} Y_{2}+\frac{\tilde{\lambda}_{\text {scalar }}}{1-y_{2}} Y_{2}=0 .
$$

The vector field $C_{i}$ has the form

$$
C_{i} d x^{i}=e^{i n_{1}(\alpha-\beta)}\left[V_{1} d y_{1}+V_{-}(d \alpha-d \beta)\right] .
$$

where $V_{1}$ and $V_{-}$are functions of $y_{1}$. Substitution into the second equation in (2.27) gives and expression for $V_{1}$,

$$
V_{1}=\frac{i n_{1}\left(1-y_{1}\right) V_{-}^{\prime}}{\lambda_{\text {vector }}\left(1+y_{1}\right)-n_{1}^{2}\left(1-y_{1}\right)},
$$


as well as a differential equation for $V_{-}$:

$$
\frac{1+y_{1}}{\left(1-y_{1}\right)^{\sigma}} \frac{d}{d y_{1}}\left[\frac{\left(1-y_{1}\right)^{\sigma+1}\left(1+y_{1}\right) V_{-}^{\prime}}{\lambda_{\text {vector }}\left(1+y_{1}\right)-n_{1}^{2}\left(1-y_{1}\right)}\right]+V_{-}=0 .
$$

The Lorenz condition (2.30),

$$
\partial_{i}\left[e^{-2 \sigma \phi} \sqrt{g} g^{i j} C_{j}\right]=0
$$

is automatically satisfied, and the eigenvalues of the problem (2.21) are given by

$$
\Lambda=\tilde{\lambda}_{\text {scalar }}+\lambda_{\text {vector }} \text {. }
$$

Interestingly, (2.132) and the first equation in (2.123) have the same set of eigenvalues, and solutions $V_{-}$can be written in terms of eigenfunctions $Y_{1}$ by

$$
V_{-}=\left(1+y_{1}\right) \frac{d Y_{1}}{d y_{1}}-\frac{\left(n_{1}\right)^{2} \sigma}{\lambda} Y_{1}, \quad \lambda_{\text {vector }}=\lambda_{\text {scalar }} .
$$

(b) Vector fields on the $\boldsymbol{y}$-space:

This situation is analogous to the case (a) with a replacement

$$
x \rightarrow y, \quad \tilde{\lambda}_{\text {scalar }} \rightarrow \lambda_{\text {scalar }}, \quad \lambda_{\text {vector }} \rightarrow \tilde{\lambda}_{\text {vector }} .
$$

The ansatz for the vector field is

$$
A=B(x) \tilde{C}_{a}(y) d y^{a} .
$$

with

$$
B(x)=e^{i n_{1}(\alpha-\beta)} Y_{1}\left(y_{1}\right), \quad \tilde{C}_{a} d x^{a}=e^{i n_{2}(\alpha+\beta)}\left[V_{2} d y_{2}+V_{+}(d \alpha+d \beta)\right] .
$$

Function $Y_{1}\left(y_{1}\right)$ satisfies the first ODE from (2.123),

$$
\frac{1}{\left(1-y_{1}\right)^{\sigma}} \frac{d}{d y_{1}}\left[\left(1-y_{1}\right)^{\sigma}\left(1+y_{1}\right) \frac{d Y_{1}}{d y_{1}}\right]-\frac{n_{1}^{2}}{1+y_{1}} Y_{1}+\frac{\lambda_{\text {scalar }}}{1-y_{1}} Y_{1}=0
$$

and $V_{+}$satisfies a counterpart of $(2.132)$

$$
\frac{1+y_{2}}{\left(1-y_{2}\right)^{\sigma}} \frac{d}{d y_{2}}\left[\frac{\left(1-y_{2}\right)^{\sigma+1}\left(1+y_{2}\right) V_{+}^{\prime}}{\tilde{\lambda}_{\text {vector }}\left(1+y_{2}\right)-n_{2}^{2}\left(1-y_{2}\right)}\right]+V_{+}=0 \text {. }
$$

As in the case (a), the sets of scalar and vector eigenvalues, $\left\{\tilde{\lambda}_{\text {scalar }}\right\}$ and $\left\{\tilde{\lambda}_{\text {vector }}\right\}$, are the same, and the eigenfunctions of (2.139) and (2.123) are related by

$$
V_{+}=\left(1+y_{2}\right) \frac{d Y_{2}}{d y_{2}}-\frac{\left(n_{2}\right)^{2} \sigma}{\tilde{\lambda}_{\text {vector }}} Y_{2}, \quad \lambda_{\text {vector }}=\lambda_{\text {scalar }} .
$$

Function $V_{2}$ is given by

$$
V_{2}=\frac{i n_{2}\left(1-y_{2}\right) V_{+}^{\prime}}{\tilde{\lambda}_{\text {vector }}\left(1+y_{2}\right)-n_{2}^{2}\left(1-y_{2}\right)},
$$

and the eigenvalues of the problem (2.21) are

$$
\Lambda=\frac{2 \pi}{k}\left(\lambda_{\text {scalar }}+\tilde{\lambda}_{\text {scalar }}\right) \text {. }
$$




\section{(c) The scalar mode:}

The ansatz for the gauge field is given by (2.35)-(2.36):

$$
A=\tilde{B}(y) d B(x)+\mu B(x) d \tilde{B}(y)
$$

and in the present case,

$$
B(x)=e^{i n_{1}(\alpha-\beta)} Y_{1}\left(y_{1}\right), \quad \tilde{B}(y)=e^{i n_{2}(\alpha+\beta)} Y_{2}\left(y_{2}\right) .
$$

Functions $Y_{1}$ and $Y_{2}$ satisfy equations (2.123), and the eigenvalue $\Lambda$ and parameter $\mu$ are given by (2.38)

$$
\Lambda=\frac{2 \pi}{k}\left(\lambda_{1}+\lambda_{2}\right), \quad \mu=-\frac{\lambda_{1}}{\lambda_{2}} .
$$

To summarize, application of the separable ansatz $(2.25)$ to $\frac{\mathrm{SO}(4)}{\mathrm{SO}(2) \times \mathrm{SO}(2)}$ describes three physical degrees of freedom per each pair of eigenvalues $\left(\lambda_{1}, \lambda_{2}\right)$ of the system $(2.123)$. The full spectrum describes three copies of (2.125) corresponding to cases (a), (b) and (c).

Our analysis was based on the product structure of the space (2.121), but it is also instructive to compare with the ansatz (2.105) inspired by Maxwell's equation on black hole geometries. To do so, we write the metric (2.120) in terms of frames:

$$
\begin{aligned}
d s^{2} & =\frac{k}{2 \pi}\left(e_{\mu}^{1+} e_{\nu}^{1-} d x^{\mu} d x^{\nu}+e_{\mu}^{2+} e_{\nu}^{2-} d x^{\mu} d x^{\nu}\right), \\
e_{\mu}^{1 \pm} d x^{\mu} & =\sqrt{\frac{1}{1-y_{1}^{2}}}\left[d y_{1} \pm i\left(1+y_{1}\right)(d \alpha+d \beta)\right], \\
e_{\mu}^{2 \pm} d x^{\mu} & =\sqrt{\frac{1}{1-y_{2}^{2}}}\left[d y_{2} \pm i\left(1+y_{2}\right)(d \alpha-d \beta)\right], \\
e_{1 \pm}^{\mu} \partial_{\mu} & =\sqrt{1-y_{1}^{2}}\left[\partial_{y_{1}} \mp \frac{i}{\left(1+y_{1}\right)} \partial_{\alpha+\beta}\right], \quad e_{2 \pm}^{\mu} \partial_{\mu}=\sqrt{1-y_{2}^{2}}\left[\partial_{y_{2}} \mp \frac{i}{\left(1+y_{2}\right)} \partial_{\alpha-\beta}\right] .
\end{aligned}
$$

Then equations (2.128), (2.130), (2.131), (2.134), lead to simple expressions for the projections:

$$
\begin{array}{rlrl}
\text { (a) : } e_{1+}^{\mu} A_{\mu} & =-\frac{i\left(\lambda_{1}-n_{1} \sigma\right)}{\lambda_{1}} e_{1+}^{\mu} \partial_{\mu} Z, \quad e_{1-}^{\mu} A_{\mu} & =\frac{i\left(\lambda_{1}+n_{1} \sigma\right)}{\lambda_{1}} e_{1-}^{\mu} \partial_{\mu} Z, \\
e_{2 \pm}^{\mu} A_{\mu} & =0, & Z & =e^{i n_{1}(\alpha-\beta)+i n_{2}(\alpha+\beta)} Y_{1}\left(y_{1}\right) Y_{2}\left(y_{2}\right) .
\end{array}
$$

Here we used the first equation form (2.123) to eliminate higher derivatives of $Y_{1}\left(y_{1}\right)$. Similarly, for the other branches we find:

$$
\begin{aligned}
\text { (b) : } \quad e_{2+}^{\mu} A_{\mu} & =-\frac{i\left(\lambda_{2}-n_{2} \sigma\right)}{\lambda_{2}} e_{2+}^{\mu} \partial_{\mu} Z, \quad e_{2-}^{\mu} A_{\mu}=\frac{i\left(\lambda_{2}+n_{2} \sigma\right)}{\lambda_{2}} e_{2-}^{\mu} \partial_{\mu} Z, \\
e_{1 \pm}^{\mu} A_{\mu} & =0, \\
\text { (c) }: & e_{1 \pm}^{\mu} A_{\mu}=e_{1 \pm}^{\mu} \partial_{\mu} Z, \quad e_{2 \pm}^{\mu} A_{\mu}=-\frac{\lambda_{1}(\alpha-\beta)+i n_{2}(\alpha+\beta)}{\lambda_{2}} e_{2 \pm}^{\mu} \partial_{1}\left(y_{1}\right) Y_{2}\left(y_{2}\right) ;
\end{aligned}
$$


All three cases, as well as their arbitrary linear combinations, match the structure (2.105)

$$
e_{1 \pm}^{\mu} A_{\mu}=b_{1 \pm} e_{1 \pm}^{\mu} \partial_{\mu} \tilde{Z}, \quad e_{2 \pm}^{\mu} A_{\mu}=b_{2 \pm} e_{2 \pm}^{\mu} \partial_{\mu} \tilde{Z}
$$

with constant coefficients $\left(b_{1 \pm}, b_{2 \pm}\right)$.

To summarize, in this subsection we demonstrated a full separation of variables in the scalar and vector equations on the background of the WZW model for the $\mathrm{SO}(4) /[\mathrm{SO}(2) \times$ $\mathrm{SO}(2)]$ coset. We found that, up to an extra degeneracy in the vector sector, the scalar and vector spectra are identical and the eigenvalues are given by

$$
\Lambda=\frac{2 \pi}{k}\left(\lambda_{1}+\lambda_{2}\right), \quad \lambda_{1,2}=j_{1,2}\left(j_{1,2}+1\right)-n_{1,2}^{2} .
$$

The components of the vector field are expressed in terms of the scalar by one of the options (2.147)-(2.148), and various ingredients of the scalar eigenfunction (2.122) satisfy ordinary differential equations (2.123).

\subsection{Gauging and T-duality}

In this section we have analyzed the eigenvalues problems for scalar and vector fields on the backgrounds of the (gauged) WZW models corresponding to $\mathrm{SO}(4)$ and its cosets, $\mathrm{SO}(4) / H$. Although the differential equations describing the dynamical excitations varied with the subgroup $H$, there were some similarities between them, and in this subsection we will address the origin of these similarities. Specifically, we will demonstrate that the target spaces of various $\mathrm{SO}(4) / H$ are related to each other by $\mathrm{T}$ duality, and that equations for excitations transform under such dualities in a simple way.

We begin with the $\mathrm{SO}(4)$ WZW model that produces the geometry (2.12). Defining new coordinates $\left(\alpha_{ \pm}, \beta_{ \pm}\right)$by

$$
\alpha_{ \pm}=\frac{\left(\alpha_{L}-\beta_{L}\right) \pm\left(\alpha_{R}-\beta_{R}\right)}{2}, \quad \beta_{ \pm}=\frac{\left(\alpha_{+}+\beta_{L}\right) \pm\left(\alpha_{L}-\beta_{L}\right)}{2},
$$

we can write the $B$ field and the angular parts of the metric as

$$
\begin{aligned}
d s^{2} & =\left(1+y_{1}\right) d \alpha_{+}^{2}+\left(1-y_{1}\right) d \alpha_{-}^{2}+\left(1+y_{2}\right) d \beta_{+}^{2}+\left(1-y_{2}\right) d \beta_{-}^{2}, \\
B & =\left(1-y_{2}\right) d \beta_{+} \wedge d \beta_{-}+\left(1-y_{1}\right) d \alpha_{+} \wedge d \alpha_{-} .
\end{aligned}
$$

To simplify the discussion, we rescaled the metric and the $B$-field by the factor $\frac{k}{2 \pi}$. Performing T-dualities the $\alpha_{-}$and $\beta_{-}$directions, one finds a new background with the metric

$$
d s^{2}=\frac{1+y_{1}}{2\left(1-y_{1}\right)} d \alpha_{-}^{2}+\frac{1}{2}\left(d \alpha_{-}-2 d \alpha_{+}\right)^{2}+\frac{1+y_{2}}{2\left(1-y_{2}\right)} d \beta_{-}^{2}+\frac{1}{2}\left(d \beta_{-}-2 d \beta_{+}\right)^{2},
$$

but without the $B$ field. Comparison with (2.120) shows that the dual geometry is $[\mathrm{SO}(4) /[\mathrm{SO}(2) \times \mathrm{SO}(2)]] \times \mathrm{U}(1)^{2}$. This agrees with a general statement that gauging of any $\mathrm{SO}(2)$ symmetry is equivalent to a $\mathrm{T}$ duality [61]. By performing only one T-duality in (2.152), one would find $[\mathrm{SO}(4) / \mathrm{SO}(2)] \times \mathrm{U}(1)$.

The map between $\mathrm{SO}(4) / \mathrm{SO}(2)$ and $\mathrm{SO}(4) /[\mathrm{SO}(2) \times \mathrm{SO}(2)]$ cosets is slightly more interesting. The T-duality corresponding to this map is performed along some combination 
of angles appearing in (2.91). Specifically, introducing a new coordinate $\tau=2 \gamma+\alpha$, we can rewrite the $\mathrm{SO}(4) / \mathrm{SO}(2)$ metric $(2.91)$ as: ${ }^{18}$

$$
\begin{aligned}
d s^{2}= & \frac{\left(y_{1} y_{2}-1\right)}{y_{1}+y_{2}-2} d \alpha^{2}+\frac{2\left(y_{1}-y_{2}\right)}{y_{1}+y_{2}-2} d \alpha d \beta-\frac{\left(y_{1}-1\right)\left(y_{2}-1\right)}{y_{1}+y_{2}-2} d \tau^{2} \\
& -\frac{y_{1}+y_{2}+2}{y_{1}+y_{2}-2} d \beta^{2}+\frac{d y_{1}^{2}}{2-2 y_{1}^{2}}+\frac{d y_{2}^{2}}{2-2 y_{2}^{2}} \\
B= & d \tau \wedge\left[d \beta \frac{y_{2}-y_{1}}{y_{2}+y_{1}-2}-d \alpha \frac{\left(y_{1} y_{2}-1\right)}{y_{2}+y_{1}-2}\right], \quad e^{-2 \Phi}=2-y_{1}-y_{2} .
\end{aligned}
$$

T duality along $\tau$ direction removes the $B$-field and makes the dilaton separable:

$$
\begin{aligned}
d s^{2}= & \frac{1-y_{1} y_{2}}{\left(y_{1}-1\right)\left(y_{2}-1\right)}\left[d \alpha^{2}+d \beta^{2}\right]-\frac{2\left(y_{1}-y_{2}\right) d \alpha d \beta}{\left(y_{1}-1\right)\left(y_{2}-1\right)}-\frac{y_{1}+y_{2}-2}{\left(y_{1}-1\right)\left(y_{2}-1\right)} d \tau^{2} \\
& 2 d \tau \frac{\left(y_{1}-y_{2}\right) d \beta-\left(1-y_{1} y_{2}\right) d \alpha}{\left(y_{1}-1\right)\left(y_{2}-1\right)}+\frac{d y_{1}^{2}}{2-2 y_{1}^{2}}+\frac{d y_{2}^{2}}{2-2 y_{2}^{2}} \\
e^{-2 \Phi}= & \left(y_{1}-1\right)\left(y_{2}-1\right) .
\end{aligned}
$$

An additional shift, $\alpha \rightarrow \alpha+\tau$ leads to a simpler metric (2.120) with an additional flat direction $\tau$ :

$$
\begin{aligned}
d s^{2} & =\frac{1-y_{1} y_{2}}{\left(y_{1}-1\right)\left(y_{2}-1\right)}\left[d \alpha^{2}+d \beta^{2}\right]-\frac{2\left(y_{1}-y_{2}\right) d \alpha d \beta}{\left(y_{1}-1\right)\left(y_{2}-1\right)}+d \tau^{2}+\frac{d y_{1}^{2}}{2-2 y_{1}^{2}}+\frac{d y_{2}^{2}}{2-2 y_{2}^{2}} \\
& =\frac{1+y_{2}}{2\left(1-y_{2}\right)}[d \alpha+d \beta]^{2}+\frac{1+y_{1}}{2\left(1-y_{1}\right)}[d \alpha-d \beta]^{2}+d \tau^{2}+\frac{d y_{1}^{2}}{2-2 y_{1}^{2}}+\frac{d y_{2}^{2}}{2-2 y_{2}^{2}} \\
e^{-2 \Phi} & =\left(y_{1}-1\right)\left(y_{2}-1\right) .
\end{aligned}
$$

As expected, this is the $[\mathrm{SU}(2) / \mathrm{U}(1)] \times[\mathrm{SU}(2) / \mathrm{U}(1)] \times \mathrm{U}(1)$ geometry.

Once various $\mathrm{SO}(4) / H$ backgrounds are shown to be related by T-dualities, separation of variables on one of them guarantees separation on another provided that dynamical equations remain invariant. In particular, the scalar equation (2.96) is invariant under a T-duality if and only if $\sigma=1$, so separability of the Helmholtz equation on $\mathrm{SO}(4)$, where the dilaton is trivial, would imply separability on $\mathrm{SO}(4) / H$ only for $\sigma=1$. We saw this explicitly for the $\mathrm{SO}(4) / \mathrm{SO}(2)$ coset in section 2.3. Interestingly, the scalar equation on the $\mathrm{SO}(4) /[\mathrm{SO}(2) \times \mathrm{SO}(2)]$ geometry separates for an arbitrary $\sigma$ (see (2.123)), but such "bonus separation" is not a consequence of T-duality.

To separate the vector equation (2.103), one needs to build special frames associated with the Killing-Yano tensors (see, for example, (2.106)). The behavior of the KillingYano tensors (KYT) under T-duality was studied in [28], where it was shown that while the ordinary KYTs may disappear, the modified KYTs are preserved. Interestingly, it is precisely such modified Killing-Yano tensors, (2.107) and (2.114), that are responsible for separation of the vector equations after T-duality. Therefore, we have demonstrated that separations of the scalar and vector equations on the $\mathrm{SO}(4) / H$ cosets are not accidental, but rather they are guaranteed by the relation between gauging and T-duality [61] and by the transformation of dynamical equations and Killing-Yano tensors under the duality [28].

\footnotetext{
${ }^{18}$ In the subsection we have dropped the factor $k / \pi$.
} 


\section{The SO(5) sigma model}

In this section we will look at separation of variables in the $\mathrm{SO}(5)$ sigma model. Unfortunately the full separation of variables encountered in previous section for $\mathrm{SO}(4)$ and it cosets does not persist for $\mathrm{SO}(5)$, but we find several interesting sectors which admit a partial separation. We begin with reviewing parameterization of $\mathrm{SO}(5)$ and an algebraic construction of the scalar eigenfunctions developed in [5]. In section 3.1 we also present some simple examples of wavefunctions which inspire the analysis in the rest of the discussion of the $\mathrm{SO}(5)$ group. In sections 3.3 and 3.4 we construct two infinite classes of separable eigenfunctions by solving the Helmholtz equation. Each family is parameterized by four discrete quantum numbers. In section 3.5 we use an algebraic procedure to construct additional infinite families of separable solutions which depend on four parameters as well. Finally, in section 3.6 we discuss partial separation for a different parameterization of $\mathrm{SO}(5)$ as well as its extensions to larger groups.

The action of the $\mathrm{SO}(5)$ WZW model,

$$
S=-\frac{k}{2 \pi} \int d^{2} \sigma \eta^{\alpha \beta} \operatorname{tr}\left(g^{-1} \partial_{\alpha} g g^{-1} \partial_{\beta} g\right)+\frac{i k}{6 \pi} \int \operatorname{tr}\left(g^{-1} d g \wedge g^{-1} d g \wedge g^{-1} d g\right)
$$

is invariant under the $\mathrm{SO}(5)_{L} \times \mathrm{SO}(5)_{R}$ global symmetry. Since $\mathrm{SO}(5)$ has rank two, the sigma model (3.1) has $2+2=4$ commuting Killing vectors. It is useful to realize these $\mathrm{U}(1)$ symmetries by simple translations, and this can be accomplished by the following parameterization of the group element $g$ :

$$
g=h\left[\alpha_{L}, \beta_{L}\right]\left[\begin{array}{cc}
I-B_{X} X^{T} X & -B_{X} X^{T} \\
B_{X} X & I-B_{X} X X^{T}
\end{array}\right]\left[\begin{array}{ccc}
I-B_{Y} Y Y^{T} & B_{Y} Y & 0 \\
-Y^{T} B_{Y} & I-Y^{T} B_{Y} Y & 0 \\
0 & 0 & 1
\end{array}\right] h\left[\alpha_{R}, \beta_{R}\right] .
$$

Here vector $X$, scalar $B_{X}$, and matrices $\left(Y, B_{Y}\right)$, are defined by

$$
X=\left(X_{1}, X_{2}, X_{3}, X_{4}\right), \quad B_{X}=\frac{2}{1+X X^{T}} \quad Y=\operatorname{diag}\left(Y_{1}, Y_{2}\right), \quad B_{Y}=\frac{2}{1+Y Y^{T}} .
$$

We also defined $h[\alpha, \beta]$ as a matrix function of two angles:

$$
h[\alpha, \beta]=\left[\begin{array}{ccccc}
c_{\alpha} & s_{\alpha} & 0 & 0 & 0 \\
-s_{\alpha} & c_{\alpha} & 0 & 0 & 0 \\
0 & 0 & c_{\beta} & s_{\beta} & 0 \\
0 & 0 & -s_{\beta} & c_{\beta} & 0 \\
0 & 0 & 0 & 0 & 1
\end{array}\right] .
$$

Note that matrix $h_{L}=h\left[\alpha_{L}, \beta_{L}\right]$ appears in the action (3.1) only in the combination $h_{L}^{-1} d h_{L}$, so coordinates $\left(\alpha_{L}, \beta_{L}\right)$ are cyclic. Similarly, matrix $h_{R}=h\left[\alpha_{R}, \beta_{R}\right]$ appears only in the combination $d h_{R} h_{R}^{-1}$, so coordinates $\left(\alpha_{R}, \beta_{R}\right)$ are cyclic as well. The full metric corresponding to (3.1) is rather complicated, and here we just stress one important 
property, which is easy to verify. If we write $X_{i}=R \mu_{i}$, where three variables $\mu_{i}$ are subject to constraint $\sum \mu_{1}^{2}=1$, then

$$
d s^{2}=\frac{k}{2 \pi}\left[\frac{8 d R^{2}}{\left(1+R^{2}\right)^{2}}+(\text { terms without } d R)\right] .
$$

In other words, the cross terms between $d X_{i}$ and remaining coordinates can be written in terms of $d \mu_{i}$.

In this section we will study the scalar equation

$$
\nabla^{2} \Phi=-\frac{\pi}{k} \Lambda \Phi
$$

in the geometry (3.1)-(3.2). As demonstrated in $[2,3]$, the eigenvalues of this equation can be expressed in terms of the quadratic Casimir of the gauge group, and the $\mathrm{SO}(5)$ case, the result is

$$
\Lambda=l_{1}\left(l_{1}+3\right)+l_{2}\left(l_{2}+1\right), \quad l_{1} \geq l_{2},
$$

where $\left(l_{1}, l_{2}\right)$ are either both integers or both half-integers. Our goal is to construct the corresponding eigenfunctions. In contrast to the situation described in the previous section, equation (3.6) is not fully separable for the SO(5) WZW model, but there are several separable families and they will be described in separate subsections. The simplest family follows from the observation (3.5): if we assume that $\Phi$ is a function of $R$ only, then the equation (3.6) becomes $^{19}$

$$
\frac{\left(1+R^{2}\right)^{4}}{4 R^{3}} \frac{d}{d R}\left[\frac{R^{3}}{\left(1+R^{2}\right)^{2}} \frac{d \Phi}{d R}\right]+\Lambda \Phi=0 .
$$

The normalizable solutions are

$$
\Phi=F\left[-k, 3+k ; 2 ; \frac{1}{1+R^{2}}\right], \quad \Lambda=\frac{(3+2 k)^{2}-9}{4},
$$

where $k$ is a non-negative integer, so we recover $(3.7)$ with $\left(l_{1}, l_{2}\right)=(k, 0)$. In the remaining part of this section we will extend the explicit solution (3.9) to more general families.

\subsection{Eigenfunctions from group theory}

Before analyzing differential equations, it is useful to recall the algebraic construction for the eigenfunctions of the Helmholtz equation (3.6). As demonstrated in $[2,3]$, all such eigenfunctions can be constructed as polynomials in the matrix elements of $g$. Specifically, each eigenvalue (3.7) corresponds to an irreducible representation of SO(5). Such representations are characterized by Young tableaux, which in turn specify representations of the permutation group $S_{5}$. Then the wavefunction $\Phi$ is written as the sum over relevant permutations $P[5]$

$$
\Phi=\sum_{P}(-1)^{\sigma(P)} g_{i_{1} j_{P[1]}} \ldots g_{i_{L} j_{P[L]}}-(\text { traces }) .
$$

\footnotetext{
${ }^{19}$ We used the expression for the determinant of the metric.
} 
The wavefunction is fully specified by the set of $2 L$ indices $\left(i_{1}, \ldots, i_{L}, j_{1}, \ldots j_{L}\right)$ and the signatures $\sigma(P)$ associated with the Young tableau. In this subsection we will present several examples of eigenfunctions (3.10) for representation of $\mathrm{SO}(5)$ with small $L$, and in the subsequent subsections the patterns observed in these examples will be used to construct infinite separable families.

The first set of states corresponds to the Young tableau with one box. The eigenvalue is

$$
\Lambda=4
$$

and the eigenfunctions are arbitrary linear combinations of the matrix elements $g_{i j}$. To make the $[\mathrm{U}(1)]^{4}$ symmetries explicit, we focus on the combinations which have specific charges under these transformations. There are 25 states in total. One of them is neutral, and it corresponds to (3.9) with $k=1$ :

$$
\frac{1-R^{2}}{1+R^{2}}
$$

This is the only state in the $k=1$ representation that does not have angular or $X_{a}$ dependence.

To write the remaining states in the $k=1$ representation, it is convenient to introduce three combinations of the coordinates $\left(R, Y_{1}, Y_{2}\right)$,

$$
D=\frac{\left(1+Y_{1}^{2}\right)\left(1+Y_{2}^{2}\right)\left(1+R^{2}\right)}{1-Y_{1}^{2} Y_{2}^{2}}, \quad y_{+}=\frac{Y_{1}+Y_{2}}{1-Y_{1} Y_{2}}, \quad y_{-}=\frac{Y_{1}-Y_{2}}{1+Y_{1} Y_{2}}
$$

as well as six complex combinations of $X_{a}$,

$$
\begin{aligned}
z_{1} & =X_{1}+i X_{2}, \quad z_{2}=X_{3}+i X_{4}, \\
Z_{1+} & =z_{1}-Y_{+} z_{2}, \quad Z_{2+}=z_{2}+y_{+} z_{1}, \quad Z_{1-}=z_{1}-y_{-} \bar{z}_{2}, \quad Z_{2-}=z_{2}+y_{-} \bar{z}_{1} .
\end{aligned}
$$

Then we find that the 25 states in the $k=1$ representation can be divided in four groups:

1. One state (3.12) without angular or $X_{a}$ dependence.

2. Eight states are linear in $X_{a}$. They are given by

$$
\frac{e^{-2 i \alpha_{L}} z_{1}}{1+R^{2}}, \quad \frac{e^{-2 i \beta_{L}} z_{2}}{1+R^{2}}, \quad \frac{e^{2 i \alpha_{R}}\left(Z_{1+}-Y_{-} \bar{Z}_{2+}\right)}{D}, \frac{e^{2 i \beta_{R}}\left(Z_{2+}+Y_{-} \bar{Z}_{1+}\right)}{D},
$$

and their complex conjugates.

3. Eight states charged under $\mathrm{U}(1)_{\alpha_{L}}$ symmetry are given by

$$
\begin{array}{ll}
e^{-2 i\left(\alpha_{L}+\alpha_{R}\right)} \frac{\left(1+Z_{2+} \bar{Z}_{2-}\right)}{D}, & e^{-2 i\left(\alpha_{L}+\beta_{R}\right)} \frac{\left(Z_{1+} \bar{Z}_{2-}-Y_{+}\right)}{D}, \\
e^{2 i\left(\alpha_{R}-\alpha_{L}\right)} \frac{\left(Y_{+} Y_{-}+Z_{1+} Z_{1-}\right)}{D}, & e^{-2 i\left(\alpha_{L}-\beta_{R}\right)} \frac{\left(Z_{1-} Z_{2+}-Y_{-}\right)}{D}
\end{array}
$$

and their complex conjugates. 
4. Eight states charged under $\mathrm{U}(1)_{\beta_{L}}$ symmetry can be obtained from (3.16) and their complex conjugates by the replacements

$$
\alpha_{L} \rightarrow \beta_{L}, \quad \alpha_{R} \rightarrow \beta_{R}, \quad Z_{1 \pm} \rightarrow Z_{2 \pm}, \quad Z_{2 \pm} \rightarrow-Z_{1 \pm}
$$

The extensions of these groups to general families will be discussed in section 3.4. We conclude this subsection by listing some solutions corresponding to antisymmetric representation characterized by a Young tableau with two boxes. The eigenvalue $\Lambda=4$ has degeneracy 100, and the wavefunctions are specified by two antisymmetric pairs of indices, $(i, j)$ and $(k, l) .{ }^{20} \mathrm{Up}$ to a normalization factor, the wavefunctions are given by

$$
\Phi_{i j ; k l}=g_{i k} g_{j l}-g_{i l} g_{j k} .
$$

As we saw already in the case of the fundamental representation, it is convenient to introduce complex coordinates (3.14), so we will use the values $\left(z_{1}, \bar{z}_{1}, z_{2}, \bar{z}_{2}, 5\right)$ for indices $(i, j, k, l)$ as well. For example,

$$
\Phi_{z_{1} j ; k l}=\Phi_{1 j ; k l}+i \Phi_{2 j ; k l}, \quad \Phi_{z_{2} j ; k l}=\Phi_{1 j ; k l}-i \Phi_{2 j ; k l} .
$$

Substituting the explicit expressions for the matrix elements of $g$, we observe that the following combinations, as well as their complex conjugates, depend on $\left(z_{1}, \bar{z}_{1}, z_{2}, \bar{z}_{2}\right)$ only through $R^{2}$ :

$$
\begin{array}{ll}
\Phi_{z_{1} z_{2} ; z_{1} z_{2}}=\frac{4 E_{1,1,1,1}\left(1+Y_{1} Y_{2}\right)}{D\left(1-Y_{1} Y_{2}\right)}, & \Phi_{z_{1} z_{2} ; \bar{z}_{1} \bar{z}_{2}}=\frac{4 E_{1,1,-1,-1}\left(Y_{1}-Y_{2}\right)^{2}}{D\left[1-\left(Y_{1} Y_{2}\right)^{2}\right]} \\
\Phi_{z_{1} \bar{z}_{2} ; z_{1} \bar{z}_{2}}=\frac{4 E_{1,-1,1,-1}\left(1-Y_{1} Y_{2}\right)}{D\left(1+Y_{1} Y_{2}\right)}, & \Phi_{z_{1} \bar{z}_{2} ; \bar{z}_{1} z_{2}}=\frac{4 E_{1,-1,-1,1}\left(Y_{1}+Y_{2}\right)^{2}}{D\left[1-\left(Y_{1} Y_{2}\right)^{2}\right]} .
\end{array}
$$

Here we introduced a convenient shorthand notation

$$
E_{a, b, c, d}=e^{-2 i\left(a \alpha_{L}+b \beta_{L}+c \alpha_{R}+d \beta_{R}\right)} .
$$

Note that wavefunctions (3.20) have a separable structure

$$
\Phi=e^{-2 i\left(a \alpha_{L}+b \beta_{L}+c \alpha_{R}+d \beta_{R}\right)} f(R) g\left(Y_{1}, Y_{2}\right) .
$$

In the next two subsections we will construct the most general function of the form (3.22) that solves the scalar equation (3.6). In sections 3.4 and 3.5 extensions to several classes of $z$-dependent solutions will be discussed as well, and they will contain the states (3.15) and (3.16) as special cases.

\footnotetext{
${ }^{20}$ Recall that $g$ is a $5 \times 5$ matrix, so the indices $(i, j, k, l)$ range from one to five. Then the antisymmetric combinations, $(i, j)$ and $(k, l)$, can take 10 possible values each. This explains the 100 -fold degeneracy of the eigenvalue $\Lambda=6$.
} 


\subsection{Factorization of the $R$ dependence}

Before finding the most general solutions of the form (3.22), it is instructive to start with a specific solution, such as one of the functions listed in (3.20), and explore the possibility of changing function $f(R)$ while keeping $g\left(Y_{1}, Y_{2}\right)$ and constants $(a, b, c, d)$ fixed. This subsection is dedicated to the discussion of such " $R$-dressing", and our starting point will be slightly more general than (3.22).

Let us assume that the Helmholtz equation (3.6) has a solution of the form

$$
\Phi_{\Lambda_{0}}=\frac{R^{p}}{\left(1+R^{2}\right)^{q}} \tilde{\Phi}\left(\gamma_{i}, Y_{a}, \frac{X_{j}}{R}\right), \quad \gamma_{i}=\left\{\alpha_{L}, \beta_{L}, \alpha_{R}, \beta_{R}\right\}
$$

In particular, wavefunctions (3.22) and (3.15) fit this pattern. We will now demonstrate that equation (3.6) admits a family of normalizable solutions which are obtained by "dressing" solutions (3.23) by some specific function of the radial coordinate:

$$
\Phi_{\Lambda_{k}}^{(k)}=\frac{R^{p}}{\left(1+R^{2}\right)^{q}} f_{k, p, q}(R) \tilde{\Phi}\left(\gamma_{i}, Y_{a}, \frac{X_{j}}{R}\right), \quad \Lambda_{k}=\Lambda_{0}+(2 k+2 q+3)^{2}-(2 q+3)^{2} .
$$

The "dressed" solution depends on an integer parameter $k$. To prove (3.24), we recall that the metric has the form (3.5), where "terms without $d R$ " contain $\left(d \gamma_{i}, d Y_{a}, d \mu_{j}\right)$, where $\mu_{j}$ are three angles from a constrained set of four parameters:

$$
X_{j}=R \mu_{j}, \quad \sum \mu_{j}^{2}=1 .
$$

The differential equation (3.6) has the form

$$
\frac{\left(1+R^{2}\right)^{4}}{4 R^{3}} \frac{\partial}{\partial R}\left[\frac{R^{3}}{\left(1+R^{2}\right)^{2}} \frac{\partial \Phi}{\partial R}\right]+\tilde{\nabla}^{2} \Phi+\Lambda \Phi=0,
$$

where $\tilde{\nabla}^{2}$ has a complicated $R$-dependence, but no $R$-derivatives. Writing equation (3.26) for two wavefunctions, (3.24) and (3.23), and combining the results to eliminate the terms with $\tilde{\nabla}^{2}$, we find

$$
\begin{aligned}
& \frac{\left(1+R^{2}\right)^{4}}{4 R^{3}} \frac{\partial}{\partial R}\left[\frac{R^{3}}{\left(1+R^{2}\right)^{2}} \frac{\partial \Phi_{\Lambda_{k}}^{(k)}}{\partial R}\right]+\left(\Lambda_{k}-\Lambda_{0}\right) \Phi_{\Lambda_{k}}^{(k)} \\
& =\frac{\left(1+R^{2}\right)^{4+q}}{4 R^{3+p}} \frac{\partial}{\partial R}\left[\frac{R^{3}}{\left(1+R^{2}\right)^{2}} \frac{d}{d R} \frac{R^{p}}{\left(1+R^{2}\right)^{q}}\right] \Phi_{\Lambda_{k}}^{(k)} .
\end{aligned}
$$

To simplify this equation, we define a new function $h$ by

$$
h\left[\frac{R^{2}}{1+R^{2}}\right] \equiv f_{k, p, q}(R) .
$$

Then equation (3.27) becomes

$$
x(1-x) h^{\prime \prime}+[2+p-2(2+q) x] h^{\prime}+\left(\Lambda_{k}-\Lambda_{0}\right) h=0,
$$


and the solution regular at $R=0$ can be expressed in terms of the hypergeometric function:

$$
\begin{aligned}
f_{k, p, q}(R) & =F\left[-k, 3+k+2 q ; 2+p ; \frac{R^{2}}{1+R^{2}}\right], \\
\text { where } \Lambda_{k} & =\Lambda_{0}+\frac{(2 k+2 q+3)^{2}-(2 q+3)^{2}}{4} .
\end{aligned}
$$

Normalizability at large values of $R$ requires $k$ to be a non-negative integer.

To summarize, we have demonstrated that every wavefunction of the form (3.23) gives rise to a one-parametric family of normalizable solutions of the Helmholtz equation (3.6). The wavefunctions are

$$
\Phi_{\Lambda_{k}}^{(k)}=\frac{R^{p}}{\left(1+R^{2}\right)^{q}} F\left[-k, 3+k+2 q ; 2+p ; \frac{R^{2}}{1+R^{2}}\right] \tilde{\Phi}\left(\gamma_{i}, Y_{a}, \frac{X_{j}}{R}\right),
$$

and the eigenvalues are

$$
\Lambda_{k}=\Lambda_{0}+\frac{(2 k+2 q+3)^{2}-(2 q+3)^{2}}{4} .
$$

Normalizabilty requires $k$ to be a non-negative integer. An alternative form of $(3.31),{ }^{21}$

$$
\Phi_{\Lambda_{k}}^{(k)}=\frac{R^{p}}{\left(1+R^{2}\right)^{q}} F\left[-k, 3+k+2 q ; 2+2 q-p ; \frac{1}{1+R^{2}}\right] \tilde{\Phi}\left(\gamma_{i}, Y_{a}, \frac{X_{j}}{R}\right),
$$

may be useful as well. In particular, the solution (3.9) is recovered by choosing the trivial function $\tilde{\Phi}$ and $p=q=0$.

We conclude this subsection with presenting an example of the dressing (3.31). Observing that the wavefunctions $(3.20)$ have $(p, q)=(0,1)$, we can dress the first wavefunction as

$$
\Phi_{z_{1} z_{2} ; z_{1} z_{2}}^{(k)}=\frac{4 E_{1,1,1,1}\left(1+Y_{1} Y_{2}\right)}{D\left(1-Y_{1} Y_{2}\right)} F\left[-k, 5+k ; 2 ; \frac{R^{2}}{1+R^{2}}\right], \Lambda_{k}=\Lambda_{0}+\frac{(2 k+5)^{2}-25}{4} .
$$

The remaining wavefunction from (3.20), as well as examples from (3.15) can be dressed in the same way.

\subsection{Separable $X$-independent solutions}

In this subsection we will generalize the solutions (3.20) to wavefunctions which have the form (3.22). For fixed function $g$ and parameters $(a, b, c, d)$, solution (3.22) covers a oneparameter family of "dressed" wavefunctions analyzed in the previous subsection. To avoid unnecessary complications associated with $f(R)$, here we will focus on the "seed solutions" (3.23):

$$
\Phi=e^{2 i\left[n_{1} \alpha_{L}+n_{2} \beta_{L}+n_{3} \alpha_{R}+n_{4} \beta_{R}\right]} \frac{g\left[Y_{1}, Y_{2}\right]}{\left(1+R^{2}\right)^{q}},
$$

\footnotetext{
${ }^{21}$ We dropped a constant multiplicative factor.
} 
and the "dressing" will be added in the end. In contrast to (3.23), equation (3.35) lists the $[\mathrm{U}(1)]^{4}$ charges explicitly. Also, since we are looking for solutions independent of $\mu_{j}=X_{j} / R$, the parameter $p$ in the seed solution (3.23) vanishes. Substitution of the ansatz (3.35) into the Helmholtz equation (3.6) leads to a complicated overdetermined system of equations for the function $g\left[Y_{1}, Y_{2}\right] \cdot{ }^{22}$ The explicit form of these equations is not very illuminating, so we will present only the logic for solving them.

(i) Once the ansatz (3.35) is substituted into the equation (3.6), one finds an equation that contains various functions of $\left(X_{1}, X_{2}, X_{3}, X_{4}\right)$ and $R$. Expressing $X_{1}$ in terms of the remaining variables, one finds a system with independent $\left(R, X_{2}, X_{3}, X_{4}\right)$. In particular, the coefficient in front of the product $\left(X_{2} X_{4}\right)$ contains a polynomial in $\left(Y_{1}, Y_{2}\right)$ which must vanish. This happens if and only if

$$
n_{4}=\frac{n_{2} n_{3}}{n_{1}} \text { and } n_{2}= \pm n_{1} .
$$

This leads to two branches for the solution (3.35).

(ii) Focusing on the $n_{1}=n_{2}$ branch, and requiring the coefficient of (3.6) in front of $X_{2}$ to vanish, we find a first order equation for the function $g$ :

$$
\left(1+Y_{2}^{2}\right) \partial_{Y_{2}} g+\left(1+Y_{1}^{2}\right) \partial_{Y_{1}} g=0
$$

This reduces $g\left[Y_{1}, Y_{2}\right]$ to a function of one variable:

$$
n_{2}=n_{1} \quad \Rightarrow \quad g=f\left[\frac{Y_{1}-Y_{2}}{1+Y_{1} Y_{2}}\right] .
$$

Similarly, the $n_{2}=-n_{1}$ branch gives

$$
n_{2}=-n_{1} \Rightarrow\left(1+Y_{1}^{2}\right) \partial_{Y_{1}} g-\left(1+Y_{2}^{2}\right) \partial_{Y_{2}} g=0 \Rightarrow g=f\left[\frac{Y_{1}+Y_{2}}{1-Y_{1} Y_{2}}\right]
$$

(iii) Substitution of (3.38) or (3.39) into (3.6) reduces the Helmholtz equation to a single ODE for the unknown function $f$, and the resulting normalizable wavefunctions $\Phi$ are given by (3.40).

After this summary of the derivation we present the final result. The two branches of the solution (3.35) can be written as

$$
\begin{aligned}
& \Phi=\frac{e^{2 i\left[n_{1}\left(\alpha_{L}+\beta_{L}\right)+n_{3}\left(\alpha_{R}+\beta_{R}\right)\right]}}{\left(1+R^{2}\right)^{q}} \frac{\left(1+y_{-}^{2}\right)^{q+1}}{y_{-}^{n_{1}-n_{3}}} F\left[q+1-n_{1}, q+1+n_{3} ; 1-n_{1}+n_{3} ;-y_{-}^{2}\right] \\
& \Phi=\frac{e^{2 i\left[n_{1}\left(\alpha_{L}-\beta_{L}\right)+n_{3}\left(\alpha_{R}-\beta_{R}\right)\right]}}{\left(1+R^{2}\right)^{q}} \frac{\left(1+y_{+}^{2}\right)^{q+1}}{y_{+}^{n_{1}-n_{3}}} F\left[q+1-n_{1}, q+1+n_{3} ; 1-n_{1}+n_{3} ;-y_{+}^{2}\right],
\end{aligned}
$$

\footnotetext{
${ }^{22}$ Specifically, variables $\left(X_{1}, X_{2}, X_{3}, X_{4}\right)$ appear in the equation (3.6) in various combinations, not only as $R^{2}$. This leads to a system of PDEs for one function $g\left[Y_{1}, Y_{2}\right]$.
} 
and complex conjugates of these expressions. Here we used the convenient variables $y_{ \pm}$ defined in (3.13):

$$
y_{ \pm}=\frac{Y_{1} \pm Y_{2}}{1 \mp Y_{1} Y_{2}} .
$$

Interestingly, the eigenvalues corresponding to functions (3.40) depends only on $q$ :

$$
\Lambda=2 q(2+q)
$$

and parameters $\left(n_{1}, n_{3}\right)$ enter only through the constraints

$$
\max \left(n_{1}, n_{3}\right) \leq q, \quad \text { integer } \quad\left(n_{1}, n_{2}, q\right) .
$$

Solutions (3.40) can be dressed with functions of $R$ according to (3.31)-(3.32):

$$
\begin{aligned}
\frac{1}{\left(1+R^{2}\right)^{q}} & \rightarrow \frac{1}{\left(1+R^{2}\right)^{q}} F\left[-k, k+2 q+3 ; 2+2 q ; \frac{1}{1+R^{2}}\right], \\
\Lambda & =2 q(2+q)+(2 k+2 q+3)^{2}-(2 q+3)^{2} .
\end{aligned}
$$

In two special cases, $n_{1}=n_{3}=q$ and $n_{1}=-n_{3}=-q$, the $y$-dependent parts of $(3.40)$ simplify to For $n_{1}=n_{3}=\nu$, the $z$-dependent parts simplify to

$$
\frac{1}{\left(1+y_{ \pm}^{2}\right)^{q}}=\left[\frac{\left(1 \pm Y_{1} Y_{2}\right)^{2}}{\left(1+Y_{1}^{2}\right)\left(1+Y_{2}^{2}\right)}\right]^{q} \text { and }\left[\frac{y_{ \pm}^{2}}{1+y_{ \pm}^{2}}\right]^{q}=\left[\frac{\left(Y_{1} \pm Y_{2}\right)^{2}}{\left(1+Y_{1}^{2}\right)\left(1+Y_{2}^{2}\right)}\right]^{q}
$$

leading to pure powers of the expressions (3.20).

To summarize, we have demonstrated that the $X$-independent ansatz (3.35) introduces constraints (3.36) on the $[\mathrm{U}(1)]^{4}$ charges and reduces $g\left[Y_{1}, Y_{2}\right]$ to a function of one variable. This implies, that the solution (3.35) depends on three parameters: two combinations of $\left(n_{1}, n_{2}, n_{3}, n_{4}\right)$ which are not eliminated by the constraint (3.36), and an additional integer coming from the solutions of the ODE for the function $g$. Dressing the solutions (3.40) with a function of $R$ introduces the fourth parameter. Since the most general solution of the Helmholtz equation (3.6) is expected to depend on 10 parameters, clearly the wavefunctions (3.35) form a very small subset. Unfortunately, the nice separability encountered in (3.35) does not persist for the $X$-dependent functions, but several infinite families of wavefunctions can be constructed, and they will be discussed in the next subsection.

\subsection{Solutions linear in $X$ coordinates}

In the previous subsection we have constructed the most general $X$-independent solution of the Helmholtz equation (3.6). Unfortunately, explicit closed-form expressions for all $X$ dependent eigenfunctions are unlikely to exist. ${ }^{23}$ Nevertheless in this subsection we construct several infinite families of $X$-dependent eigenfunctions, and these results can be viewed as a complement of the algebraic procedure (3.10), which is practical only for representations with a small number of boxes in the Young diagrams.

\footnotetext{
${ }^{23}$ Procedure (3.10) allows to construct all such functions algorithmically, but the combinatorics becomes complicated.
} 
Let us look at wavefunctions which are linear in $\left(X_{1}, X_{2}, X_{3}, X_{4}\right)$. The explicit examples (3.15) suggest that it might be useful to write the solutions in terms of complex variables $\left(z_{1}, \bar{z}_{1}, z_{2}, \bar{z}_{2}\right)$. Let us impose an ansatz

$$
\Phi=\frac{e^{2 i\left[n_{1} \alpha_{L}+n_{2} \beta_{L}+n_{3} \alpha_{R}+n_{4} \beta_{R}\right]}}{\left(1+R^{2}\right)^{q}}\left[z_{1} g_{1}\left(Y_{1}, Y_{2}\right)+\bar{z}_{1} g_{2}\left(Y_{1}, Y_{2}\right)+z_{2} g_{3}\left(Y_{1}, Y_{2}\right)+\bar{z}_{2} g_{4}\left(Y_{1}, Y_{2}\right)\right] .
$$

Substituting this function into the equation (3.6), and requiring the coefficients in front of eight combinations $\left(X_{1}^{2} X_{2}, X_{1}^{3}, X_{2}^{2} X_{1}, X_{2}^{3}, X_{3}^{2} X_{4}, X_{3}^{3}, X_{4}^{2} X_{3}, X_{4}^{3}\right)$, to vanish, we can algebraically solve the resulting equations for the eight second derivatives

$$
\partial_{Y_{1}}^{2} g_{i}\left(Y_{1}, Y_{2}\right), \quad \partial_{Y_{2}}^{2} g_{i}\left(Y_{1}, Y_{2}\right)
$$

Substituting the result back to (3.6), we observe that the coefficients in front of $\left(X_{1}^{2} X_{3}\right.$, $\left.X_{2}^{2} X_{3}, X_{3}^{2} X_{1}, X_{4}^{2} X_{2}\right)$ contain only functions $g_{i}$, but not their derivatives. Requirement of having non-trivial solutions implies that the determinant of the characteristic matrix has to vanish. This condition leads to only eight possibilities:

$$
\begin{array}{ll}
n_{2}=n_{1} \pm 1, & n_{3}=n_{4} \\
n_{2}=-n_{1} \pm 1, & n_{3}=-n_{4} \\
n_{4}=n_{3} \pm 1, & n_{1}=n_{2} \\
n_{4}=-n_{3} \pm 1, & n_{1}=-n_{2} .
\end{array}
$$

Some of the resulting solutions can be obtained from the others by applying discrete symmetries of the metric. First, by taking a complex conjugate of the solution, if necessary, we can focus only on " -1 " option instead of \pm 1 . Furthermore, the first two options in (3.47), are related by changing the signs of $\left(\alpha_{L}, \alpha_{R}\right)$. While such change by itself is not a symmetry of the metric, it is a part of a larger one:

$$
\left(\alpha_{L}, \alpha_{R}, Y_{1}, X_{1}\right) \rightarrow-\left(\alpha_{L}, \alpha_{R}, Y_{1}, X_{1}\right) .
$$

This symmetry also interchanges the last two options in (3.47). Therefore, there are two genuinely distinct possibilities:

$$
n_{2}=n_{1}-1, \quad n_{3}=n_{4} \quad \text { and } \quad n_{4}=n_{3}-1, \quad n_{1}=n_{2} .
$$

If one of these constraints is imposed, some of the algebraic equations for $g_{i}$ can be solved, and the results are

$$
\begin{array}{ll}
n_{2}=n_{1}-1, \quad n_{3}=n_{4}: \quad \Psi=\frac{E_{n_{1}, n_{2}, n_{3}, n_{4}}}{\left(1+R^{2}\right)^{q}}\left[g_{1}\left(Y_{1}, Y_{2}\right) \bar{z}_{1}+g_{2}\left(Y_{1}, Y_{2}\right) z_{2}\right], \\
n_{1}=n_{2}, \quad n_{4}=n_{3}-1: \quad \Psi=\frac{E_{n_{1}, n_{2}, n_{3}, n_{4}}}{\left(1+R^{2}\right)^{q}}\left[\left\{\bar{z}_{2}+\frac{Y_{1}+Y_{2}}{1-Y_{1} Y_{2}} \bar{z}_{1}\right\} g_{4}+\left\{z_{2}+\frac{Y_{1} Y_{2}-1}{Y_{1}+Y_{2}} z_{1}\right\} g_{3}\right] .
\end{array}
$$

The differential equations for these two ansatze are analyzed in the appendix C.1, and they lead to the following solutions. 
- For the first option in (3.50), functions $g_{1}$ and $g_{2}$ can depend on the $\left(Y_{1}, Y_{2}\right)$ coordinates only through the combination $y_{-}$defined in (3.13):

$$
g_{1}\left[Y_{1}, Y_{2}\right]=h_{1}\left[y_{-}\right], \quad g_{2}\left[Y_{1}, Y_{2}\right]=h_{2}\left[y_{-}\right], \quad y_{-}=\frac{Y_{1}-Y_{2}}{1+Y_{1} Y_{2}} .
$$

Function $h_{1}$ and $h_{2}$ satisfy an overdetermined system of ordinary differential equations, and one of the consistency conditions implies that

$$
h_{1}(w)=\frac{1}{2 w \sigma}\left[-w\left(1+w^{2}\right) h_{2}^{\prime}+\left[n_{3}\left(1+w^{2}\right)-n_{1}\left(1-w^{2}\right)\right] h_{2}\right]
$$

with some constant $\sigma$. The remaining equations lead to the expressions for $(\Lambda, \sigma)$ in terms of the parameters $\left(q, n_{1}, n_{3}\right)$ of the ansatz (3.50), and all regular solutions can be divided into two branches:

(a): $\quad \Lambda=2 q(q+2), \sigma=n_{1}+q$,

$$
\begin{aligned}
& n_{1}>n_{3}-1 \Rightarrow h_{2}[w]=\frac{w^{n_{1}-n_{3}}}{\left(1+w^{2}\right)^{q}} F\left[n_{1}-q,-n_{3}-q ; n_{1}+1-n_{3} ;-w^{2}\right], \\
& n_{3}>n_{1}-1 \Rightarrow h_{2}[w]=\frac{w^{n_{3}-n_{1}}}{\left(1+w^{2}\right)^{q}} F\left[n_{3}-q,-n_{1}-q ; n_{3}+1-n_{1} ;-w^{2}\right] ;
\end{aligned}
$$

(b): $\quad \Lambda=2 q(q+1), \sigma=n_{1}-q$,

$$
\begin{aligned}
& n_{1}>n_{3}-1 \Rightarrow h_{2}[w]=\frac{w^{n_{1}-n_{3}}}{\left(1+w^{2}\right)^{q-1}} F\left[1+n_{1}-q, 1-n_{3}-q ; n_{1}+1-n_{3} ;-w^{2}\right], \\
& n_{3}>n_{1}-1 \Rightarrow h_{2}[w]=\frac{w^{n_{3}-n_{1}}}{\left(1+w^{2}\right)^{q-1}} F\left[1+n_{3}-q, 1-n_{1}-q ; n_{3}+1-n_{1} ;-w^{2}\right] .
\end{aligned}
$$

As in the $X$-independent case (3.40), (3.42), the eigenvalue $\Lambda$ depends only on $q$, and the $[\mathrm{U}(1)]^{4}$ charges enter only through the regularity bounds: the first arguments of the hypergeometric functions appearing in (3.53) must be non-positive integers.

- For the second option in (3.50), functions $g_{3}$ and $g_{4}$ can depend on the $\left(Y_{1}, Y_{2}\right)$ coordinates only through the combination $y_{-}$defined in (3.13), up to a fixed prefactor. Specifically, the second line in (3.50) must have the form

$$
\begin{aligned}
n_{1} & =n_{2}, n_{4}=n_{3}-1: \\
\Psi & =\frac{E_{n_{1}, n_{2}, n_{3}, n_{4}}}{\left(1+R^{2}\right)^{q}} \frac{Y_{1}+Y_{2}}{\sqrt{1+Y_{2}^{2}} \sqrt{1+Y_{1}^{2}}}\left[\left\{\bar{z}_{1}+\frac{1}{y_{+}} \bar{z}_{2}\right\} \hat{g}_{4}\left[y_{-}\right]+\left\{z_{2}-\frac{1}{y_{+}} z_{1}\right\} \hat{g}_{3}\left[y_{-}\right]\right] .
\end{aligned}
$$

Functions $\hat{g}_{3}$ and $\hat{g}_{4}$ satisfy an overdetermined system of ODEs, and one of the consistency conditions gives

$$
\hat{g}_{3}=-y \hat{g}_{4}+h, \quad \hat{g}_{4}=\frac{\left[1+2\left(\Lambda-3 q-2 q^{2}\right) y^{2}+\left(n_{1}-n_{3}\right)\left(1+y^{2}\right)\right] h+y\left(1+y^{2}\right) h^{\prime}}{2\left(1-n_{3}+\Lambda-3 q-2 q^{2}\right) y\left(1+y^{2}\right)} .
$$

The remaining equations lead to two branches for function $h$, the counterparts of (3.53) for the present case: 
(a): $\quad \Lambda=2 q(q+2)$

$$
\begin{gathered}
n_{1}>n_{3}-2 \Rightarrow h[w]=\frac{w^{n_{1}-n_{3}+1}}{\left(1+w^{2}\right)^{q-\frac{1}{2}}} F\left[n_{1}-q, 1-n_{3}-q ; 2+n_{1}-n_{3} ;-w^{2}\right] \\
n_{3}>n_{1} \Rightarrow h[w]=\frac{w^{n_{3}-n_{1}-1}}{\left(1+w^{2}\right)^{q-\frac{1}{2}}} F\left[n_{3}-q-1,-n_{1}-q ; n_{3}-n_{1} ;-w^{2}\right]
\end{gathered}
$$

(b): $\quad \Lambda=2 q(q+1)$

$$
\begin{aligned}
n_{1}>n_{3}-2 & \Rightarrow h[w]=\frac{w^{1+n_{1}-n_{3}}}{\left(1+w^{2}\right)^{q-\frac{3}{2}}} F\left[1+n_{1}-q, 2-n_{3}-q ; 2+n_{1}-n_{3} ;-w^{2}\right], \\
n_{3}>n_{1} & \Rightarrow h[w]=\frac{w^{n_{3}-n_{1}-1}}{\left(1+w^{2}\right)^{q-\frac{3}{2}}} F\left[n_{3}-q, 1-n_{1}-q ; n_{3}-n_{1} ;-w^{2}\right] .
\end{aligned}
$$

Once again, the eigenvalue $\Lambda$ depends only on $q$, and the $[\mathrm{U}(1)]^{4}$ charges enter only through the regularity bounds: the first arguments of the hypergeometric functions appearing in (3.56) must be non-positive integers.

To summarize, we have constructed all wavefunctions which have the form (3.45). Apart from the $R$-dependence these solutions are linear in $\left(X_{1}, X_{2}, X_{3}, X_{4}\right)$ coordinates. We have shown that there are only eight possibilities (3.47) for the $[\mathrm{U}(1)]^{4}$ charges, and for each of these options the final answers are specified by three quantum numbers $\left(n_{1}, n_{3}, q\right)$. The solutions can be divided into several groups:

(i) For $n_{2}=n_{1}-1, n_{4}=n_{3}$, the wavefunctions are given by

$$
\Psi=\frac{E_{n_{1}, n_{1}-1, n_{3}, n_{3}}}{\left(1+R^{2}\right)^{q}}\left[h_{1}\left[y_{-}\right] \bar{z}_{1}+h_{2}\left[y_{-}\right] z_{2}\right]
$$

with functions $h_{1}$ and $h_{2}$ given by (3.52) and (3.53).

(ii) The wavefunctions with $\left(n_{2}, n_{4}\right)=\left(-n_{1}-1,-n_{3}\right)$ are obtained by applying the map (3.48) to the solution (3.57). The result reads

$$
\Psi=\frac{E_{-n_{1}, n_{1}-1,-n_{3}, n_{3}}}{\left(1+R^{2}\right)^{q}}\left[-h_{1}\left[-y_{+}\right] z_{1}+h_{2}\left[-y_{+}\right] z_{2}\right] .
$$

(iii) The solutions with $\left(n_{2}, n_{4}\right)=\left( \pm n_{1}+1, \pm n_{3}\right)$ are constructed by taking complex conjugates of (3.57) and (3.58). The four branches covered by the items (i)-(iii) have the general structure similar to (3.57).

(iv) The solution with $\left(n_{2}, n_{4}\right)=\left(n_{1}, n_{3}-1\right)$ has the form (3.54) with various functions given by (3.55) and (3.56).

(v) The wavefunctions with $\left(n_{2}, n_{4}\right)=\left(-n_{1},-n_{3}-1\right)$ are obtained by applying the transformation (3.48) to equations (3.54), (3.55), and (3.56). 
(vi) The solutions with $\left(n_{2}, n_{4}\right)=\left( \pm n_{1}, \pm n_{3}+1\right)$ are obtained by taking complex conjugates of the wavefunctions from the items (iv) and (v). All four solutions covered by the items (iv)-(vi) have the general structure of the state (3.54).

Each of the eight branches discussed in this subsection has wavefunctions which are specified by three integer parameters $\left(n_{1}, n_{3}, q\right)$. The fourth quantum can be added by dressing the solutions using (3.31) and (3.32) with $p=1$. Therefore, we have constructed eight fourparameter branches of scalar wavefunctions with linear dependence on individual $X_{i}$ and a complicated dependence on $R$.

\subsection{Separable states in symmetric representations}

In the last few subsections we have constructed several infinite families of scalar eigenfunctions by solving the differential equation (3.6). Alternatively, one can use the algebraic method (3.10), but unfortunately it involves combinatorics which becomes very complicated as the size of a representation grows. Nevertheless, the construction (3.10) can be used to find some infinite families of wavefunctions in a closed form, and in this subsection we will do so for the fully symmetric representations of $\mathrm{SO}(5)$. We will begin with re-casing the family (3.9) as a summation (3.10) for the symmetric representations, and then we will extend this construction to more general wavefunctions in such representation.

While it is very easy to find the family (3.9) using differential equations (one just needs to use the general structure (3.5) of the metric), it is instructive to recover these solutions from the group theoretic construction (3.10). Since solutions (3.9) are neutral under $[\mathrm{U}(1)]^{2}$, and they depend only on $R$, but not on the individual coordinates $\left(X_{1}, X_{2}, X_{3}, X_{4}, Y_{1}, Y_{2}\right)$, it is clear that the wavefunctions are built only from $g_{55}$ in the parameterization (3.2). With only one available matrix element, the wavefunction (3.10) vanishes unless the representation is fully symmetric, and in the latter case one has

$$
\Phi=g_{55} \ldots g_{55}-(\text { traces }) .
$$

After recovering the family (3.9) from the last equation, we will analyze more general states constructed from products of $g_{a 5}$ : once again, since all ingredients have the same second index, only symmetric representations are allowed, and the expression (3.10) reduces to

$$
\Phi=g_{a_{1} 5} \ldots g_{a_{k} 5}-(\text { traces })
$$

The structure of traces in (3.59) and (3.60) will be specified below.

We begin with analyzing the wavefunction (3.59). In general, the state in a symmetric representation is ${ }^{24}$

$$
\Phi=g_{a_{1} b_{1}} \ldots g_{a_{2 s} b_{2 s}}+\sum_{k=1}^{s}(-1)^{k} \frac{(4 s+1-2 k) ! !}{(4 s+1) ! !} \delta_{k} \Pi_{2 s-2 k}
$$

\footnotetext{
${ }^{24}$ We are focusing on the even number of boxes $(2 s)$ in the Young tableau, and the odd case can be discussed in the same way.
} 
where we defined a shorthand notation

$$
\delta_{k} \Pi_{2 s-2 k} \equiv \sum_{i_{1} \ldots i_{2 k}}\left\{\left[\delta_{a_{i_{1}} a_{i_{2}}} \ldots \delta_{a_{i_{2 k-1}} a_{i_{2 k}}} \delta_{b_{i_{1}} b_{i_{2}}} \ldots \delta_{b_{i_{2 k-1}} b_{i_{2 k}}}\right]\left[\frac{g_{a_{1} b_{1}} \ldots g_{a_{2 s} b_{2 s}}}{g_{a_{i_{1}} b_{i_{1}}} \ldots g_{a_{i_{2 k}} b_{i_{2 k}}}}\right]\right\} .
$$

Combinatorial factors in (3.61) are determined by requiring the contraction with respect to any pair of indices $\left(a_{i}, a_{j}\right)$ to vanish. For example, observing that ${ }^{25}$

$$
\delta^{a_{1} a_{2}} g_{a_{1} b_{1}} \ldots g_{a_{2 s} b_{2 s}}=\delta_{b_{1} b_{2}} g_{a_{3} b_{3}} \ldots g_{a_{2 s} b_{2 s}}
$$

and

$$
\delta^{a_{1} a_{2}} \delta_{1} \Pi_{2 s-2}=(5+2(2 s-2)) \delta_{b_{1} b_{2}} g_{a_{3} b_{3}} \ldots g_{a_{2 s} b_{2 s}}+(\text { terms with fewer } g),
$$

we conclude that the coefficient in front of the $k=1$ term in (3.61) is indeed $-\frac{1}{4 s+1}$. The other coefficients are determined using induction.

To apply equation (3.61) to the state (3.59), we observe that if all indices $a_{i}=b_{j}=$ 5 , then

$$
\delta_{k} \Pi_{2 s-2 k}=\frac{(2 s) !}{(2 s-2 k) ! s !}\left(g_{55}\right)^{2 s-2 k} .
$$

Substitution into (3.61) gives

$$
\Phi=\left(g_{55}\right)^{2 s}+\sum_{k=1}^{s}(-1)^{k} \frac{(4 s+1-2 k) ! !}{(4 s+1) ! !} \frac{(2 s) !}{(2 s-2 k) ! s !}\left(g_{55}\right)^{2 s-2 k} \propto F\left[-s, \frac{3}{2}+s ; \frac{1}{2} ;\left(g_{55}\right)^{2}\right] .
$$

To relate this answer to the solution (3.9) we observe that equation (3.2) gives

$$
g_{55}=\frac{1-R^{2}}{1+R^{2}}
$$

and that function (3.9) can be rewritten as

$$
\Phi=c_{1} F\left[-\frac{k}{2}, \frac{3+k}{2} ; \frac{1}{2} ;\left(g_{55}\right)^{2}\right]+c_{2} g_{55} F\left[-\frac{k-1}{2}, \frac{4+k}{2} ; \frac{3}{2} ;\left(g_{55}\right)^{2}\right] .
$$

The numerical coefficients $\left(c_{1}, c_{2}\right)$ are such that $c_{1}=0$ for the odd values of $k$, and $c_{2}=0$ for the even ones. Clearly, there is a perfect agreement between (3.64) and (3.65) for $k=2 s$, and the case of odd $k$ can be analyzed in the same way. ${ }^{26}$ Therefore, the algebraic construction (3.10) reproduces the family (3.9). We went though this derivation to illustrate the procedure for analyzing (3.61) in a simple setting, and now we will present the results for more complicated cases. The derivation follows the same logic, but the technical details are more involved, and they are presented in the appendix C.2.

Let us go back to the states in the symmetric representation (3.61), take all indices $b_{k}$ to be equal to five, and allow indices $a_{k}$ to take values from one to four. In other words, we

\footnotetext{
${ }^{25}$ Recall that since $g$ is an element of $\mathrm{SO}(5)$, it satisfies the orthogonality relation $g g^{T}=1$.

${ }^{26}$ One would have to start with a counterpart of equation (3.61) for the representations with an odd number of boxes in the Young tableaux.
} 
are looking at states (3.60). The experience gained in the previous subsections, suggests that it is convenient to use complex coordinates $z_{1}=X_{1}+i X_{2}$ and $z_{2}=X_{3}+i X_{4}$ instead of $X_{j}$, and to accommodate this change of coordinates we define ${ }^{27}$

$$
g_{z 5}=g_{15}+i g_{25}, \quad g_{w 5}=g_{35}+i g_{45} .
$$

To perform contractions of these ingredients one can use the relevant Kronecker symbols

$$
\delta_{z z}=\delta_{x_{1}+i x_{2}, x_{1}+i x_{2}}=0, \quad \delta_{z \bar{z}}=\delta_{x_{1}+i x_{2}, x_{1}-i x_{2}}=2, \quad \delta_{w w}=0, \quad \delta_{w \bar{w}}=2 .
$$

For example, let us consider a wavefunction built only from $g_{z 5}$ and $g_{\bar{z} 5}$. Let us assign charge one to $g_{z 5}$ and charge minus one $g_{\bar{z} 5}$. It is clear that a contraction cannot change the charge of the product, so all terms in (3.61) have the same. Assuming that this charge $q$ is non-negative, we conclude that the last term in (3.61) is equal to $\left(g_{z 5}\right)^{q}$ multiplied by a constant. For other terms one finds a counterpart of (3.63):

$$
\delta_{k} \Pi_{q+2 s-2 k}=2^{k} k !\left[\frac{s !}{k !(s-k) !}\right]\left[\frac{(s+q) !}{k !(s+q-k) !}\right]\left(g_{z 5}\right)^{q+s-k}\left(g_{\bar{z} 5}\right)^{s-k} .
$$

Then the sum (3.61) can be easily performed, and it gives

$$
\Phi=\left(g_{z 5}\right)^{q} F\left[-k, \frac{3}{2}+k+q ; 1+q ;\left(g_{z 5} g_{\bar{z} 5}\right)\right] .
$$

The solution with a negative $q$ is obtained by a making a replacement $g_{z 5} \leftrightarrow g_{\bar{z} 5}$.

Once all $g_{a 5}$ are included, the combinatorics becomes more complicated, but the final result is rather compact:

$$
\begin{aligned}
& \Phi=\left(g_{z 5}\right)^{q_{1}}\left(g_{w 5}\right)^{q_{2}} F_{2}\left[\frac{3}{2}+q_{1}+q_{2}+k_{1}+k_{2},-k_{1},-k_{2} ; q_{1}+1, q_{2}+1 ; g_{z 5} g_{\bar{z} 5}, g_{w 5} g_{\bar{w} 5}\right], \\
& \Lambda=\left(2 k_{1}+2 k_{2}+q_{1}+q_{2}\right)\left(3+2 k_{1}+2 k_{2}+q_{1}+q_{2}\right) .
\end{aligned}
$$

Here $F_{2}$ is the Appell's generalization of the hypergeometric function defined by the series expansion $^{28}$

$$
F_{2}\left[a, b_{1}, b_{2} ; c_{1}, c_{2} ; x, y\right]=\sum_{m, n=0}^{\infty} \frac{(a)_{m+n}\left(b_{1}\right)_{m}\left(b_{2}\right)_{n}}{m ! n !\left(c_{1}\right)_{m}\left(c_{2}\right)_{n}} x^{m} y^{n}
$$

Regularity requires $\left(k_{1}, k_{2}\right)$ to be non-negative integers. Combinatorial derivation of the expression (3.70) is presented in the appendix C.2.

Expression (3.70) reduces to the standard hypergeometric function in the special case (3.69) and its simple extension

$$
\Phi=\left(g_{z 5}\right)^{q_{1}}\left(g_{w 5}\right)^{q_{2}} F\left[-k, \frac{3}{2}+k+q_{1}+q_{2} ; 1+q_{1} ; g_{z 5} g_{\bar{z} 5}\right], \quad s=q_{1}+q_{2}+2 k,
$$

\footnotetext{
${ }^{27}$ We use $(z, w)$ instead of $\left(z_{1}, z_{2}\right)$ to avoid double superscript in various expressions written below. Also, our results will be applicable to solutions which depend on different $(z, w)$ complex structures, for example for $z=X_{1}-i X_{3}, w=X_{2}-i X_{4}$, however, such combinations will not carry specific charges under $[\mathrm{U}(1)]^{4}$ symmetries corresponding to translations in $\left(\alpha_{L}, \beta_{L}, \alpha_{R}, \beta_{R}\right)$.

${ }^{28}$ Recall the standard notation $(a)_{n}=a \ldots(a+n-1)$.
} 
with various permutations of indices $(z, \bar{z}, w, \bar{w})$. Note that solutions (3.70) and (3.72) carry specific $[\mathrm{U}(1)]^{4}$ charges, but their $R$-dependence is rather complicated. This can be seen from the explicit form of the matrix elements:

$$
g_{z 5}=-\frac{2 e^{-2 i \alpha_{L}} z}{1+R^{2}}, \quad g_{w 5}=-\frac{2 e^{-2 i \beta_{L}} w}{1+R^{2}} .
$$

In particular, the solutions described in this subsection don't have the form (3.23), so they cannot be dressed with additional functions of $R$ using the procedure described in section 3.2. Furthermore, all arguments of this subsection are equally applicable to $g_{5 a}$, so by making replacements

$$
g_{a 5} \rightarrow g_{5 a}
$$

in the expressions (3.70), (3.72), one still gets solutions of the Helmholtz equation (3.6) with the same eigenvalues. The resulting separation is perhaps even more impressive since the relevant matrix elements are more complicated

$$
g_{z 5}=\frac{2 e^{-2 i \alpha_{R}}\left(Z_{1-}-y_{+} Z_{2-}\right)}{D}, \quad g_{w 5}=\frac{2 e^{-2 i \beta_{R}}\left(Z_{2-}+y_{+} Z_{1-}\right)}{D} .
$$

We used the convenient notation introduced in (3.13)-(3.14).

We conclude this subsection by counting parameters. There are 12 branches of (3.70) corresponding to different choices of complex structures ${ }^{29}$ and 12 more branches of the solutions flipped by (3.74). Each branch has four integer parameters $\left(q_{1}, q_{2}, k_{1}, k_{2}\right)$. Interestingly, each of the solutions (3.40) dressed with functions of $R$ had four parameters as well. It would be very interesting to find separable solutions with a larger number of free parameters since a general solution in 10 dimensions should be parameterized by ten numbers.

\subsection{Separation in terms of spherical harmonics}

To conclude the discussion of separable solutions in the $\mathrm{SO}(5)$ WZW model, we also mention an alternative parameterization of the group element (3.2) that leads to another set of eigenfunctions depending on four coordinates. This alternative parameterization has a major disadvantage in comparison to (3.2): the Cartan group of $\mathrm{SO}(5) \times \mathrm{SO}(5)$ acts in a complicated way, ${ }^{30}$ so we are discussing it only for completeness.

Let is consider a scalar field on the background of an $\mathrm{SO}(N)$ WZW model. A general group element $g$ can be written as

$$
g=q h,
$$

where $q$ is an element of the subgroup $\mathrm{SO}(N-1)$ and $h$ is a coset representative of $\mathrm{SO}(N) / \mathrm{SO}(N-1)$ which describes the symmetric space $S^{N-1}$. Then the left-invariant one-form frames are given by

$$
\mathcal{L}=g^{-1} d g=h^{-1}\left(d h h^{-1}+q^{-1} d q\right) h \equiv h^{-1} \tilde{\mathcal{L}} h .
$$

\footnotetext{
${ }^{29}$ Here we focus only on the complex structures in $\left(X_{1}, X_{2}, X_{2}, X_{4}\right)$ space. There are also wavefunctions obtained from (3.66) and (3.70) by replacements like $X_{1} \leftrightarrow X_{5}$, but they have more complicated coordinate dependence, so we have not studied them in detail.

${ }^{30}$ Recall that the WZW model on a group $G$ has $G \times G$ global symmetries acting by $g \rightarrow h_{L} g h_{R}$.
} 
The action $(2.1)$, can be rewritten in terms of $\tilde{\mathcal{L}}$,

$$
S=-\frac{k}{2 \pi} \int d^{2} \sigma \eta^{\alpha \beta} \operatorname{tr}\left(\tilde{\mathcal{L}}_{\alpha} \tilde{\mathcal{L}}_{\beta}\right)+\frac{i k}{6 \pi} \int \operatorname{tr}(\tilde{\mathcal{L}} \wedge \tilde{\mathcal{L}} \wedge \tilde{\mathcal{L}}),
$$

so these objects can be used as frames. Introducing a split between the group and the coset,

$$
\tilde{\mathcal{L}}=d h h^{-1}+q^{-1} d q \equiv R_{h}+L_{q}
$$

we can write the frames as a lower triangular block matrix:

$$
\tilde{\mathcal{L}}^{\mathrm{A}}{ }_{M}=\left[\begin{array}{cc}
R^{\alpha} & 0 \\
R^{a}{ }_{\mu} & L^{a}{ }_{m}
\end{array}\right], \quad A=(\alpha, a), \quad M=(\mu, m) .
$$

where we have used the Greek (Latin) indexes are denoting the coset (subgroup) projections. The inverse of this lower triangular block matrix leads to the frames with contravariant indices ${ }^{31}$

$$
\hat{e}_{\mathbf{A}}^{M}=\left[\begin{array}{cc}
R_{\alpha}^{\mu} & 0 \\
-L_{a}^{m} R_{\nu}^{a} R_{\alpha}^{\nu} & L_{a}^{m}
\end{array}\right] \equiv\left[\begin{array}{cc}
R_{\alpha}^{\mu} & 0 \\
N_{\alpha}^{m} & L_{a}^{m}
\end{array}\right]
$$

and the resulting inverse metric has a fibered structure:

$$
G^{M N}=\hat{e}_{\mathbf{A}}^{M} \hat{e}_{\mathbf{B}}^{N} \eta^{\mathbf{A B}}=\eta^{\alpha \beta}\left(R_{\alpha}^{\mu}+N_{\alpha}^{m}\right)\left(R_{\beta}^{\nu}+N_{\beta}^{m}\right)+\eta^{a b} L_{a}^{m} L_{b}{ }^{n} .
$$

In particular, if we look at the Helmholtz equation (3.6) and assume that the scalar $\Phi$ depends only on the coset coordinates, then the problem reduces to the eigenvalue equation on the sphere $S^{N-1}$. The eigenvalues are given by

$$
\Lambda=k(k+N-2),
$$

and eigenfunctions are fully separable in several coordinate systems. For example, writing the metric of $S^{p}$ as

$$
d s_{p}^{2}=d \theta_{p}^{2}+\sin ^{2} \theta_{p} d s_{p-1}^{2},
$$

we can build the eigenfunctions using induction:

$$
\Phi=\prod_{p}^{N-1} \Phi_{p}\left(\theta_{p}\right)
$$

Function $\Phi_{p}\left(\theta_{p}\right)$ satisfies an ordinary differential equation

$$
\frac{1}{\left(\sin \theta_{p}\right)^{p-1}} \frac{d}{d \theta_{p}}\left[\left(\sin \theta_{p}\right)^{p-1} \frac{d \Phi_{p}}{d \theta_{p}}\right]-\frac{\Lambda_{p-1} \Phi_{p}}{\sin ^{2} \theta_{p}}+\Lambda_{p} \Phi_{p}=0 .
$$

The normalizable solutions of this equation can be written in terms of the associated Legendre polynomials:

$$
\Phi_{p}=\left(\sin \theta_{p}\right)^{\frac{2-p}{2}} P_{\lambda}^{(\mu)}\left(\cos \theta_{p}\right), \quad \lambda=k_{p}-1+\frac{p}{2}, \quad \mu=k_{p-1}-1+\frac{p}{2} .
$$

\footnotetext{
${ }^{31}$ Note that $R^{a}{ }_{\nu} R_{\alpha}{ }^{\nu} \neq \delta_{\alpha}^{a}$.
} 
In the case of the $S^{N-1}$ dimensional sphere, the solution depends on $(N-1)$ integer parameters $\left(k_{1}, \ldots k_{N_{1}}\right)$. Interestingly, for $\mathrm{SO}(5)$ we find four parameters, the same number that has been encountered elsewhere in this section, although the spherical separation is very different form the other constructions discussed here. In the $\mathrm{SO}(4)$ case spherical separation gives only three parameters, in contrast to the six-parameter separation encountered in section 2.1. It would be interesting to see whether for larger groups spherical separation becomes more or less powerful than the one coming from parameterizations like (3.1).

\section{Discussion}

In this article we have studied equations for scalar and vector fields on backgrounds of several (gauged) WZW models. While the scalar spectrum has been known for some time $[2,3]$, the algebraic construction of the relevant eigenfunctions turns out to be rather involved [5], and it is desirable to look for a more explicit form of the solutions. Furthermore, the CFT construction of eigenvalues and eigenfunctions [2, 3, 5] does not seem to be easily extendable to vector and tensor fields. To cure these problems, we focused on extracting the wavefunctions directly from the perspective of field equations instead of appealing to algebraic methods.

For the $\mathrm{SO}(4)$ group and its cosets, we demonstrated the full separation of variables in equations for the scalar and vector fields, including the vectors with field strength twisted by the $B$-field. Although we derived separation of variables from the first principles, therefore establishing uniqueness of the separable ansatz for the vector components, we found that the final expressions have the same structure as their counterparts for the rotating black holes [31]. This suggests universality of the form (2.74) for the separable components of vector fields on all geometries that admit separation, and it would be very interesting to test this hypothesis on other backgrounds.

For the $\mathrm{SO}(5)$ group, the full separation of scalar and vector equations seems unlikely, but we found several classes of separable solutions. All our families are parameterized by four integers, in contrast to ten quantum numbers expected for the ten-dimensional geometry. It would be interesting to either find separable families with more parameters or to understand why this can't be done. It would also be interesting to study scalar and vector fields on manifolds corresponding to larger groups and cosets.

\section{Acknowledgments}

This work was supported in part by the DOE grant DE-SC0017962 (OL) and by the UCAS program of the Special Research Associate, as well as the internal funds of the KITS (JT).

\section{A Vector field on the SU(2) WZW model}

In section 2.2.2 we outlined the procedure for finding vectors modes on $\mathrm{SU}(2)$, and in this appendix we present the technical details of this derivation. We will perform the analysis in two steps. In section A.1 we will focus on the standard equations for the vector $((2.42)$ with 
$\zeta=0$ ) and give technical details supporting the steps (i)-(iv) presented in section 2.2.2. This justifies the ansatz (2.57) and prove its uniqueness. In section A.1, we will impose the ansatz (2.57) for the modified vector equation (2.42) and derive the relations (2.67) and (2.68). This would justify step (v) in section 2.2.2.

\section{A.1 Standard equation for the vector: $\zeta=0$}

In this subsection we will focus on $\zeta=0$ case to justify the ansatz (2.57) and prove its uniqueness. To get some intuition about the structure of relevant components we will begin with a special case $n_{1}=n_{2}=\zeta=0$ of the system (2.42), (2.44). Then we extend the analysis to arbitrary values of $\left(n_{1}, n_{2}\right)$.

We begin with recalling that the most general separable solution for a vector field in the geometry (2.43) is given by

$$
C_{i} d x_{i}=e^{i n_{1} \gamma_{L}+i n_{2} \gamma_{R}}\left[V_{y} d y_{1}+V_{1} d \gamma_{L}+V_{2} d \gamma_{R}\right],
$$

where $\left(V_{y}, V_{1}, V_{2}\right)$ are functions of $y_{1}$, which are mixed in equations (2.42). We are looking for combinations of these components that satisfy decoupled equations, and to get insights into the structure of such combinations, we begin with studying a special case:

$$
n_{1}=n_{2}=0, \quad \zeta=0 .
$$

Once these conditions are imposed, the mode $V_{y}$ decouples, it gives $\lambda_{\text {vector }}=0$, while function $V_{y}$ remains arbitrary. This "pure gauge" describes the scalar mode (2.35) in the special case (A.2). To decouple the remaining components, we introduce linear combinations $V_{ \pm}$:

$$
V_{ \pm}=\frac{V_{1} \pm V_{2}}{2}: \quad C_{i} d x_{i}=\left[V_{y} d y_{1}+V_{+}\left(d \gamma_{L}+d \gamma_{R}\right)+V_{-}\left(d \gamma_{L}-d \gamma_{R}\right)\right]
$$

Then the system $(2.42)$ with $\lambda_{\text {vector }} \neq 0$ reduces to differential equations for $V_{ \pm}$

$$
\left(y_{1} \pm 1\right) \frac{d}{d y_{1}}\left[\left(y_{1} \mp 1\right) V_{ \pm}^{\prime}\right]+\lambda_{\text {vector }} V_{ \pm}=0 .
$$

The normalizable solutions are

$$
V_{ \pm}\left(y_{1}\right)=C_{ \pm} F\left[-M, M ; 1 ; \frac{1 \mp y_{1}}{2}\right], \quad \lambda_{\text {vector }}=-M^{2},
$$

where $M$ is a non-negative integer. Comparing this with (2.16) for $n_{+}=n_{-}=0$, we can identify $M$ with $L$ and express the vector modes in terms of the solutions of the scalar equation (2.14):

$$
V_{ \pm}\left(y_{1}\right)=C_{ \pm}\left[\left(1-y_{1}^{2}\right) B_{1}^{\prime}+M\left(y_{1} \pm 1\right) B_{1}\right] .
$$

Then equations (A.4) with $\lambda_{\text {vector }}=-M^{2}$ reduce to an ODE for $B_{1}$ :

$$
\frac{d}{d y_{1}}\left[\left(y_{1}^{2}-1\right) B_{1}^{\prime}\right]-M(M+1) B_{1}=0,
$$

which is a special case of (2.14). 
Next we relax the values $\left(n_{1}, n_{2}\right)$ while keeping $\zeta=0$. In addition to the pure gauge, $C_{i} d x^{i}=d C(x)$, which describes the scalar mode (2.35), we encounter two vector modes, and inspired by (A.3), we write the gauge field as

$$
C_{i} d x_{i}=e^{i n_{1} \gamma_{L}+i n_{2} \gamma_{R}}\left[V_{y} d y_{1}+V_{+}\left(d \gamma_{L}+d \gamma_{R}\right)+V_{-}\left(d \gamma_{L}-d \gamma_{R}\right)\right]
$$

Assuming that $\lambda_{\text {vector }} \neq 0$, we find the expression for $V_{y}$ in terms of $\left(V_{+}, V_{-}\right)$:

$$
V_{y}=-i \frac{\left(n_{1}+n_{2}\right)\left(1-y_{1}\right) V_{+}^{\prime}+\left(n_{1}-n_{2}\right)\left(1+y_{1}\right) V_{-}^{\prime}}{n_{1}^{2}+n_{2}^{2}+\lambda\left(y_{1}^{2}-1\right)-2 n_{1} n_{2} y_{1}} .
$$

It turns out that the components $\left(V_{+}, V_{-}\right)$decouple only if $n_{2}= \pm n_{1}$, so in the general case we write

$$
V_{+}=\hat{V}_{+}+u_{1}\left(n_{1}^{2}-n_{2}^{2}\right) \hat{V}_{-}, \quad V_{-}=\hat{V}_{-}+u_{2}\left(n_{1}^{2}-n_{2}^{2}\right) \hat{V}_{+}
$$

with undetermined constants $\left(u_{1}, u_{2}\right)$. Direct calculations show that equations for $\left(\hat{V}_{+}, \hat{V}_{-}\right)$ decouple only for the specific values of $u_{1,2}$ :

$$
u_{1}=u_{2}=-\frac{1}{\left[\sqrt{\lambda-n_{1}^{2}}+\sqrt{\lambda-n_{2}^{2}}\right]^{2}},
$$

and the eigenvalue problems are

$$
\begin{aligned}
& \frac{d}{d y_{1}}\left[\frac{\left(1-y_{1}^{2}\right) \hat{V}_{-}^{\prime}}{\lambda y_{1}-n_{1} n_{2}-\mu}\right]+\frac{\lambda y_{1}-n_{1} n_{2}+\mu}{\lambda\left(y_{1}^{2}-1\right)} \hat{V}_{-}=0 \\
& \frac{d}{d y_{1}}\left[\frac{\left(1-y_{1}^{2}\right) \hat{V}_{+}^{\prime}}{\lambda y_{1}-n_{1} n_{2}+\mu}\right]+\frac{\lambda y_{1}-n_{1} n_{2}-\mu}{\lambda\left(y_{1}^{2}-1\right)} \hat{V}_{+}=0 .
\end{aligned}
$$

Here we defined

$$
\mu=\sqrt{\left(\lambda-n_{1}^{2}\right)\left(\lambda-n_{2}^{2}\right)} .
$$

Note that the ansatz (A.8) can be rewritten as

$$
C_{i} d x_{i}=e^{i n_{1} \gamma_{L}+i n_{2} \gamma_{R}}\left[V_{y} d y_{1}+q_{+}\left(\hat{V}_{+}+\hat{V}_{-}\right) d \gamma_{L}+q_{-}\left(\hat{V}_{+}-\hat{V}_{-}\right) d \gamma_{R}\right]
$$

where

$$
q_{+}=\left(1+u_{2} n_{1}^{2}-u_{2} n_{2}^{2}\right), \quad q_{-}=\left(1-u_{2} n_{1}^{2}+u_{2} n_{2}^{2}\right)
$$

In particular,

$$
\frac{q_{-}}{q_{+}}=\left[\frac{\lambda-n_{2}^{2}}{\lambda-n_{1}^{2}}\right]^{1 / 2} .
$$

Similar to (A.6), functions $\left(\hat{V}_{+}, \hat{V}_{-}\right)$can be written as

$$
\hat{V}_{ \pm}\left(y_{1}\right)=C_{ \pm}\left[\left(1-y_{1}^{2}\right) B_{1}^{\prime}+\frac{1}{M}\left[\lambda y_{1}-n_{1} n_{2} \pm \mu\right] B_{1}\right],
$$

where function $B_{1}$ satisfied the differential equation (2.14), and parameters $\left(\lambda, \nu_{1}, M\right)$ are related by

$$
\lambda=M^{2}, \quad \nu_{1}=M(M+1) .
$$


The two free parameters $C_{ \pm}$characterize two different degrees of freedom of the vector fields. As demonstrated in section 2.2.2, the expressions for the vector field become especially simple if one considers $C_{+}=C_{-}$or $C_{+}=-C_{-}$(see (2.57)-(2.58) and (2.59)-(2.60)). In the next subsection we will extend these two polarizations to the modified equations for the vector field (2.42) with a nontrivial value of $\zeta$.

\section{A.2 Modified vector equation: arbitrary $\zeta$}

To demonstrate separation of variables and decoupling of various components in equations (2.42) for all values of $\zeta$, we introduce the frames (2.56),

$$
\begin{aligned}
e_{3}^{\mu} \partial_{\mu} & =\partial_{\gamma_{R}}, \quad e_{ \pm}^{\mu} \partial_{\mu}=-\frac{e^{\mp i \gamma_{R}}}{2 \sqrt{1-y_{1}^{2}}}\left[\left(1-y_{1}^{2}\right) \partial_{y_{1}} \pm i\left(y_{1} \partial_{\gamma_{R}}-\partial_{\gamma_{L}}\right)\right] \\
e_{3}^{\mu} e_{3}^{\mu}+\frac{1}{2}\left(e_{+}^{\mu} e_{-}^{\nu}+e_{+}^{\nu} e_{-}^{\mu}\right) & =2 g^{\mu \nu} .
\end{aligned}
$$

and impose the ansatz $(2.57)^{32}$

$$
e_{\mathbf{3}}^{\mu} C_{\mu}=a_{3} e_{\mathbf{3}}^{\mu} \partial_{\mu} Z, \quad e_{+}^{\mu} C_{\mu}=a_{+} e_{+}^{\mu} \partial_{\mu} Z, \quad e_{-}^{\mu} C_{\mu}=a_{-} e_{-}^{\mu} \partial_{\mu} Z .
$$

Here $\left(a_{3}, a_{+}, a_{-}\right)$are undetermined constant coefficients, and function $Z$ is related to the solution of the scalar equation (2.14) by

$$
Z=B_{1} e^{i n_{1} \gamma_{L}+i n_{2} \gamma_{R}}
$$

Such $Z$ after normalization can be expressed in terms of the Wigner's coefficients,

$$
Z=D_{p, q}^{J}, \quad p=n_{2}, \quad q=n_{1},
$$

which obey an important set of identities: ${ }^{33}$

$$
e_{\mathbf{3}}^{\mu} \partial_{\mu} D_{p, q}^{J}=i p D_{p, q}^{J}, \quad e_{+}^{\mu} \partial_{\mu} D_{p, q}^{J}=\frac{1}{2} c_{p} D_{p-1, q}^{J}, \quad e_{-}^{\mu} \partial_{\mu} D_{p, q}^{J}=-\frac{1}{2} c_{p+1} D_{p+1, q}^{J} .
$$

Here we defined

$$
c_{p}=\sqrt{(J+p)(J-p+1)} .
$$

This leads to an alternative form of the ansatz (A.19):

$$
e_{3}^{\mu} C_{\mu}=a_{3} i p D_{p, q}^{J}, \quad e_{+}^{\mu} C_{\mu}=a_{+} \frac{c_{p}}{2} D_{p-1, q}^{J}, \quad e_{-}^{\mu} C_{\mu}=-a_{-} \frac{c_{p+1}}{2} D_{p+1, q}^{J} .
$$

To evaluate the field strength, we need the expressions for the spin connections in the frames (A.19). Although the spin coefficients $\Gamma_{b c}^{a}$,

$$
d e^{a}+\Gamma_{c b}^{a} e^{c} \wedge e^{b}=0
$$

\footnotetext{
${ }^{32} \mathrm{~A}$ similar ansatz for a vector field in a five dimensional black hole with two equal angular momenta was also considered in [62].

${ }^{33}$ Winger's functions are naturally defined with Euler's angle parametrization and one can check these identities explicitly in that parametrization of the group element.
} 
can be easily evaluated from the definition above, it is instructive to compute these coefficients using the group-theoretic analysis. Specifically, substituting the expression for the group element (2.3) into the definition of the left-invariant forms,

$$
\sigma^{a}=\operatorname{Tr}\left(T^{a} g^{-1} d g\right) .
$$

we arrive at a very simple relation

$$
e^{a}=-i \sigma^{a} .
$$

Then the Maurer-Cartan equations for the left-invariant forms lead to the expressions for the spin coefficients in terms of the structure constants of $\mathrm{SU}(2)$ :

$$
d \sigma^{a}+\frac{i}{2} f_{b c}^{a} \sigma^{b} \wedge \sigma^{c}=0 \Rightarrow \Gamma_{b c}^{a}=-\frac{1}{2} f_{b c}^{a} .
$$

These coefficients lead to the expression for the frame components of the modified field strength,

$$
\mathcal{F}_{a b} \equiv F_{a b}+\zeta H_{a b}^{c} C_{c}=\partial_{a} C_{b}-\partial_{b} C_{a}+\left(f_{a b}^{c}+\zeta H_{a b}^{c}\right) C_{c}, \quad H_{+-}^{3}=\frac{i}{4},
$$

and substitution of the ansatz (A.24) and the derivatives (A.22) gives

$$
\begin{aligned}
\mathcal{F}_{+-} & =\frac{1}{4}\left(-c_{p+1}^{2} a_{-}+c_{p}^{2} a_{+}-2 p(1+\zeta) a_{3}\right) D_{p, q}^{J}, \\
\mathcal{F}_{+3} & =\frac{i}{2} c_{p} p\left(a_{3}-(1+\zeta) a_{+}\right) D_{p-1, q}^{J}, \\
\mathcal{F}_{-3} & =\frac{i}{2} c_{p+1} p\left((1-\zeta) a_{-}-a_{3}\right) D_{p+1, q}^{J} .
\end{aligned}
$$

To proceed, we project the equations (2.42) to the frames (A.19). We begin with the derivative of the modified field strength: ${ }^{34}$

$$
\begin{aligned}
\nabla_{\mu} \mathcal{F}^{\alpha \beta} & =\partial_{\mu} \mathcal{F}^{\alpha \beta}+\Gamma_{\mu \lambda}^{\alpha} \mathcal{F}^{\lambda \beta}+\Gamma_{\mu \beta}^{\lambda} \mathcal{F}_{\alpha \lambda} \\
& =\partial_{\mu}\left(\mathcal{F}^{b c} e_{b}^{\alpha} e_{c}^{\beta}\right)+\Gamma_{\mu \lambda}^{\alpha} \mathcal{F}^{b c} e_{b}^{\lambda} e_{c}^{\beta}+\Gamma_{\mu \beta}^{\lambda} \mathcal{F}_{b c} e_{b}^{\alpha} e_{c}^{\lambda} \\
& =\partial_{\mu} \mathcal{F}^{b c} e_{b}^{\alpha} e_{c}^{\beta}+\mathcal{F}^{b c}\left(\nabla_{\mu} e_{b}^{\alpha} e_{c}^{\beta}+\nabla_{\mu} e_{c}^{\beta} e_{b}^{\alpha}\right) .
\end{aligned}
$$

Here we used the relation

$$
\Gamma_{b c}^{a}=e_{\alpha}^{a} e_{b}^{\beta} \nabla_{\beta} e_{c}^{\alpha} .
$$

Substitution of (A.33) into the eigenvalue problem (2.42) gives $^{35}$

$$
\nabla_{\mu} \mathcal{F}^{\mu \beta}+2 \Lambda C^{\beta}=\partial_{a} \mathcal{F}^{a c} e_{c}^{\beta}+\mathcal{F}^{b c}\left(\nabla_{\alpha} e_{b}^{\alpha} e_{c}^{\beta}+e_{b}^{\alpha} \nabla_{\alpha} e_{c}^{\beta}\right)+2 \Lambda C^{\beta}=0 .
$$

Multiplication by $e_{d}^{\beta}$ and summation over $\beta$ gives the final form of the system (2.42) projected to frames: ${ }^{36}$

$$
\partial_{a} \mathcal{F}^{a d}-\frac{1}{2} \mathcal{F}^{b d} f_{a b}^{a}-\frac{1}{2} \mathcal{F}^{b c} f_{b c}^{d}+2 \Lambda C^{d}=0 .
$$

\footnotetext{
${ }^{34}$ In this appendix, we denoting the spacetime indexes with Greek letters, $(\mu, \nu, \ldots)$, and the local frame indexes with Latin letters, $(a, b, \ldots)$.

${ }^{35}$ The factor 2 before $\Lambda$ is for our convenience to compare the result here with the results in the $\left(y, \gamma_{L}, \gamma_{R}\right)$ coordinates.

${ }^{36}$ Recall that $\Gamma_{b c}^{a}=-\frac{1}{2} f a c$.
} 
More explicitly, we find three independent equations:

$$
\begin{aligned}
\partial_{-} \mathcal{F}_{+3}+\partial_{+} \mathcal{F}_{-3}-i \mathcal{F}_{-+}+\frac{\Lambda}{2} C_{3} & =0 \\
\partial_{+} \mathcal{F}_{-+}+\frac{1}{2} \partial_{3} \mathcal{F}_{3+}-\frac{i}{2} \mathcal{F}_{+3}+\frac{\Lambda}{2} C_{+} & =0 \\
\partial_{-} \mathcal{F}_{+-}+\frac{1}{2} \partial_{3} \mathcal{F}_{3-}+\frac{i}{2} \mathcal{F}_{-3}+\frac{\Lambda}{2} C_{-} & =0
\end{aligned}
$$

Substitution of (A.24) and (A.30) reduces (A.37) to a system of algebraic equations for coefficients $\left(a_{3}, a_{+}, a_{-}\right)$:

$$
\begin{aligned}
M\left[\begin{array}{l}
a_{3} \\
a_{+} \\
a_{-}
\end{array}\right] & =\left[\begin{array}{l}
0 \\
0 \\
0
\end{array}\right], \\
M & \equiv\left[\begin{array}{ccc}
p\left[c_{p}^{2}+c_{p+1}^{2}-2(\Lambda-\zeta-1)\right] & -(1+p+\zeta) & 1-p+\zeta \\
2 p(1+p+\zeta) & 2 \Lambda-2 p(p+\zeta)-c_{p}^{2} & c_{p+1}^{2} \\
2 p(1-p+\zeta) & -c_{p}^{2} & 2 p(p-\zeta)-2 \Lambda+c_{p+1}^{2}
\end{array}\right] .
\end{aligned}
$$

Setting the determinant of the matrix $M$ to zero,

$$
[J(J-\zeta)-\Lambda][(1+J)(1+J+\zeta)-\Lambda] \Lambda=0
$$

we find two physical polarizations of the vector field, as well as a pure gauge: ${ }^{37}$

$$
\begin{array}{ll}
\Lambda=J(J-\zeta): & a_{ \pm}=\frac{n_{2}}{n_{2} \pm J} a_{3} ; \\
\Lambda=(1+J)(1+J+\zeta): & a_{ \pm}=\frac{n_{2}}{n_{2} \mp(J+1)} a_{3} ; \\
\Lambda=0: & a_{-}=a_{+}=a_{3} .
\end{array}
$$

The last line gives $F_{\mu \nu}=0$, but $\mathcal{F}_{\mu \nu}$ is still nontrivial:

$$
\mathcal{F}_{+-}=-\frac{p \zeta}{2} a_{3} D_{p, q}^{J}, \quad \mathcal{F}_{+3}=-\frac{i p \zeta}{2} c_{p} a_{3} D_{p-1, q}^{J}, \quad \mathcal{F}_{-3}=-\frac{i p \zeta}{2} c_{p+1} a_{3} D_{p+1, q}^{J} .
$$

This field is divergence-free, so it gives $\Lambda=0$.

To threat the first two solutions from (A.40) in a unified fashion, we observe that $Z=D_{n_{2}, n_{1}}^{J}$ is a solution of the scalar equation (2.14) with an eigenvalue

$$
\nu_{1}=J(J+1), \quad J \geq 0
$$

The last expression can be rewritten as

$$
\nu_{1}=M(M+1) \quad \text { with } \quad M=J \quad \text { or } \quad M=-J-1 .
$$

\footnotetext{
${ }^{37}$ Here we recalled the definition (A.21) to go back from parameter $p$ to $n_{2}$, which is used in the main body of the paper.
} 
This concluded the derivation of the vector eigenvalues (2.68). Taking the first option for the first line in (A.40) and the second option for the second line, we can summarize both branches as

$$
\Lambda=M(M-\zeta): \quad a_{ \pm}=\frac{n_{2}}{n_{2} \pm M} a_{3} \quad \nu_{1}=M(M+1),
$$

where $M$ can take both positive and negative values. This concludes the derivation of the vector eigenvalues (2.68).

Although it is not obvious a priori, it is straightforward to check that the solutions (A.24) (A.44) satisfy the "Lorenz gauge" condition: ${ }^{38}$

$$
\nabla_{\mu} C^{\mu}=\nabla_{a} C^{a}=2 n_{2}^{2} a_{3}+\left(J+1+n_{2}\right)\left(J-n_{2}\right) a_{-}+\left(J+n_{2}\right)\left(J+1-n_{2}\right) a_{+}=0 .
$$

This fact plays an important role in section 2.2, where the $\mathrm{SU}(2)$ vectors are combined to produce separable solutions for the vector fields on $\mathrm{SO}(4)$.

This method can be easily generalized to model with higher rank semi-simple groups. Take a highest weight representation in which states can be labelled by their weight $\vec{r}$. The proper ansatz of the vector field will be

$$
e_{\mu}^{H_{i}} \partial^{\mu} A_{\mu}=\sum_{\vec{r}} a_{H_{i}} D_{\vec{r}}, \quad e_{\mu}^{E_{\vec{\alpha}_{i}}} \partial^{\mu} A_{\mu}=a_{E_{\vec{\alpha}_{i}}} D_{\vec{r}-\vec{\alpha}_{i}},
$$

where $H_{i}$ correspond to the Cartan subgroup (the analogue of $T_{3}$ ), $\vec{\alpha}_{i}$ correspond to the roots (the analogue of $T_{ \pm}$) and $D_{\vec{r}}$ are the higher dimensional analogue of Wigner's functions. Using the general commutation relations between the Cartan and root operators, one can see that indeed that different modes will not mix in any components of the field strength if we assume there are no degeneracies. However to derive the corresponding eigenvalue problem is very cumbersome.

\section{B Geometries for the gauged WZW models}

The gauged WZW on coset $G / H$ can be constructed by integrating out the gauge fields $A_{ \pm}$corresponding to the subgroup $H$ from WZW on $G$. In this appendix, we present the construction of this geometric background. It is convenient to separate the generators $T^{A}$ of the group $G$ into $T^{a}$ corresponding to the subgroup $H$ and $T^{\alpha}$ corresponding to the coset $G / H$ and define the left and right Maurer-Cartan forms on $G$ as

$$
\begin{aligned}
& L_{M}^{A}=-i \operatorname{Tr}\left(T^{A} g^{-1} \partial_{M} g\right), \quad R_{\mu}^{A}=-i \operatorname{Tr}\left(T^{A} \partial_{M} g g^{-1}\right), \\
& R_{M}^{A}=D_{A B} L_{M}^{B}, \quad D_{A B}=\operatorname{Tr}\left(T_{A} g T_{B} g^{-1}\right), \quad g \in G .
\end{aligned}
$$

In terms of the left and right forms the corresponding metric, Kalb-Ramond and dilaton field of the gauged WZW are given by

$$
\begin{aligned}
G_{\mu \nu} & =\frac{k}{2 \pi}\left(\eta_{A B} L_{\mu}^{A} L_{\nu}^{B}-L_{\mu}^{a}\left(D_{a b}-\eta_{a b}\right)^{-1} R_{\nu}^{b}-R_{a}\left(D_{a b}-\eta_{a b}\right)^{T} L_{\nu}^{b}\right), \\
B_{\mu \nu} & =\frac{k}{2 \pi}\left(B_{\mu \nu}^{0}-L_{\mu}^{a}\left(D_{a b}-\eta_{a b}\right)^{-1} R_{\nu}^{b}+R_{a}\left(D_{a b}-\eta_{a b}\right)^{T} L_{\nu}^{b}\right), \\
e^{-2 \Phi} & =\operatorname{det}\left(D_{a b}-\eta_{a b}\right),
\end{aligned}
$$

\footnotetext{
${ }^{38}$ Since we are dealing with a massive vector field $C$, the equations (2.42) are not invariant under the $C \rightarrow C+d f$ transformations.
} 
where $B^{0}$ is defined by $H^{0}=d B^{0}$ with

$$
H_{M N P}^{0}=f_{A B C} L_{M}^{A} L_{N}^{B} L_{P}^{C}, \quad f_{A B C}=-i \operatorname{Tr}\left(\left[T_{A}, T_{B}\right] T_{C}\right) .
$$

After some manipulations, the metric can also be written in terms of local frames as

$$
\begin{aligned}
G_{\mu \nu} & =\frac{k}{2 \pi} \eta_{\alpha \beta} e_{\mu}^{\alpha} e_{\nu}^{\beta}=\frac{k}{2 \pi} \eta_{\alpha \beta} \hat{e}_{\mu}^{\alpha} \hat{e}_{\nu}^{\beta}, \\
e_{\mu}^{\alpha} & =L_{\mu}^{\alpha}-D_{a \alpha}^{T}\left(D_{a b}-\eta_{a b}\right)^{-T} L_{\mu}^{b}, \quad \hat{e}_{\mu}^{\alpha}=R_{\mu}^{\alpha}-D_{\alpha a}\left(D_{a b}-\eta_{a b}\right)^{-1} R_{\mu}^{b} .
\end{aligned}
$$

In the group model (2.1), there are also two natural local frames $L_{M}^{A}$ and $R_{M}^{A}$ which are related to the $G_{L} \times G_{R}$ isometries of the group model. By gauging the subgroup $H$, the $G_{L} \times G_{R}$ isometries are mostly explicitly broken and the resulted gWZW model should only depend on the gauge invariant quantities. As a result the two local frames (B.6) of gWZW are gauge invariant projections and restrictions of $L_{M}^{A}$ and $R_{M}^{A}$. Therefore the two local frames (B.6) are the ones (after some linear combinations) we used in our separable ansatz (2.57) and (2.105) inherited from the general form (A.46).

\section{Scalar wavefunctions for the $\mathrm{SO}(5)$ sigma model}

In this appendix we provide some technical details relevant for deriving several classes of scalar eigenfunctions on the background of the SO(5) WZW model.

\section{C.1 Solutions with linear $z$-dependence}

In section 3.4 we outlined the procedure for starting with a general expression (3.45) for the eigenfunction linear in $\left(X_{1}, X_{2}, X_{2}, X_{4}\right)$ and using various algebraic relations following from the Helmholtz equation (3.6) to derive the options (3.6) that correspond to two types of branches (3.50). In this appendix, we will begin with (3.50) and derive the expressions for functions $\left(g_{1}, g_{2}, g_{3}, g_{4}\right)$. We will focus on the first option in (3.50), and the second one can be analyzed in the same way.

Let us define a convenient function

$$
F=\left(1+R^{2}\right)^{q}\left(\nabla^{2}+\Lambda\right) \Psi .
$$

Once the first solution from (3.50),

$$
n_{2}=n_{1}-1, n_{3}=n_{4}: \Psi=\frac{E_{n_{1}, n_{2}, n_{3}, n_{4}}}{\left(1+R^{2}\right)^{q}}\left[g_{1}\left(Y_{1}, Y_{2}\right) \bar{z}_{1}+g_{2}\left(Y_{1}, Y_{2}\right) z_{2}\right],
$$

is substituted, function $F$ must vanish. This leads to complicated overdetermined equations for $g_{1}$ and $g_{2}$, but one of the necessary conditions is very simple:

$$
\left.\frac{\partial^{2} F}{\partial X_{3}^{2}}\right|_{X_{1}=i X_{2}}=0: \quad\left[\left(1+Y_{2}^{2}\right) \partial_{Y_{2}}+\left(1+Y_{1}^{2}\right) \partial_{Y_{1}}\right] g_{2}=0
$$

The most general solution of the resulting first order PDE is

$$
g_{2}=h_{2}\left[\frac{Y_{1}-Y_{2}}{1+Y_{1} Y_{2}}\right]
$$


where $h_{2}$ is an arbitrary function of its argument. Next we look at another necessary condition:

$$
\left.\frac{\partial}{\partial X_{4}}\left(\left.F\right|_{X_{3}=-i X_{4}}\right)\right|_{X_{4}=0}=0: \quad\left[\left(1+Y_{2}^{2}\right) \partial_{Y_{2}}+\left(1+Y_{1}^{2}\right) \partial_{Y_{1}}\right] g_{1}=0 .
$$

This gives

$$
g_{1}=h_{1}\left[\frac{Y_{1}-Y_{2}}{1+Y_{1} Y_{2}}\right] .
$$

All remaining equations contain $\left(Y_{1}, Y_{2}\right)$ only in one combination

$$
y_{-}=\frac{Y_{1}-Y_{2}}{1+Y_{1} Y_{2}}
$$

so we find an overdetermined system of ordinary differential equations for $\left(h_{1}\left[y_{-}\right], h_{2}\left[y_{-}\right]\right)$ with coefficients depending on $\left(X_{1}, X_{2}, X_{2}, X_{4}\right)$. One of these equations gives the expression (3.52) for $h_{1}$ in terms of $h_{2}$, then the consistency of the remaining system for $h_{2}$ leads to two options for $\Lambda$ :

$$
\Lambda=2 q(q+1) \quad \text { or } \quad \Lambda=2 q(q+2) .
$$

In both cases, the system collapses to a single hypergeometric equation for $h_{2}$, and the solutions are given by (3.53).

The second option in (3.50) can be analyzed in the same way, and the result is $(3.55)-(3.56)$.

\section{C.2 Wavefunctions in the symmetric representations}

The goal of this subsection is to derive the expression (3.70) from the general result (3.61). To do so, we focus on the wavefunction (3.61) containing four building blocks: $\left(g_{z 5}, g_{\bar{z} 5}, g_{w 5}, g_{\bar{w} 5}\right)$. To evaluate the relevant combinatorial factors, it is convenient to white the leading term in (3.61) more explicitly as

$$
g_{a_{1} 5} \ldots g_{a_{2 s} 5} \rightarrow\left[g_{z 5}\right]^{q_{1}}\left[g_{w 5}\right]^{q_{2}} x^{s_{1}} y^{s_{2}},
$$

where we defined

$$
x=g_{z 5} g_{\bar{z} 5}, \quad y=g_{w 5} g_{\bar{w} 5}
$$

Furthermore, relations (3.67) ensure that the only two types of contractions in (3.61): $g_{z 5}$ with $g_{\bar{z} 5}$ and $g_{w 5}$ with $g_{\bar{w} 5}$. Therefore, the index $k$ in (3.61) can be decomposed as $k=k_{1}+k_{2}$ to keep track of the contractions of the first and the second types. With this notation we find

$$
\begin{aligned}
& \frac{\delta_{k} \Pi_{2 s-2 k}}{\left[g_{z 5}\right]^{q_{1}}\left[g_{w 5}\right]^{q_{2}} x^{s_{1}} y^{s_{2}}}=\sum_{p=0}^{k}\left\{2^{p} p !\left[\frac{\left(s_{1}+q_{1}\right) !}{p !\left(s_{1}+q_{1}-p\right) !}\right]\left[\frac{s_{1} !}{p !\left(s_{1}-p\right) !}\right]\right. \\
& \left.\times 2^{k-p}(k-p) !\left[\frac{\left(s_{2}+q_{2}\right) !}{(k-p) !\left(s_{2}+q_{2}-k+p\right) !}\right]\left[\frac{s_{2} !}{(k-p) !\left(s_{2}-k+p\right) !}\right] \frac{1}{x^{p} y^{k-p}}\right\} \\
& =2^{k} \sum_{p=0}^{k} \frac{x^{-p} y^{p-k}}{p !(k-p) !} \frac{\left(s_{1}+q_{1}\right) !}{\left(s_{1}+q_{1}-p\right) !} \frac{s_{1} !}{\left(s_{1}-p\right) !} \frac{\left(s_{2}+q_{2}\right) !}{\left(s_{2}+q_{2}-k+p\right) !} \frac{s_{2} !}{\left(s_{2}-k+p\right) !}
\end{aligned}
$$


Substitution into (3.61) gives

$$
\begin{aligned}
\Phi= & \sum_{k=0}^{s}(-1)^{k} \frac{(4 s+1-2 k) ! !}{(4 s+1) ! !} \delta_{k} \Pi_{2 s-2 k} \\
= & {\left[g_{z 5}\right]^{q_{1}}\left[g_{w 5}\right]^{q_{2}} \sum_{k=0}^{s}(-1)^{k} \frac{(4 s+1-2 k) ! !}{(4 s+1) ! !} 2^{k} } \\
& \times \sum_{p=0}^{k} \frac{x^{s_{1}-p} y^{s_{2}+p-k}}{p !(k-p) !} \frac{\left(s_{1}+q_{1}\right) !}{\left(s_{1}+q_{1}-p\right) !} \frac{s_{1} !}{\left(s_{1}-p\right) !} \frac{\left(s_{2}+q_{2}\right) !}{\left(s_{2}+q_{2}-k+p\right) !} \frac{s_{2} !}{\left(s_{2}-k+p\right) !} \\
= & {\left[g_{z 5}\right]^{q_{1}}\left[g_{w 5}\right]^{q_{2}} \sum_{m, n} \frac{x^{m} y^{n}}{m ! n !(-2)^{m+n-s_{1}-s_{2}}} \frac{\left(4 s+1+2 m+2 n-2 s_{1}-2 s_{2}\right) ! !}{(4 s+1) ! !} } \\
& \times \frac{\left(-s_{1}\right)_{m}}{(-1)^{m}} \frac{\left(-s_{2}\right)_{n}}{(-1)^{n}} \frac{\left(s_{1}+q_{1}\right) !}{\left(m+q_{1}\right) !} \frac{\left(s_{2}+q_{2}\right) !}{\left(n+q_{2}\right) !}
\end{aligned}
$$

At the last stage we used the relation

$$
\left(-s_{1}\right)_{m}=\left(-s_{1}\right)\left(-s_{1}+1\right) \ldots\left(-s_{1}+m-1\right)=(-1)^{m} \frac{s_{1} !}{\left(s_{1}-m\right) !}
$$

for the coefficients $(a)_{n}=a \ldots(a+n-1)$ which appear in the definition of the hypergeometric function and its generalization (3.71). To simplify the expression (C.12), we observe that the definition of $s$ from (3.61) implies $s=s_{1}+s_{2}+\frac{q_{1}+q_{2}}{2}$. Then

$$
\begin{gathered}
\left(4 s+1+2 m+2 n-2 s_{1}-2 s_{2}\right) ! !=\left(2\left[s_{1}+s_{2}+q_{1}+q_{2}\right]+2[m+n]+1\right) ! ! \\
=2^{m+n}\left(s_{1}+s_{2}+q_{1}+q_{2}+\frac{3}{2}\right)_{m+n}\left(2\left[s_{1}+s_{2}+q_{1}+q_{2}\right]+1\right) ! !
\end{gathered}
$$

Substituting this into (C.12) and dropping a complicated but irrelevant overall factor which depends on $\left(s_{1}, s_{2}, q_{1}, q_{2}\right)$, we find ${ }^{39}$

$$
\begin{aligned}
\Phi & \propto\left[g_{z 5}\right]^{q_{1}}\left[g_{w 5}\right]^{q_{2}} \sum_{m, n} \frac{x^{m} y^{n}}{m ! n !} \frac{\left(s_{1}+s_{2}+q_{1}+q_{2}+\frac{3}{2}\right)_{m+n}}{\left(q_{1}+1\right)_{m}\left(q_{2}+1\right)_{n}}\left(-s_{1}\right)_{m}\left(-s_{2}\right)_{n} \\
& \propto\left[g_{z 5}\right]^{q_{1}}\left[g_{w 5}\right]^{q_{2}} F_{2}\left[\frac{3}{2}+q_{1}+q_{2}+s_{1}+s_{2},-s_{1},-s_{2} ; q_{1}+1, q_{2}+1 ; x, y\right] .
\end{aligned}
$$

Here we used the definition (3.71) of the Appell series. Equation (C.15) completes the derivation of the wavefunction (3.70).

We conclude this subsection by justifying the wavefunction (3.72) for the special situation of $s_{2}=0$. In this case the Appell series reduce to the standard hypergeometric function, but one can also easily derive the result (3.72) from the first principles without appealing to the reduction formulas. For the solution with $s_{2}=0$, the contractions can happen only between $g_{z 5}$ and $g_{\bar{z} 5}$, so the contraction rule becomes

$$
\delta_{p} \Pi_{2 s-2 p}=2^{p} p !\left[\frac{\left(s_{1}+q_{1}\right) !}{p !\left(s_{1}+q_{1}-p\right) !}\right]\left[\frac{s_{1} !}{p !\left(s_{1}-p\right) !}\right] x^{s_{1}-p}\left(g_{z 5}\right)^{q_{1}}\left(g_{w 5}\right)^{q_{2}} .
$$

\footnotetext{
${ }^{39}$ To arrive at this expression we also used the relations $\left(m+q_{1}\right) !=\left(q_{1}+1\right)_{m} q_{1}$ ! and $\left(n+q_{2}\right) !=\left(q_{2}+1\right)_{n} q_{2}$ !
} 
Then the summation (3.61) gives

$$
\begin{aligned}
\Phi & =\left(g_{z 5}\right)^{q_{1}}\left(g_{w 5}\right)^{q_{2}} \sum_{k}(-2)^{k} \frac{(4 s+1-2 k) ! !}{(4 s+1) ! !} \frac{x^{s_{1}-k}}{k !} \frac{\left(s_{1}+q_{1}\right) !}{\left(s_{1}+q_{1}-k\right) !} \frac{s_{1} !}{\left(s_{1}-k\right) !} \\
& \propto\left(g_{z 5}\right)^{q_{1}}\left(g_{w 5}\right)^{q_{2}} \sum_{n} \frac{1}{(-2)^{n}} \frac{\left(2\left[s_{1}+q_{1}+q_{2}\right]+2 n+1\right) ! !}{(4 s+1) ! !} \frac{x^{n}}{\left(s_{1}-n\right) !} \frac{\left(s_{1}+q_{1}\right) !}{\left(n+q_{1}\right) !} \frac{s_{1} !}{n !} \\
& \propto\left(g_{z 5}\right)^{q_{1}}\left(g_{w 5}\right)^{q_{2}} \sum_{n} \frac{\left(s_{1}+q_{1}+q_{2}+\frac{3}{2}\right)_{n}\left(-s_{1}\right)_{n}}{\left(n+q_{1}\right) !} \frac{x^{n}}{n !} .
\end{aligned}
$$

We used the expressions (C.13) and (C.14) for the factorials and double factorials in terms of the gamma functions, and, as before, we dropped the complicated but irrelevant constant prefactors in front of $\Phi$. Performing the sum in the last expression, we arrive at the final result (3.72):

$$
\Phi=\left(g_{w 5}\right)^{q_{2}}\left(g_{z 5}\right)^{q_{1}} F\left[-k, \frac{3}{2}+k+q_{1}+q_{2} ; 1+q_{1} ; g_{z 5} g_{\bar{z} 5}\right], \quad s=q_{1}+q_{2}+2 k .
$$

This concludes our justification of various combinatorial formulas used in section 3.5.

Open Access. This article is distributed under the terms of the Creative Commons Attribution License (CC-BY 4.0), which permits any use, distribution and reproduction in any medium, provided the original author(s) and source are credited.

\section{References}

[1] E. Witten, NonAbelian Bosonization in Two-Dimensions, Commun. Math. Phys. 92 (1984) 455 [INSPIRE].

[2] A.P. Polychronakos and K. Sfetsos, Solving field equations in non-isometric coset CFT backgrounds, Nucl. Phys. B 840 (2010) 534 [arXiv:1006.2386] [InSPIRE].

[3] A.P. Polychronakos and K. Sfetsos, High spin limits and non-Abelian T-duality, Nucl. Phys. B 843 (2011) 344 [arXiv: 1008.3909] [InSPIRE].

[4] R. Dijkgraaf, H.L. Verlinde and E.P. Verlinde, String propagation in a black hole geometry, Nucl. Phys. B 371 (1992) 269 [inSPIRE].

[5] O. Lunin and W. Tian, Scalar fields on $\lambda$-deformed cosets, Nucl. Phys. B 938 (2019) 671 [arXiv: 1808.02971] [INSPIRE].

[6] O. Lunin and J. Tian, Supergravity excitations of the WZW models, to appear.

[7] B. Carter, Global structure of the Kerr family of gravitational fields, Phys. Rev. 174 (1968) 1559 [INSPIRE].

[8] B. Carter, Hamilton-Jacobi and Schrödinger separable solutions of Einstein's equations, Commun. Math. Phys. 10 (1968) 280 [inSPIRE].

[9] M. Walker and R. Penrose, On quadratic first integrals of the geodesic equations for type \{22\} spacetimes, Commun. Math. Phys. 18 (1970) 265 [INSPIRE].

[10] Z.W. Chong, G.W. Gibbons, H. Lü and C.N. Pope, Separability and Killing tensors in Kerr-Taub-NUT-de Sitter metrics in higher dimensions, Phys. Lett. B 609 (2005) 124 [hep-th/0405061] [INSPIRE]. 
[11] M. Vasudevan, K.A. Stevens and D.N. Page, Separability of the Hamilton-Jacobi and Klein-Gordon equations in Kerr-de Sitter metrics, Class. Quant. Grav. 22 (2005) 339 [gr-qc/0405125] [INSPIRE].

[12] W. Chen, H. Lü and C.N. Pope, Separability in cohomogeneity-2 Kerr-NUT-AdS metrics, JHEP 04 (2006) 008 [hep-th/0602084] [INSPIRE].

[13] V.P. Frolov and D. Kubiznak, Hidden Symmetries of Higher Dimensional Rotating Black Holes, Phys. Rev. Lett. 98 (2007) 011101 [gr-qc/0605058] [INSPIRE].

[14] D.N. Page, D. Kubiznak, M. Vasudevan and P. Krtous, Complete integrability of geodesic motion in general Kerr-NUT-AdS spacetimes, Phys. Rev. Lett. 98 (2007) 061102 [hep-th/0611083] [INSPIRE].

[15] V.P. Frolov, P. Krtous and D. Kubiznak, Separability of Hamilton-Jacobi and Klein-Gordon Equations in General Kerr-NUT-AdS Spacetimes, JHEP 02 (2007) 005 [hep-th/0611245] [INSPIRE].

[16] P. Krtous, D. Kubiznak, D.N. Page and V.P. Frolov, Killing-Yano Tensors, Rank-2 Killing Tensors, and Conserved Quantities in Higher Dimensions, JHEP 02 (2007) 004 [hep-th/0612029] [INSPIRE].

[17] V.P. Frolov and D. Kubiznak, Higher-Dimensional Black Holes: Hidden Symmetries and Separation of Variables, Class. Quant. Grav. 25 (2008) 154005 [arXiv:0802.0322] [InSPIRE].

[18] P. Krtous, V.P. Frolov and D. Kubiznak, Hidden Symmetries of Higher Dimensional Black Holes and Uniqueness of the Kerr-NUT-(A)dS spacetime, Phys. Rev. D 78 (2008) 064022 [arXiv: 0804.4705] [INSPIRE].

[19] T. Houri, D. Kubiznak, C.M. Warnick and Y. Yasui, Generalized hidden symmetries and the Kerr-Sen black hole, JHEP 07 (2010) 055 [arXiv: 1004.1032] [INSPIRE].

[20] D. Kubiznak, C.M. Warnick and P. Krtous, Hidden symmetry in the presence of fluxes, Nucl. Phys. B 844 (2011) 185 [arXiv: 1009.2767] [inSPIRE].

[21] M. Cariglia, P. Krtous and D. Kubiznak, Commuting symmetry operators of the Dirac equation, Killing-Yano and Schouten-Nijenhuis brackets, Phys. Rev. D 84 (2011) 024004 [arXiv:1102.4501] [INSPIRE].

[22] M. Cariglia, P. Krtous and D. Kubiznak, Dirac Equation in Kerr-NUT-(A)dS Spacetimes: Intrinsic Characterization of Separability in All Dimensions, Phys. Rev. D 84 (2011) 024008 [arXiv: 1104.4123] [INSPIRE].

[23] D. Kubiznak and M. Cariglia, On Integrability of spinning particle motion in higher-dimensional black hole spacetimes, Phys. Rev. Lett. 108 (2012) 051104 [arXiv:1110.0495] [INSPIRE].

[24] V. Frolov, P. Krtous and D. Kubiznak, Black holes, hidden symmetries, and complete integrability, Living Rev. Rel. 20 (2017) 6 [arXiv:1705.05482] [INSPIRE].

[25] D. Kubiznak, H.K. Kunduri and Y. Yasui, Generalized Killing-Yano equations in D $=5$ gauged supergravity, Phys. Lett. B 678 (2009) 240 [arXiv:0905.0722] [INSPIRE].

[26] T. Houri, D. Kubiznak, C. Warnick and Y. Yasui, Symmetries of the Dirac Operator with Skew-Symmetric Torsion, Class. Quant. Grav. 27 (2010) 185019 [arXiv:1002.3616] [INSPIRE]. 
[27] T. Houri, D. Kubiznak, C.M. Warnick and Y. Yasui, Generalized hidden symmetries and the Kerr-Sen black hole, JHEP 07 (2010) 055 [arXiv: 1004.1032] [INSPIRE].

[28] Y. Chervonyi and O. Lunin, Killing(-Yano) Tensors in String Theory, JHEP 09 (2015) 182 [arXiv: 1505.06154] [INSPIRE].

[29] S.A. Teukolsky, Rotating black holes: separable wave equations for gravitational and electromagnetic perturbations, Phys. Rev. Lett. 29 (1972) 1114 [INSPIRE].

[30] S.A. Teukolsky, Perturbations of a rotating black hole. 1. Fundamental equations for gravitational electromagnetic and neutrino field perturbations, Astrophys. J. 185 (1973) 635 [INSPIRE].

[31] O. Lunin, Maxwell's equations in the Myers-Perry geometry, JHEP 12 (2017) 138 [arXiv: 1708.06766] [INSPIRE].

[32] V.P. Frolov, P. Krtouš and D. Kubizňák, Separation of variables in Maxwell equations in Plebański-Demiański spacetime, Phys. Rev. D 97 (2018) 101701 [arXiv:1802.09491] [INSPIRE].

[33] P. Krtouš, V.P. Frolov and D. Kubizňák, Separation of Maxwell equations in Kerr-NUT-(A)dS spacetimes, Nucl. Phys. B 934 (2018) 7 [arXiv: 1803.02485] [InSPIRE].

[34] R. Cayuso, F. Gray, D. Kubizňák, A. Margalit, R. Gomes Souza and L. Thiele, Principal Tensor Strikes Again: Separability of Vector Equations with Torsion, Phys. Lett. B 795 (2019) 650 [arXiv: 1906.10072] [INSPIRE].

[35] T. Houri, N. Tanahashi and Y. Yasui, On symmetry operators for the Maxwell equation on the Kerr-NUT-(A)dS spacetime, Class. Quant. Grav. 37 (2020) 015011 [arXiv:1908.10250] [INSPIRE].

[36] O. Lunin, Excitations of the Myers-Perry Black Holes, JHEP 10 (2019) 030 [arXiv: 1907.03820] [INSPIRE].

[37] E. Witten, On string theory and black holes, Phys. Rev. D 44 (1991) 314 [inSPIRE].

[38] I. Affleck, Exact Critical Exponents for Quantum Spin Chains, Nonlinear Sigma Models at $\theta=\pi$ and the Quantum Hall Effect, Nucl. Phys. B 265 (1986) 409 [InSPIRE].

[39] K. Sfetsos, Integrable interpolations: From exact CFTs to non-Abelian T-duals, Nucl. Phys. $B \mathbf{8 8 0}(2014) 225$ [arXiv: 1312.4560] [InSPIRE].

[40] K. Sfetsos and D.C. Thompson, Spacetimes for $\lambda$-deformations, JHEP 12 (2014) 164 [arXiv: 1410.1886] [INSPIRE].

[41] S. Demulder, K. Sfetsos and D.C. Thompson, Integrable $\lambda$-deformations: Squashing Coset CFTs and $A d S_{5} \times S^{5}$, JHEP 07 (2015) 019 [arXiv: 1504.02781] [INSPIRE].

[42] K. Sfetsos, K. Siampos and D.C. Thompson, Generalised integrable $\lambda$ - and $\eta$-deformations and their relation, Nucl. Phys. B 899 (2015) 489 [arXiv:1506.05784] [InSPIRE].

[43] T.J. Hollowood, J.L. Miramontes and D.M. Schmidtt, Integrable Deformations of Strings on Symmetric Spaces, JHEP 11 (2014) 009 [arXiv: 1407.2840] [INSPIRE].

[44] T.J. Hollowood, J.L. Miramontes and D.M. Schmidtt, An Integrable Deformation of the $A d S_{5} \times S^{5}$ Superstring, J. Phys. A 47 (2014) 495402 [arXiv:1409.1538] [InSPIRE].

[45] C. Appadu and T.J. Hollowood, $\beta$-function of $k$ deformed $A d S_{5} \times S^{5}$ string theory, JHEP 11 (2015) 095 [arXiv: 1507.05420] [INSPIRE]. 
[46] B. Hoare and A.A. Tseytlin, On integrable deformations of superstring sigma models related to $A d S_{n} \times S^{n}$ supercosets, Nucl. Phys. B 897 (2015) 448 [arXiv: 1504.07213] [InSPIRE].

[47] R. Borsato, A.A. Tseytlin and L. Wulff, Supergravity background of $\lambda$-deformed model for $A d S_{2} \times S^{2}$ supercoset, Nucl. Phys. B 905 (2016) 264 [arXiv:1601.08192] [InSPIRE].

[48] Y. Chervonyi and O. Lunin, Supergravity background of the $\lambda$-deformed $A d S_{3} \times S^{3}$ supercoset, Nucl. Phys. B 910 (2016) 685 [arXiv: 1606.00394] [INSPIRE].

[49] R. Borsato and L. Wulff, Target space supergeometry of $\eta$ and $\lambda$-deformed strings, JHEP 10 (2016) 045 [arXiv: 1608.03570] [inSPIRE].

[50] G. Georgiou, K. Sfetsos and K. Siampos, All-loop anomalous dimensions in integrable $\lambda$-deformed $\sigma$-models, Nucl. Phys. B 901 (2015) 40 [arXiv:1509.02946] [InSPIRE].

[51] Y. Chervonyi and O. Lunin, Generalized $\lambda$-deformations of $A d S_{p} \times S^{p}$, Nucl. Phys. B 913 (2016) 912 [arXiv: 1608.06641] [InSPIRE].

[52] S. Demulder, S. Driezen, A. Sevrin and D.C. Thompson, Classical and Quantum Aspects of Yang-Baxter Wess-Zumino Models, JHEP 03 (2018) 041 [arXiv:1711.00084] [INSPIRE].

[53] O. Lunin and W. Tian, Analytical structure of the generalized $\lambda$-deformation, Nucl. Phys. $B$ 929 (2018) 330 [arXiv: 1711.02735] [INSPIRE].

[54] C. Appadu, T.J. Hollowood, D. Price and D.C. Thompson, Quantum Anisotropic Sigma and Lambda Models as Spin Chains, J. Phys. A 51 (2018) 405401 [arXiv:1802.06016] [INSPIRE].

[55] K. Yano, Some Remarks on Tensor Fields and Curvature, Ann. Math. 55 (1952) 328.

[56] S.-i. Tachibana, On conformal Killing tensor in a Riemannian space, Tohoku Math. J. 21 (1969) 56.

[57] T. Kashiwada, On conformal Killing tensor, Nat. Sci. Rep. Ochanomizu Univ. 19 (1968) 67 [INSPIRE].

[58] R.C. Myers and M.J. Perry, Black Holes in Higher Dimensional Space-Times, Annals Phys. 172 (1986) 304 [INSPIRE].

[59] I. Bars and K. Sfetsos, Generalized duality and singular strings in higher dimensions, Mod. Phys. Lett. A 7 (1992) 1091 [hep-th/9110054] [INSPIRE].

[60] I. Bars and K. Sfetsos, A Superstring theory in four curved space-time dimensions, Phys. Lett. B 277 (1992) 269 [hep-th/9111040] [INSPIRE].

[61] I.T. Ivanov, B.-b. Kim and M. Roček, Complex structures, duality and WZW models in extended superspace, Phys. Lett. B 343 (1995) 133 [hep-th/9406063] [INSPIRE].

[62] K. Murata and J. Soda, A Note on separability of field equations in Myers-Perry spacetimes, Class. Quant. Grav. 25 (2008) 035006 [arXiv:0710.0221] [INSPIRE]. 